\title{
Nonlinear Waves in Solid Continua with Finite Deformation
}

\author{
K. S. Surana ${ }^{1}$, J. Knight ${ }^{1}$, J. N. Reddy ${ }^{2}$ \\ ${ }^{1}$ Department of Mechanical Engineering, University of Kansas, Lawrence, KS, USA \\ ${ }^{2}$ Department of Mechanical Engineering, Texas A \& M University, College Station, TX, USA \\ Email: kssurana@ku.edu, jknight@ku.edu, inreddy@tamu.edu
}

Received 28 July 2015; accepted 7 September 2015; published 11 September 2015

Copyright (C) 2015 by authors and Scientific Research Publishing Inc.

This work is licensed under the Creative Commons Attribution International License (CC BY). http://creativecommons.org/licenses/by/4.0/

(c) (i) Open Access

\section{Abstract}

This work considers initiation of nonlinear waves, their propagation, reflection, and their interactions in thermoelastic solids and thermoviscoelastic solids with and without memory. The conservation and balance laws constituting the mathematical models as well as the constitutive theories are derived for finite deformation and finite strain using second Piola-Kirchoff stress tensor and Green's strain tensor and their material derivatives [1]. Fourier heat conduction law with constant conductivity is used as the constitutive theory for heat vector. Numerical studies are performed using space-time variationally consistent finite element formulations derived using space-time residual functionals and the non-linear equations resulting from the first variation of the residual functional are solved using Newton's Linear Method with line search. Space-time local approximations are considered in higher order scalar product spaces that permit desired order of global differentiability in space and time. Computed results for non-linear wave propagation, reflection, and interaction are compared with linear wave propagation to demonstrate significant differences between the two, the importance of the nonlinear wave propagation over linear wave propagation as well as to illustrate the meritorious features of the mathematical models and the space-time variationally consistent space-time finite element process with time marching in obtaining the numerical solutions of the evolutions.

\section{Keywords}

Linear and Nonlinear Waves, Second Piola-Kirchoff Stress, Green's Strain, Constitutive Theories, Dissipation, Memory, Rheology, Finite Strain

\section{Literature Review, Outline, and Significance}

The subject of nonlinear wave propagation in which nonlinearity primarily arises due to consideration of finite 
deformation and finite strain is an area of significant interest due to the introduction of polymeric solids and their abundant use in industrial applications. Polymers can undergo finite deformation, finite strain, have a dissipation mechanism, and exhibit rheological behavior. Thus, the deformation physics in such materials is quite complex. Development of mathematical models for finite deformation and finite strain for solid continua in Lagrangian description using conservation and balance laws resulting in initial value problems (IVP) and time accurate numerical simulations of the evolution described by the IVPs is the main objective of this research.

A review of the published works related to the research presented here is given in the following. In the published works cited and discussed here we address four basic questions: 1) what is the source of nonlinearity, 2) type of material considered (elastic, viscoelastic, etc.), 3) constitutive theories, 4) methodology or approach used to obtain numerical solution of the resulting mathematical model. In reference [2] conservation and balance laws are considered and some aspects of the constitutive theories are also discussed with the main objective of obtaining simplified mathematical models with various assumptions that would permit theoretical or semianalytical solutions. Many specialized forms of the 1D and 2D wave equations and their possible solutions are discussed. Reference [3] considers solids under high-pressure shock compression. This book presents many aspects of mechanics, physics, and chemistry in such deformation. Plasticity or irreversible deformation processes are a central point of focus in this reference. The material in the book is largely devoted to experiments, design of experiments, and analysis of experimental data. Experimentally focused work on "nonlinear phenomena in the propagation of elastic waves in solids" is also presented in reference [4]. The authors consider Green's strain and many applications to different and unique materials. Precise mathematical models used, the constitutive theories considered and their derivations are not given. In reference [5], the authors consider a one degree of freedom oscillator subjected to an external force and a restoring viscoelastic force with memory based on a phenomenological approach. Such models are not valid in the thermodynamic sense and their extension to $\mathrm{R}^{2}$ and $\mathrm{R}^{3}$ is not possible [1]. Finite amplitude waves in isotropic elastic plates are considered by Lima and Hamilton [6]. A perturbation technique with semianalytical solution is used to obtain the solutions of the governing equation of equilibrium in Lagrangian description. Periodic harmonic solutions are presented. In reference [7], thermoelastic small-amplitude wave propagation in nonlinear elastic media is considered. Helmholtz free energy density is expressed as a nonlinear function of the principal stretches and is used to derive the constitutive equation for stress. For thermoelastic material based on reference [1], this approach of deriving constitutive theory is unfounded. This approach is applied to layered structures. Lima and Hamilton [8] presented a study of finite amplitude waves in isotropic elastic waveguides with arbitrary cross-sectional area using perturbation and modal analysis techniques to obtain the solutions of nonlinear equations of motion for harmonic motion. The second Piola-Kirchoff stress tensor is expressed as a quadratic function of the Green's strain tensor using a special form given in references [9] [10]. A study of nonlinear deformation waves in solids and dispersion due to microstructures using Mindlin type model is considered in reference [11]. Finite volume method is used to study propagation and interaction of one-dimensional waves. Nonlinear transient thermal stresses and elastic wave propagation studies in thick temperature-gradient dependent FGM cylinder using a second-order point-collocation method are presented in reference [12]. In reference [13], numerical simulations of linear and nonlinear waves in hypoelastic solids are presented using conservation element and solution element method (CESE). These investigations are hypothetical as the constitutive theories for hypoelastic solids are hypothetical since these constitutive theories cannot describe the constitution of solids. Numerical simulations of nonlinear elastic wave propagation in piecewise homogeneous media are considered in reference [14]. Wave reflection, transmission, and interaction of waves are not clearly demonstrated primarily due to complexity of the properties of the domain. Vibrations and wave propagation in thick FGM cylinders with temperature dependent material properties are investigated in reference [15]. A nodal discontinuous Galerkin finite element method is considered for nonlinear elastic wave propagation in reference [16]. Nonlinear transient stress wave propagation in thick FGM cylinder using a unified generalized thermoelasticity theory is considered in reference [17]. Nonlinear constitutive model for axisymmetric bending of annular graphene-like nanoplates with gradient elasticity enhancement effects is considered in reference [18]. In reference [19], nonlinear semianalytical finite-element algorithm for the analysis of internal resonance conditions in complex wave guides is considered. Linear stress waves in elastic medium for infinitesimal deformation linear elasticity have been studied by Surana et al. [20].

From the brief literature review presented here we note the following. 1) The mathematical models resulting from conservation and balance laws are not explicitly defined and stated in most cases. 2) The constitutive theo- 
ries for thermoelastic and thermoviscoelastic materials with and without memory and the basis for their derivations are mostly absent. In many instances phenomenological approach is used. 3) A mix of various space-time decoupled methods based on finite volume, finite element approaches for discretization in space followed by some time integration scheme is used to obtain evolutions described by the IVPs. In many instances semi-analytical approaches are considered for highly simplified mathematical models that lack the desired physics. 4) In the model problems considered and the numerical studies presented for them, the complexity of the physics of the model problem rarely permits the assessment of the importance of nonlinearity when compared to the corresponding solutions from the linear models. 5) The issue of time accuracy of numerical solutions is never addressed in any of the references. This is of utmost significance as only with the correct time evolution can we assess the importance and significance of the nonlinear wave propagation.

The outline of the work presented in the paper is given in the following. General considerations and scope of study are contained in Section 2. The mathematical models in $\mathrm{R}^{3}$ (3D) are presented in Section 3. The mathematical models in $R^{1}$ (1D) are given in Section 4 . The dimensionless forms of the mathematical models in $R^{1}$ are presented in Section 4.4. The computational framework, the space-time finite element formulations based on space-time residual functional and the time marching procedure for computing evolutions are presented in Section 5. Descriptions of model problems, schematics, loadings, boundary conditions and the material coefficients are given in Sections 6, 6.1, and 6.2. Computations of evolutions, convergence of the solutions, converged numerical results for the three types of solid continua considered here are presented in Section 6.3. Summary of the work and the conclusions drawn from the work presented in this paper are given in Section 7.

There are many important and meritorious aspects of the present work compared to the published works. Conservation and balance laws including constitutive theories are presented in $\mathrm{R}^{3}$ and $\mathrm{R}^{1}$ for finite deformation and finite strain in Lagrangian description for thermoelastic and thermoviscoelastic solids with and without memory using second Piola-Kirchoff stress tensor and Green's strain tensor as conjugate pair. Dimensionless forms of the mathematical models in $\mathrm{R}^{1}$ are used for presenting linear as well as nonlinear wave initiation, propagation, reflection and subsequent propagation. Some of the significant aspects of the present work are: 1) demonstration of the fact that nonlinear waves result in shock formation and complex thermal field due to dissipation. 2) Wave amplitude decay and base elongation due to dissipation are clearly demonstrated for linear as well as nonlinear waves in case of solid continua with damping. 3) Rheological behavior due to memory is demonstrated for thermoviscoelastic solid continua with memory. 4) The finite element formulations used based on space-time couple approach are shown to be free of inherent numerical dispersion. 5) Computations of evolutions for large values of time are presented to illustrate various aspects of linear and nonlinear wave propagation. 6) It is clearly shown that extremely low values of space-time residuals obtained for all numerical computations confirm time accuracy (i.e. proximity of the computed solutions to the theoretical solutions) of the results presented in the paper.

\section{Considerations in the Present Study and the Scope of Work}

The work presented here considers nonlinear wave propagation, reflection and interaction in thermoelastic solid continua and thermoviscoelastic solid continua with and without memory. The mathematical models in Lagrangian description consist of conservation and balance laws and the appropriate constitutive theories for stress tensor and heat vector [1]. The primary source of nonlinearity is due to finite deformation and finite strain. The contravariant second Piola-Kirchoff stress tensor and Green's strain are used as conjugate pairs in the derivations of the balance laws and the constitutive theories. The solid continua are assumed compressible thus permitting finite deformation and associated changes in density. For thermoelastic solid, rate constitutive theory of order zero is used in which the contravariant second Piola-Kirchoff stress is a linear function of the Green's strain tensor. The work presented here only considers thermal affects due to rate of entropy production associated with rate of dissipation due to rate of mechanical work, thus for thermoelastic solids, the energy equation and entropy inequality resulting from the first and second law of thermodynamics are not required. In the case of thermoviscoelastic solids with and without memory, the second Piola-Kirchoff stress tensor is decomposed into equilibrium stress tensor and deviatoric stress tensor. The constitutive theory for the second Piola-Kirchoff equilibrium stress tensor is derived in terms of thermodynamic pressure. The constitutive theory for deviatoric second Piola-Kirchoff stress tensor for thermoviscoelastic solids without memory is considered as a first order rate theory [1] in which the deviatoric second Piola-Kirchoff stress tensor is a linear function of the Green's 
strain tensor and its material derivative. In the case of thermoviscoelastic solids with memory, the constitutive theory for deviatoric second Piola-Kirchoff stress tensor is a first order rate theory in deviatoric second PiolaKirchoff stress tensor as well as Green's strain tensor.

The mathematical models are non-dimensionalized for use in the computational framework. Explicit forms of the mathematical models are presented in $\mathrm{R}^{1}$. These models are used to study one dimensional nonlinear wave propagation, reflection, and interaction in the three types of solid continua considered here. Linear forms of these mathematical models based on small-strain small-deformation assumptions are also considered in the numerical studies. The evolutions of the nonlinear and linear waves are compared to demonstrate the differences between the two. Ramp and pulse stress loadings and pulse velocity loading are considered in the numerical studies.

The dimensionless form of the mathematical models in $\mathrm{R}^{1}$ are utilized to construct the space-time coupled finite element processes for an increment of time (giving a space-time strip) based on space-time residual functionals that are space-time variationally consistent, hence the computations during the entire evolution remain unconditionally stable. Evolutions are computed by time marching using the space-time strip. The space-time local approximations for the dependent variables over a space-time element are considered in higher order scalar product spaces that permit higher order global differentiability of the space-time approximations over a discretization of the strip as well as at the inter-strip boundaries. The minimally conforming spaces ensure the spacetime integrals over discretization of a space-time strip are in the Riemann sense. This feature enables computations of time accurate evolutions.

\section{Mathematical Models in $\mathbf{R}^{3}$}

In this section we present mathematical models for thermoelastic and thermoviscoelastic solids with and without memory consisting of conservation and balance laws and the constitutive theories. The mathematical models are first presented in $\mathrm{R}^{3}$. These are then followed by explicit forms of the mathematical models in $\mathrm{R}^{1}$ for 1-D wave propagation including their dimensionless forms. Finite deformation and finite strain are considered in the mathematical models. Contravariant second Piola-Kirchoff stress and Green's strain tensor are used as conjugate pairs [1]. Solid continua are considered compressible. In the mathematical models, the energy equation is only considered if the rate of mechanical work results in entropy production. The mathematical models are considered in Lagrangian description.

\subsection{Thermoelastic Solid Continua in $\mathbf{R}^{3}$}

In such solid continua the deformation process is reversible; hence rate of mechanical work does not result in rate of entropy production. Thus, the specific internal energy in the absence of strain energy is not affected by the rate of work. As a consequence, mechanical deformation and thermal effects remain uncoupled; hence the thermal behavior can be studied independent of the mechanical deformation. Since in the present work we only consider thermal effects due to rate of entropy production resulting from the rate of work, for thermoelastic solids the mathematical model only consists of conservation of mass, balance of linear momenta, and balance of angular momenta. The energy equation in this case is a linear (or nonlinear) diffusion equation and entropy inequality contains no dissipation terms but forms the basis for deriving constitutive theory for the heat vector appearing in the energy equation. The constitutive theory for the contravariant second Piola-Kirchoff stress $\left(\sigma^{[0]}\right)$ is based on $\sigma^{[0]}$ and Green's strain tensor $\left(\varepsilon_{[0]}\right)$ as conjugagte pair and is derived using strain energy density function or theory of generators and invariants (see reference [1] for details). Thus for compressible thermoelastic solids, the mathematical model consists of continuity equation (conservation of mass), momentum equations (balance of linear momenta), balance of angular momenta, and the constitutive theory for the stress tensor. In the absence of body forces, we can have the following in Lagrangian description [1]. In the constitutive equation for $\sigma^{[0]}$ we assume $\sigma^{[0]}$ as a linear function of $\varepsilon_{[0]}$.

$$
\begin{gathered}
\rho_{0}=|J| \rho(\boldsymbol{x}, t) ; \quad \text { continuity equation } \\
\rho_{0}\{\dot{v}\}-\left([J]\left[\sigma^{[0]}\right]^{\mathrm{T}}\right)\{\nabla\}=0 ; \quad \text { momentum equation }
\end{gathered}
$$




$$
\begin{gathered}
{\left[\sigma^{[0]}\right]=\left[\sigma^{[0]}\right]^{\mathrm{T}} ; \quad \text { balance of angular momenta }} \\
{\left[\sigma^{[0]}\right]=2 \mu\left[\varepsilon_{[0]}\right]+\lambda\left(\operatorname{tr}\left[\varepsilon_{[0]}\right]\right)[I]} \\
\text { or } \\
\left\{\sigma^{[0]}\right\}=[D]\left\{\varepsilon_{[0]}\right\}
\end{gathered}
$$

In which

$$
\begin{aligned}
& {[J]=\left[\frac{\partial\{\bar{x}\}}{\partial\{x\}}\right]=\left[\left[\frac{\partial\{u\}}{\partial\{x\}}\right]+[I]\right]} \\
& \{v\}=\{\dot{u}\} \\
& \left\{\sigma^{[0]}\right\}^{\mathrm{T}}=\left[\sigma_{11}^{[0]} \sigma_{22}^{[0]} \sigma_{33}^{[0]} \sigma_{23}^{[0]} \sigma_{31}^{[0]} \sigma_{12}^{[0]}\right] \\
& \left\{\varepsilon_{[0]}\right\}^{\mathrm{T}}=\left[\left(\varepsilon_{[0]}\right)_{11}\left(\varepsilon_{[0]}\right)_{22}\left(\varepsilon_{[0]}\right)_{33}\left(\varepsilon_{[0]}\right)_{23}\left(\varepsilon_{[0]}\right)_{31}\left(\varepsilon_{[0]}\right)_{12}\right] \\
& {\left[\varepsilon_{[0]}\right]=\frac{1}{2}\left([J]^{\mathrm{T}}[J]-[I]\right)} \\
& D_{i i}=2 \mu+\lambda ; D_{i j}=\lambda, i \neq j ; D_{i i}=2 \mu, i=4,5,6 \\
& D_{i j}=0 ; i, j=4,5,6 \text { and } i \neq j
\end{aligned}
$$

The $\bar{x}_{i}=x_{i}+u_{i}$ are coordinates of a material point $P(x, t)$ in the current configuration, $u_{i}$ are displacements in the $x_{i}$ directions and $v_{i}$ are the corresponding velocities. The density in the reference configuration $(t=0)$ is given by $\rho_{0}$ and $\rho(x, t)$ is the density of the material point $P(x, t)$ in the current configuration at time $t$. Subscripts 1,2 , and 3 in (3.7) refer to $x_{1}, x_{2}$, and $x_{3}$ axes of a fixed $x$-frame. A dot (.) on the quantity implies material derivative. Equation (3.6) can be substituted into (3.2) thereby eliminating $v$ as a dependent variable. The contravariant second Piola-Kirchoff stress tensor is symmetric (3.3). Thus the mathematical model reduces to

$$
\begin{gathered}
\rho_{0}=|J| \rho(\boldsymbol{x}, \boldsymbol{t}) \\
\rho_{0}\{\ddot{u}\}-\left([J]\left[\sigma^{[0]}\right]^{\mathrm{T}}\right)\{\nabla\}=0 \\
\left\{\sigma^{[0]}\right\}=[D]\left\{\varepsilon_{[0]}\right\}
\end{gathered}
$$

in which $\left[\varepsilon_{[0]}\right]$ and $[D]$ are defined by (3.8) and (3.9). Material coefficients are $\lambda$ and $\mu$. When the $u_{i}$, hence $[J]$, are known, the density, $\rho$ in the current configuration, is deterministic from (3.10). Thus, for thermoelastic compressible solid continua, $\rho(x, t)$ is not a dependent variable in the mathematical model. Equations (3.11) and (3.12) are nine partial differential equations in three displacements $u_{i}$ and six stresses $\sigma_{i j}^{[0]}\left(=\sigma_{j i}^{[0]}\right)$, hence the mathematical model has closure. Equations (3.10)-(3.12) and $\left[\varepsilon_{[0]}\right]$ defined by 8 is the final form of the mathematical model for thermoelastic solids in $\mathrm{R}^{3}$ in which (3.10) only needs to be used to determine $\rho(x, t)$ once $[J]$ is known in the current configuration.

\subsection{Thermoviscoelastic Solid Continua without Memory in $\mathbf{R}^{3}$}

In such solid continua the deformation process is not reversible due to rate of mechanical work resulting in entropy production (dissipation) which affects the specific internal energy. Hence, in such solid continua, the material exhibits elasticity as well as dissipation mechanism but has no memory (or rheology). In such solid continua, the mechanical deformation and thermal effects are coupled implying that the energy equation resulting from the first law of thermodynamics is an integral part of the complete mathematical model. Entropy inequality resulting from the second law of thermodynamics along with decomposition of $\sigma^{[0]}$ into equilibrium 
$\left({ }_{e} \sigma^{[0]}\right)$ and deviatoric $\left({ }_{d} \sigma^{[0]}\right)$ contravariant second Piola-Kirchoff stress tensor provides mechanism for deriving constitutive theory for heat vector and $\left({ }_{e} \sigma^{[0]}\right)$ and additionally requiress that rate of work due to $\left({ }_{d} \sigma^{[0]}\right)$ be positive. The constitutive theory for ${ }_{d} \sigma^{[0]}$ is derived using the theory of generators and invariants [1]. The complete mathematical model for thermoviscoelastic solid continua without memory consists of conservation of mass, balance of linear momenta, balance of angular momenta, which are the same as in the case of thermoelastic solids (Equations (3.1)-(3.3)). Additionally, the energy equation and constitutive theories for ${ }_{e} \boldsymbol{\sigma}^{[0]},{ }_{d} \boldsymbol{\sigma}^{[0]}$, and heat vector $\boldsymbol{q}$ are needed. The complete mathematical model is given in the following in Lagrangian description for compressible matter (in the absence of body forces). The constitutive theory used for ${ }_{d} \sigma^{[0]}$ is a simple first order linear rate theory in which ${ }_{d} \sigma^{[0]}$ is a linear function of $\varepsilon_{[0]}$ and $\dot{\varepsilon}_{[0]}$ (material derivative of $\varepsilon_{[0]}$ ). The constitutive theory for $\{q\}$ is simple Fourier heat conduction law [1]. The constitutive theory for ${ }_{e} \boldsymbol{\sigma}^{[0]}$ is in terms of thermodynamic pressure [1].

$$
\begin{aligned}
& \rho_{0}=|J| \rho(\boldsymbol{x}, t) ; \quad \text { continuity } \\
& \rho_{0}\{\ddot{u}\}-\left([J]\left[\sigma^{[0]}\right]^{\mathrm{T}}\right)\{\nabla\}=0 ; \quad \text { balance of linear momenta } \\
& {\left[\sigma^{[0]}\right]=\left[\sigma^{[0]}\right]^{\mathrm{T}} ; \quad \text { balance of angular momenta }} \\
& \rho_{0} \dot{e}+\{\nabla\}^{\mathrm{T}}\{q\}-\operatorname{tr}\left(\left[\sigma^{[0]}\right]^{\mathrm{T}}\left[\dot{\varepsilon}_{[0]}\right]^{\mathrm{T}}\right)=0 ; \quad \text { energy equation } \\
& {\left[\sigma^{[0]}\right]=\left[{ }_{e} \sigma^{[0]}\right]+\left[{ }_{d} \sigma^{[0]}\right]} \\
& {\left[e^{[0]}\right]=-p|J|[J]^{\mathrm{T}}[J]^{-1}} \\
& {\left[{ }_{d} \sigma^{[0]}\right]=2 \underset{\sim}{\mu}\left[\varepsilon_{[0]}\right]+\lambda\left(\operatorname{tr}\left[\varepsilon_{[0]}\right]\right)[I]+2 \underset{\sim}{\mu}\left[\dot{\varepsilon}_{[0]}\right]+\lambda_{\mathcal{1}}\left(\operatorname{tr}\left[\dot{\varepsilon}_{[0]}\right]\right)[I]} \\
& \text { or }\left\{{ }_{d} \sigma^{[0]}\right\}=[\underset{\sim}{D}]\left\{\varepsilon_{[0]}\right\}+[\underset{\sim}{B}]\left\{\dot{\varepsilon}_{[0]}\right\} \\
& D_{\sim i i}=2 \underset{\sim}{\mu}+\underset{\sim}{\lambda} ;{\underset{\sim}{i j}}_{i j}=\underset{\sim}{\lambda}, i \neq j ; \underset{\sim}{D_{i i}}=2 \underset{\sim}{\mu}, i, j=4,5,6 \\
& D_{i j}=0 ; i, j=4,5,6 \text { and } i \neq j \\
& {\underset{\sim}{B i}}_{B}=2 \mu_{\sim}+\lambda_{\sim} ; B_{i j}=\lambda_{1}, i \neq j ;{\underset{\sim}{i i}}_{i}=2 \mu_{\sim}, i, j=4,5,6 \\
& B_{i j}=0, i, j=4,5,6 \text { and } i \neq j
\end{aligned}
$$

and

$$
\{q\}=-k\{\nabla \theta\} ; \quad \text { Fourier heat conduction law }
$$

Here $\mu_{1}$ and $\tilde{\lambda}_{1}$ are material coefficients related to dissipation, $k$ is thermal conductivity, $\theta$ is absolute temperature, and $e$ is specific internal energy. The compressive thermodynamic pressure, $p$ in (3.17) is assumed positive. Equation of state, $p=p(\rho, \theta)$ is known for each specific solid continua under consideration.

\subsection{Thermoviscoelastic Solids with Memory in $\mathbf{R}^{3}$}

In such solid continua the deformation process is also not reversible. In these solids the rate of mechanical work also results in rate of entropy production (dissipation). Additionally, such solids exhibit rheological behavior, i.e. memory. Due to rate of entropy production, the thermal and mechanical effects are coupled; hence the energy equation is an integral part of the complete mathematical model. Entropy inequality resulting from the second law of thermodynamics along with the stress decomposition $\sigma^{[0]}={ }_{e} \sigma^{[0]}+{ }_{d} \sigma^{[0]}$ provides mechanism for deriving constitutive theories for ${ }_{e} \sigma^{[0]}$ and heat vector and additionally requires that rate of work due to ${ }_{d} \sigma^{[0]}$ be positive. The constitutive theory for ${ }_{d} \sigma^{[0]}$ is derived using theory of generators and invariants [1]. The complete mathematical model for thermoviscoelastic solids with memory, in Lagrangian description, consists of continuity equation, momentum equations, energy equation, and constitutive theories for ${ }_{e} \boldsymbol{\sigma}^{[0]},{ }_{d} \boldsymbol{\sigma}^{[0]}$, and $\boldsymbol{q}$. Constitutive theories used here are first order linear rate theories in ${ }_{d} \sigma^{[0]}$ and $\varepsilon_{[0]}$, i.e. material derivative of 
${ }_{d} \sigma^{[0]}$ is a linear function of $\varepsilon_{[0]}, \quad \dot{\varepsilon}_{[0]}$, and $\sigma^{[0]}$. This constitutive theory permits dissipation as well as rheology. Constitutive theory used for $\boldsymbol{q}$ is a simple Fourier heat conduction law. The constitutive theory for ${ }_{e} \boldsymbol{\sigma}^{[0]}$ is in terms of thermodynamic pressure $p(\rho, \theta)$. The complete mathematical model is given in the following (in the absence of body forces).

$$
\begin{gathered}
\rho_{0}=|J| \rho(\boldsymbol{x}, t) ; \quad \text { continuity } \\
\rho_{0}\{\ddot{u}\}-\left([J]\left[\sigma^{[0]}\right]^{\mathrm{T}}\right)\{\nabla\}=0 ; \quad \text { balance of linear momenta } \\
{\left[\sigma^{[0]}\right]=\left[\sigma^{[0]}\right]^{\mathrm{T}} ; \quad \text { balance of angular momenta }} \\
\rho_{0} \dot{e}+\{\nabla\}^{\mathrm{T}}\{q\}-\operatorname{tr}\left(\left[\sigma^{[0]}\right]^{\mathrm{T}}\left[\dot{\varepsilon}_{[0]}\right]^{\mathrm{T}}\right)=0 ; \quad \text { energy equation } \\
{\left[\sigma^{[0]}\right]=\left[{ }_{e} \sigma^{[0]}\right]+\left[{ }_{d} \sigma^{[0]}\right]} \\
{\left[{ }_{e} \sigma^{[0]}\right]=-p|J|[J]^{\mathrm{T}}[J]^{-1}} \\
{\left[{ }_{d} \sigma^{[1]}\right]+c_{1}\left[{ }_{d} \sigma^{[0]}\right]+c_{2}\left(\operatorname{tr}\left[{ }_{d} \sigma^{[0]}\right]\right)[I]} \\
=a_{0}^{1}\left[\varepsilon_{[0]}\right]+a_{0}^{2}\left(\operatorname{tr}\left[\varepsilon_{[0]}\right]\right)[I]+a_{1}^{1}\left[\varepsilon_{[1]}\right]+a_{1}^{2}\left(\operatorname{tr}\left[\varepsilon_{[1]}\right]\right)[I] \\
\text { or }\left\{{ }_{d} \sigma^{[1]}\right\}+[c]\left\{{ }_{d} \sigma^{[0]}\right\}=\left[a_{0}\right]\left\{\varepsilon_{[0]}\right\}+\left[a_{1}\right]\left\{\varepsilon_{[1]}\right\}
\end{gathered}
$$

where coefficients of $[\underset{\sim}{c}],\left[\mathfrak{a}_{0}\right]$, and $\left[a_{1}\right]$ are functions of $\left(c_{1}, c_{2}\right),\left(a_{0}^{1}, a_{0}^{2}\right)$, and $\left(a_{1}^{1}, a_{1}^{2}\right)$ and are defined in the same manner as coefficients of $[D]$ in (3.18). Additionally, $\boldsymbol{q}$ is defined as

$$
\{q\}=-k\{\nabla \theta\} ; \quad \text { Fourier heat conduction law }
$$

We consider compressive thermodynamic pressure to be positive, hence the negative sign in the constitutive theory for ${ }_{e} \sigma^{[0]}$. Here also, $p=p(\rho, \theta)$ is an equation of state and is known for a material under consideration. The constitutive theory for ${ }_{d} \sigma^{[0]}$ (last equation in (3.24)) can also be written (similar to thermoviscoelastic solid continua without memory) in the following form if we neglect $\operatorname{tr}\left(\left[{ }_{d} \sigma^{[0]}\right]\right)$ in equation three in (3.24), divide throughout by $c_{1}$, and define $\frac{1}{c_{1}}$ as $\lambda$.

$$
\left\{{ }_{d} \sigma^{[0]}\right\}+\lambda_{1}\left\{{ }_{d} \sigma^{[1]}\right\}=\left[\underline{a}_{0}\right]\left\{\varepsilon_{[0]}\right\}+\left[\underline{a}^{1}\right]\left\{\varepsilon_{[1]}\right\}
$$

in which

$$
\left[\underline{a}_{0}\right]=\frac{1}{c_{1}}\left[\underline{a}_{0}\right],\left[\underline{a}_{1}\right]=\frac{1}{c_{1}}\left\{\varepsilon_{[1]}\right\}
$$

Equation (3.26) is the final form used in the present work to obtain its equivalent form in $\mathrm{R}^{1}$.

\section{Remarks:}

Even though the model problems considered in the present work are wave propagation studies in $\mathrm{R}^{1}$, the mathematical models in $\mathrm{R}^{3}$ are necessary to demonstrate the presence of all relevant terms, many of which drop out in $\mathrm{R}^{1}$ as in $\mathrm{R}^{1}$ there is no concept of the other two dimensions.

\section{Mathematical Models in $\mathrm{R}^{1}$}

In this section explicit forms of the mathematical models for $1 \mathrm{D}$ wave propagation in $\mathrm{R}^{1}$ for thermoelastic and thermoviscoelastic solid continua with and without memory are presented. These models are derived using the mathematical models presented in Section 3 for the three dimensional case, i.e. in $\mathrm{R}^{3}$, hence they hold for finite deformation and finite strain. We assume directions 1 and $x_{1}$ to be the same as $x$. Displacement $u_{1}$ in $x_{1}$ (or $x$ ) direction is denoted by $u$ and the velocity $v_{1}$ by $v$. Details of the mathematical models based on conservation and balance laws and the constitutive theories for the three types of material behaviors considered 
are given in the following (in the absence of body forces).

\subsection{Thermoelastic Solid Matter (Compressible): $\mathbf{R}^{1}$}

For 1D wave propagation in $\mathrm{R}^{1}$ the mathematical models of Section 3.1 in $\mathrm{R}^{3}$ reduce to

$$
\begin{aligned}
& \rho_{0}=\left(f \frac{\partial u}{\partial x}+1\right) \rho(x, t) \\
& \rho_{0} \frac{\partial^{2} u}{\partial t^{2}}-\frac{\partial}{\partial x}\left(\left(f \frac{\partial u}{\partial x}+1\right) \sigma_{x x}^{[0]}\right)=0 \\
& \sigma_{x x}^{[0]}=E\left(\frac{\partial u}{\partial x}+f \frac{1}{2}\left(\frac{\partial u}{\partial x}\right)^{2}\right)
\end{aligned}
$$

in which $f=0$ for small deformation and small strain and $f=1$ for finite deformation and finite strain. This is a mathematical model in dependent variable $u$ and $\sigma_{x x}^{[0]}$. E is material coefficient in the reference configuration.

Alternate form of the mathematical model using $v$

It is some times more convenient to introduce velocity $v=\frac{\partial u}{\partial t}$ as a dependent variable in the mathematical model. This form of the mathematical model is specially helpful in studies in which velocity needs to be specified as a boundary condition or initial condition. Thus, using velocity $v=\frac{\partial u}{\partial t}$ as a dependent variable Equation (4.1) becomes

$$
\begin{aligned}
& \rho_{0}=\left(f \frac{\partial u}{\partial x}+1\right) \rho(x, t) \\
& \rho_{0} \frac{\partial v}{\partial t}-\frac{\partial}{\partial x}\left(\left(f \frac{\partial u}{\partial x}+1\right) \sigma_{x x}^{[0]}\right)=0 \\
& \sigma_{x x}^{[0]}=E\left(\frac{\partial u}{\partial x}+f \frac{1}{2}\left(\frac{\partial u}{\partial x}\right)^{2}\right) \\
& v=\frac{\partial u}{\partial t}
\end{aligned}
$$

This mathematical model contains dependent variables $u, \sigma_{x x}^{[0]}$, and $v$.

\subsection{Thermoviscoelastic Solids without Memory in $\mathbf{R}^{1}$}

Using equations in Section 3.2, we can obtain the following in $\mathrm{R}^{1}$. We consider $\dot{e}=c_{p 0} \dot{\theta}=c_{p 0} \frac{\partial \theta}{\partial t}$.

$$
\begin{aligned}
& \rho_{0}=\left(f \frac{\partial u}{\partial x}+1\right) \rho(x, t) \\
& \rho_{0} \frac{\partial^{2} u}{\partial t^{2}}-\frac{\partial}{\partial x}\left(\left(f \frac{\partial u}{\partial x}+1\right)\left(e_{e} \sigma_{x x}^{[0]}+{ }_{d} \sigma_{x x}^{[0]}\right)\right)=0 \\
& { }_{e} \sigma_{x x}^{[0]}=-p\left(f \frac{\partial u}{\partial x}+1\right) \\
& { }_{d} \sigma_{x x}^{[0]}={ }^{d} \tilde{E}\left(\varepsilon_{[0]}\right)+{ }_{x x}+{ }^{d} \mathcal{\sim}\left(\dot{\varepsilon}_{[0]}\right) \\
& \left.\left(\varepsilon_{x x}\right)=\frac{\partial u}{\partial x}+f \frac{1}{2}\left(\frac{\partial u}{\partial x}\right)^{2}\right)_{x x} \\
& \rho_{0} c_{p 0} \frac{\partial \theta}{\partial t}+\frac{\partial q}{\partial x}-{ }_{d} \sigma_{x x}^{[0]}\left(\dot{\varepsilon}_{[0]}\right)=0 \\
& q=-k \frac{\partial \theta}{\partial x}
\end{aligned}
$$

in which $p(\rho, \theta)$ is thermodynamic pressure defined by the equation of state, thus ${ }_{e} \sigma_{x x}^{[0]}$ is defined in terms 
of known $p(\rho, \theta)$ in (4.3). Equations (4.3) are five partial differential equations in $u,{ }_{d} \sigma_{x x}^{[0]},\left(\varepsilon_{[0]}\right)_{x x}, q$, and $\theta$, hence the mathematical model has closure. Material coefficients ${ }^{d} E$ and ${ }^{d} C$ are related to elasticity and dissipation respectively and are defined in the reference configuration.

\section{Remarks}

For solid matter the equation of state is rather involved [1] even though there is no particular problem in incorporating it in (4.3). Since the main objective of this research is the study of linear and nonlinear wave propagation, the constitutive theory for ${ }_{e} \sigma_{x x}^{[0]}$ is modified by considering the solid continua to be incompressible just for the purposes of establishing the constitutive theory for the equilibrium stress ${ }_{e} \sigma_{x x}^{[0]}$. The same assumption is applied to linear and nonlinear wave propagation so that the comparisons of linear and nonlinear wave propagation studies remain meaningful. This is obviously an assumption that will undoubtedly influence the model behavior, the extent of which is believed to be not serious. There is further work in progress that incorporates actual equations of state for $p(\rho, \theta)$ for compressible solid matter. This work is expected to provide quantitative measures of the deviations in true behavior of wave propagation due to incompressibility assumption for the constitutive theory for equilibrium stress. For incompressible matter, equilibrium stress is mean normal stress. Following [1], for incompressible solid matter we have the following in $\mathrm{R}^{1}$

$$
{ }_{e} \sigma_{x x}^{[0]}=\frac{1}{2}{ }_{d} \sigma_{x x}^{[0]} ; \quad \sigma_{x x}^{[0]}=\frac{3}{2}{ }_{d} \sigma_{x x}^{[0]} ; \quad{ }_{d} \sigma_{x x}^{[0]}=\frac{2}{3} \sigma_{x x}^{[0]}
$$

Using (4.4), ${ }_{e} \sigma_{x x}^{[0]}$ in (4.3) can be expressed either in terms of ${ }_{d} \sigma_{x x}^{[0]}$ or $\sigma_{x x}^{[0]}$ and the resulting mathematical model can likewise be expressed either in terms of $\sigma_{x x}^{[0]}$ or ${ }_{d} \sigma_{x x}^{[0]}$. In the following, we choose $\sigma_{x x}^{[0]}$ so that this mathematical model contains the same stress measure as in case of thermoelastic solids 4.1.

$$
\begin{aligned}
& \rho_{0}=\left(f \frac{\partial u}{\partial x}+1\right) \rho(x, t) \\
& \rho_{0} \frac{\partial^{2} u}{\partial t^{2}}-\frac{\partial}{\partial x}\left(\left(f \frac{\partial u}{\partial x}+1\right) \sigma_{x x}^{[0]}\right)=0 \\
& \sigma_{x x}^{[0]}=E\left(\varepsilon_{[0]}\right)_{x x}+c\left(\dot{\varepsilon}_{[0]}\right)_{x x} ; E=\frac{3}{2}{ }^{d} \underset{\sim}{E}, c=\frac{3}{2}^{d}{ }_{\sim}^{C} \\
& \left(\varepsilon_{[0]}\right)_{x x}=\frac{\partial u}{\partial x}+f \frac{1}{2}\left(\frac{\partial u}{\partial x}\right)^{2} \\
& \rho_{o} c_{p 0} \frac{\partial \theta}{\partial t}+\frac{\partial q}{\partial x}-\frac{2}{3} \sigma_{x x}^{[0]}\left(\dot{\varepsilon}_{[0]}\right)_{x x}=0 \\
& q=-k \frac{\partial \theta}{\partial x}
\end{aligned}
$$

The factor of $\frac{2}{3}$ in the dissipation term, in the energy equation, is due to incompressibility assumption in the constitutive theory. Here also $f=0$ for small deformation and small strain and $f=1$ for finite deformation and finite strain. Absolute temperature is given by $\theta$. Thus, we have five partial differential equations (not including continuity) in five dependent variables $u, \sigma_{x x}^{[0]},\left(\varepsilon_{[0]}\right)_{x x}, q$, and $\theta$, thus the mathematical model has closure. Material coefficients $E$ and $c$ define the modulus of elasticity and dissipation coefficient respectively. An alternate form of (4.5) can be derived by using $v=\frac{\partial u}{\partial t}$ as additional equation in (4.5) and by replacing $\frac{\partial^{2} u}{\partial t^{2}}$ in the second equation in (4.5) by $\frac{\partial v}{\partial t}$. This model contains $v$ as an additional variable (compared to (4.5)) but also contains additional equation $v=\frac{\partial u}{\partial t}$, hence has closure.

\subsection{Thermoviscoelastic Solids with Memory in $\mathrm{R}^{1}$}

Using the mathematical model of Section 3.3 (in $\mathrm{R}^{3}$ ) we can obtain the explicit form of the equations in the mathematical model in $\mathrm{R}^{1}$. In this case also we employ Equation (4.4). The final form of the equations for the mathematical model in $\mathrm{R}^{1}$ is given in the following (in terms of $\sigma_{x x}^{[0]}$ ). 


$$
\begin{aligned}
& \rho_{0}=\left(f \frac{\partial u}{\partial x}+1\right) \rho(x, t) \\
& \rho_{0} \frac{\partial^{2} u}{\partial t^{2}}-\frac{\partial}{\partial x}\left(\left(f \frac{\partial u}{\partial x}+1\right) \sigma_{x x}^{[0]}\right)=0 \\
& \sigma_{x x}^{[0]}+\lambda \frac{\partial \sigma_{x x}^{[0]}}{\partial t}=E\left(\varepsilon_{[0]}\right)_{x x}+c\left(\dot{\varepsilon}_{[0]}\right)_{x x} ; E=\frac{3}{2}^{d}{\underset{\sim}{E}, c=\frac{3}{2}^{d}{ }_{\sim}^{C}}_{\sim} \\
& \left(\varepsilon_{[0]}\right)_{x x}=\frac{\partial u}{\partial x}+f \frac{1}{2}\left(\frac{\partial u}{\partial x}\right)^{2} \\
& \rho_{o} c_{p 0} \frac{\partial \theta}{\partial t}+\frac{\partial q}{\partial x}-\frac{2}{3} \sigma_{x x}^{[0]}\left(\dot{\varepsilon}_{[0]}\right)_{x x}=0 \\
& q=-k \frac{\partial \theta}{\partial x}
\end{aligned}
$$

Here $E=\frac{3}{2}{ }_{\sim}^{d} \underset{\sim}{e}$ and $c=\frac{3}{2}{ }^{d} \underset{\sim}{c}$ are elastic and dissipation material coefficients and $\lambda$ is relaxation time. This model has five equations and five dependent variables (same as for thermoviscoelastic solids without memory). Similar to Section 4.2, here also, we can derive an alternate form of (4.6) by using velocity $v$ as a dependent variable. Here also $f=0$ for small deformation and $f=1$ for finite deformation. The factor of $\frac{2}{3}$ in the energy equation is due to incompressibility assumption in the constitutive theory.

\subsection{Dimensionless Form of the Mathematical Models in $\mathbf{R}^{1}$}

We present the dimensionless forms of the mathematical models given in Sections 4.1-4.3 by choosing appropriate reference quantities. We consider the mathematical models derived in Sections 4.1-4.3 and introduce hat $(\hat{)}$ i.e. $x$ changes to $\hat{x}, t$ to $\hat{t}, \theta$ to $\hat{\theta}$, etc. This implies that all quantities with hat have their usual dimensions or units in terms of force $(\hat{F})$, length $(\hat{\hat{L}})$, and time $(\hat{t})$. Next we choose a reference value of force $\left(F_{0}\right)$, length $\left(L_{0}\right)$, and time $\left(t_{0}\right)$ which would yield dimensionless force $(F)$, length $(L)$, and time $(t)$, the quantities without hat $(\hat{)})$, as $F=\frac{\hat{F}}{F_{0}}, L=\frac{\hat{L}}{L_{0}}$, and $t=\frac{\hat{t}}{t_{0}}$. This is a general process of nondimensionalizing. Additionally, we may have to choose other reference quantities too, for example, $\theta_{0}$ for temperature $\hat{\theta}$ so that we can obtain dimensionless temperature $\theta=\frac{\hat{\theta}}{\theta_{0}}$. For wave propagation the reference speed of sound is a good choice for reference velocity $\left(v_{0}\right)$. If we choose $L_{0}$ as reference length then with $v_{0}$ and $L_{0}$, reference time $t_{0}=\frac{L_{0}}{v_{0}}$, i.e. $t_{0}$ cannot be independent of $L_{0}$ and $v_{0}$. We consider the following reference quantities, the resulting dimensionless variables, and the dimensionless parameters.

$$
\begin{aligned}
& x=\frac{\hat{x}}{L_{0}}, \quad \rho_{0}=\frac{\hat{\rho}}{\tilde{\rho}_{0}}, \quad c_{p 0}=\frac{\hat{c}_{p}}{\tilde{c}_{p 0}}, \quad u=\frac{\hat{u}}{L_{0}}, \quad k=\frac{\hat{k}}{k_{0}} \\
& { }_{d} \sigma_{x x}^{[0]}=\frac{{ }_{d} \hat{\sigma}_{x x}^{[0]}}{\tau_{0}}, \quad \sigma_{e x}^{[0]}=\frac{{ }^{e_{x x}}}{\tau_{0 x}}, \quad \sigma_{x x}^{[0]}=\frac{\hat{\sigma}_{x x}^{[0]}}{\tau_{0}}, \\
& E=\frac{\hat{E}}{E_{0}}, \quad v_{0}=\sqrt{\frac{E_{0}}{\tilde{\rho}_{0}}} \quad \text { (reference speed of sound) } \\
& \tau_{0}=E_{0}=\tilde{\rho}_{0} v_{0}^{2} \quad \text { (characteristic kinetic energy) } \\
& t_{0}=\frac{L_{0}}{v_{0}}, \quad \theta=\frac{\hat{\theta}}{\theta_{0}} \\
& \tilde{\rho}_{0} \text { and } \quad \tilde{c}_{p 0} \text { are reference values of density and specific heat }
\end{aligned}
$$


Using (4.7), the mathematical models in Sections 4.1-4.3 can be nondimensionalized.

\subsubsection{Thermoelastic Solids: $\mathbf{R}^{1}$}

The dimensionless forms are the same as in Section 4.1, Equations (4.1) and (4.2), with and without velocity as a dependent variable respectively, hence they are not repeated here for the sake of brevity.

\subsubsection{Thermoviscoelastic Solids without Memory: $\mathrm{R}^{1}$}

The mathematical model in Section 4.2 (Equations (4.5)) can be nondimensionalized using (4.7). The resulting dimensionless forms of the equations are

$$
\begin{aligned}
& \rho_{0}=\left(f \frac{\partial u}{\partial x}+1\right) \rho(x, t) \\
& \rho_{0} \frac{\partial^{2} u}{\partial t^{2}}-\left(\frac{\tau_{0}}{\left(\tilde{\rho}_{0} v_{0}^{2}\right)}\right) \frac{\partial}{\partial x}\left(\left(f \frac{\partial u}{\partial x}+1\right) \sigma_{x x}^{[0]}\right)=0 \\
& \sigma_{x x}^{[0]}=\left(\frac{E_{0}}{\tau_{0}}\right) E\left(\varepsilon_{[0]}\right)_{x x}+c\left(\dot{\varepsilon}_{[0]}\right) ; c=\frac{\hat{c}}{\left(\tau_{0} t_{0}\right)} \\
& \left(\varepsilon_{[0]}\right)_{x x}=\frac{\partial u}{\partial x}+f \frac{1}{2}\left(\frac{\partial u}{\partial x}\right)^{2} \\
& \rho_{0} c_{p 0} \frac{\partial \theta}{\partial t}+\left(\frac{k_{0} t_{0}}{\tilde{\rho}_{0} L_{0}^{2} \tilde{c}_{p 0}}\right) \frac{\partial q}{\partial x}-\frac{2}{3}\left(\frac{\tau_{0}}{\tilde{\rho}_{0} \tilde{c}_{p 0} \theta_{0}}\right) \sigma_{x x}^{[0]}\left(\dot{\varepsilon}_{[0]}\right)_{x x}=0 \\
& q+k \frac{\partial \theta}{\partial x}=0
\end{aligned}
$$

The dimensionless modulus of elasticity is given by $E$ and $c$ is the dimensionless dissipation coefficient. For small deformation and finite deformation we use $f=0$ and $f=1$ respectively. In (4.8), we can also introduce velocity, $v$, as an additional dependent variable with the additional equation $v=\frac{\partial u}{\partial t}$ and $\frac{\partial^{2} u}{\partial t^{2}}$ replaced by $\frac{\partial v}{\partial t}$.

\subsubsection{Thermoviscoelastic Solids with Memory: $\mathbf{R}^{1}$}

The mathematical model in Section 4.3 (Equation (4.6)) can be nondimensionalized using (4.7). The resulting dimensionless form of the equations are

$$
\begin{aligned}
& \rho_{0}=\left(f \frac{\partial u}{\partial x}+1\right) \rho(x, t) \\
& \rho_{0} \frac{\partial^{2} u}{\partial t^{2}}-\left(\frac{\tau_{0}}{\left(\tilde{\rho}_{0} v_{0}^{2}\right)}\right) \frac{\partial}{\partial x}\left(\left(f \frac{\partial u}{\partial x}+1\right) \sigma_{x x}^{[0]}\right)=0 \\
& \sigma_{x x}^{[0]}+D e \frac{\partial \sigma_{x x}^{[0]}}{\partial t}=\left(\frac{E_{0}}{\tau_{0}}\right) E\left(\varepsilon_{[0]}\right)_{x x}+c\left(\dot{\varepsilon}_{[0]}\right)_{x x} \\
& \left(\varepsilon_{[0]}\right)_{x x}=\frac{\partial u}{\partial x}+f \frac{1}{2}\left(\frac{\partial u}{\partial x}\right)^{2} \\
& \rho_{o} c_{p 0} \frac{\partial \theta}{\partial t}+\left(\frac{k_{0} t_{0}}{\tilde{\rho}_{0} L_{0}^{2} \tilde{c}_{p 0}}\right) \frac{\partial q}{\partial x}-\frac{2}{3}\left(\frac{\tau_{0}}{\tilde{\rho}_{0} \tilde{c}_{p 0} \theta_{0}}\right) \sigma_{x x}^{[0]}\left(\dot{\varepsilon}_{[0]}\right)_{x x}=0 \\
& q+k \frac{\partial \theta}{\partial x}=0
\end{aligned}
$$


where Deborah number $D e=\frac{\lambda}{t_{o}}$.

\section{Computational Framework for Numerical Simulation of Evolution}

The mathematical models described in Sections 3 and 4 are a system of nonlinear partial differential equations (for finite strain measures) describing evolutions i.e. these are initial value problems (IVPs). Even in $\mathrm{R}^{1}$, the equations are complex enough not to permit theoretical or analytical solutions. In the present work, we consider a space-time coupled finite element formulation based on space-time residual functional for an increment of time with time marching for computing evolutions. The space-time local approximations are considered in higher order scalar product spaces that permit higher order global differentiability in space and time. Details of space-time coupled methods for IVPs, time marching, higher order global differentiability approximation spaces, space-time variationally consistent integral forms etc. can be found in references [21]-[30]. In the following we present a summary.

\subsection{Space-Time Finite Element Formulation Based on Residual Functional and the Solution Procedure}

For the sake of simplicity, we consider mathematical models in $\mathrm{R}^{1}$ describing one-dimensional wave propagation in thermoelastic and thermoviscoelastic media with and without memory. This choice is due to simplicity of physics so that the significant and subtle features of linear and non-linear wave propagation can be clearly demonstrated. Thus the mathematical models in Section $4\left(\mathrm{R}^{1}\right)$ contain $x$ and $t$ as independent coordinates. All three mathematical modes in Section 4 can be arranged in the following compact form.

$$
\boldsymbol{A} \boldsymbol{\phi}-\boldsymbol{f}=0 \quad \forall(x, t) \in \Omega_{x t}=\Omega_{x} \times \Omega_{t}=(0, L) \times(0, \tau)
$$

or

$$
A_{i} \phi_{i}-f_{i}=0 ; \quad i=1,2, \cdots, m \quad \forall(x, t) \in \Omega_{x t}
$$

Equations (5.1) or (5.2) are a system of $m$ partial differential equations. In (5.1), matrix $\boldsymbol{A}$ contains the differential operators, $\phi$ is a vector of dependent variables, and $f$ is a vector containing nonhomogeneous terms. In (5.1), $\Omega_{x t}$ is the open space-time domain such that $\bar{\Omega}_{x t}=\Omega_{x t} \cup \Gamma, \bar{\Omega}_{x t}$ being closure of $\Omega_{x t}$ and $\Gamma$ being the closed boundary of $\Omega_{x t}$. Additionally, the following holds (Figure 1), $\bar{\Omega}_{x}=\Omega_{x} \cup \Gamma_{x}$ and $\bar{\Omega}_{t}=\Omega_{t} \cup \Gamma_{t}$ such that $\Gamma=\Gamma_{x} \cup \Gamma_{t}$. For simplicity, we consider $\Gamma=\bigcup_{i=1}^{4} \Gamma_{i}$ as shown in Figure 1(a). Figure 1(b) shows a subdivision of the space-time domain $\bar{\Omega}_{x t}$ into space-time strips such that

$$
\bar{\Omega}_{x t}=\bigcup_{n}^{n} \bar{\Omega}_{x t} \quad \forall(x, t) \in{ }^{n} \bar{\Omega}_{x t}=\bar{\Omega}_{x} \times{ }^{n} \bar{\Omega}_{t}=[0, L] \times\left[t_{n-1}, t_{n}\right]
$$

The $n^{\text {th }}$ space-time strip, with domain ${ }^{n} \bar{\Omega}_{x t}$, is from time $t_{n-1}$ to $t_{n}$ over the spatial domain $[0, L]$. The time interval $\Delta t$ for the strips need not be uniform (but assume so here for simplicity). Consider the $\mathrm{n}^{\text {th }}$ spacetime strip ${ }^{n} \bar{\Omega}_{x t}$ and its discretization ${ }^{n} \bar{\Omega}_{x t}^{\mathrm{T}}$ into space-time elements

$$
{ }^{n} \bar{\Omega}_{x t}^{\mathrm{T}}=\bigcup_{e} \bar{\Omega}_{x t}^{e}
$$

in which $\bar{\Omega}_{x t}^{e}$ is the space-time domain of a space-time element, $e$ (Figure 1(c)), a nine node space-time p-version element. Consider the $n^{\text {th }}$ space-time strip with its space-time domain ${ }^{n} \bar{\Omega}_{x t}$ and its discretization ${ }^{n} \bar{\Omega}_{x t}^{\mathrm{T}}$. Let ${ }_{i}^{n} \phi_{h} ; i=1,2, \cdots, m$ be the approximations of $\phi_{i} ; i=1,2, \cdots, m$ over ${ }^{n} \bar{\Omega}_{x t}^{\mathrm{T}}$ and let ${ }_{i}^{n} \phi_{h}^{e}$ be the local approximation of $\phi_{i}$ over a space-time element $\bar{\Omega}_{x t}^{e}$ such that

$$
{ }_{i}^{n} \phi_{h}=\bigcup_{e}^{n} \phi_{h}^{e} ; i=1,2, \cdots, m
$$

If we substitute ${ }_{i}^{n} \phi_{h}$ in (5.2), then we obtain the residual functions (equations), $E_{i}=i=1,2, \cdots, m$, for the $n^{\text {th }}$ space-time strip.

$$
E_{i}=A_{i}\left(\begin{array}{l}
n \\
i
\end{array} \phi_{h}\right)-f_{i} ; i=1,2, \cdots, m
$$




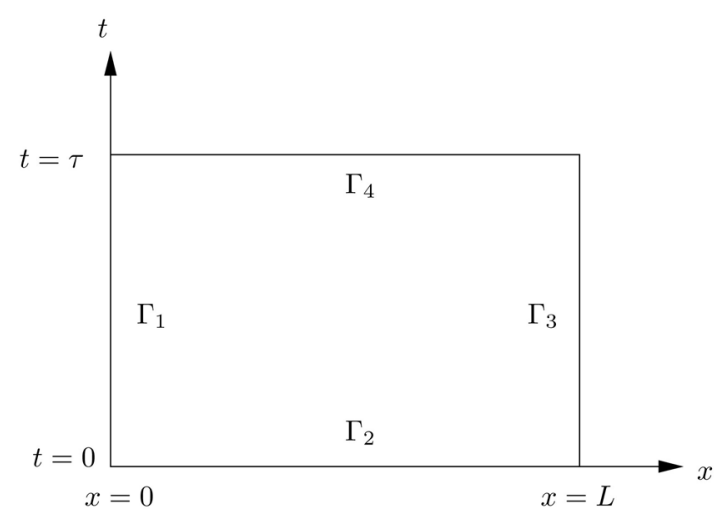

(a)

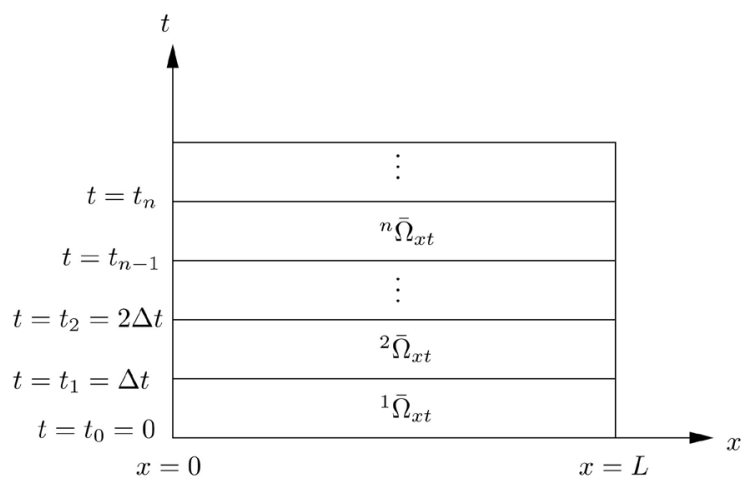

(b)

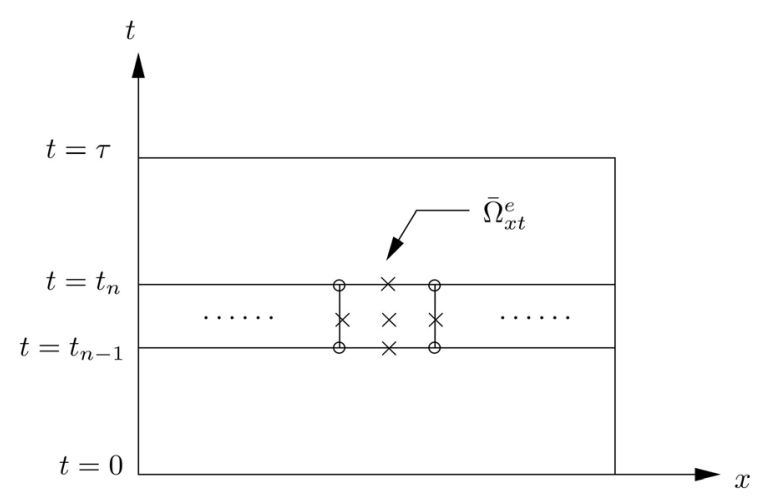

(c)

Figure 1. Space-time domain, space-time strips, and discretization for nth space-time strip. (a) Space-time domain; (b) Space-time strips ${ }^{k} \bar{\Omega}_{x t}^{T} ; k=1,2, \cdots, n, \cdots ;$ (c) Discretization for $n^{\text {th }}$ space-time strip.

On the other hand, if we substitute ${ }_{i}^{n} \phi_{h}^{e}$ in (5.2), we obtain residual equations, $E_{i}^{e}$, for a space-time element e.

$$
E_{i}^{e}=A_{i}\left(\begin{array}{c}
n \\
i
\end{array} \phi_{h}^{e}\right)-f_{i} ; i=1,2, \cdots, m
$$

We consider the space-time finite element method based on residual functional (space-time least squares method). See references [21]-[30] for more details. Let ${ }^{n} I$ be the residual functional for the discretization of the $n^{\text {th }}$ space-time strip defined by the sum of the scalar products of $E_{i}$ with itself over ${ }^{n} \bar{\Omega}_{x t}^{\mathrm{T}}$.

$$
{ }^{n} I=\sum_{i=1}^{m}\left(E_{i}, E_{i}\right)_{{ }_{n}} \bar{\Omega}_{x t}^{\mathrm{T}}
$$

Since $\left(E_{i}, E_{i}\right)_{{ }^{n} \bar{\Omega}_{x t}^{\mathrm{T}}}$ is a functional, (5.8) can be written in terms of the sum of element residuals, i.e.

$$
{ }^{n} I=\sum_{i}\left(E_{i}, E_{i}\right)_{{ }^{n} \bar{\Omega}_{x t}^{\mathrm{T}}}=\sum_{e}\left(\sum_{i}\left(E_{i}^{e}, E_{i}^{e}\right)_{\bar{\Omega}_{x t}^{e}}\right)=\sum_{e} I^{e}
$$

Based on the calculus of variations [21], an extremum of the functional ${ }^{n} I$ is also a solution of the associated Euler's equations (partial differential equations in the mathematical models). An extremum of ${ }^{n} I$ requires that we set its first variation, $\delta\left({ }^{n} I\right)$, to zero, a necessary condition, provided ${ }^{n} I$ is differentiable in its arguments.

$$
\delta\left({ }^{n} I\right)=\sum_{e} \delta I^{e}=2 \sum_{e}\left(\sum_{i}\left(E_{i}^{e}, \delta E_{i}^{e}\right)_{\bar{\Omega}_{x t}^{e}}\right)=2 \sum_{e}\left\{g^{e}\right\}=2\{g\}=0
$$

Thus, $\{g\}=0$ is a necessary condition for an extremum of functional ${ }^{n} I$. The sufficient condition, or extremum principle, is given by 


$$
\delta^{2}\left({ }^{n} I\right)=2 \sum_{e}\left(\sum_{i}\left(\left(\delta E_{i}^{e}, \delta E_{i}^{e}\right)_{\bar{\Omega}_{x t}^{e}}+\left(E_{i}^{e}, \delta^{2} E_{i}^{e}\right)_{\bar{\Omega}_{x t}^{e}}\right)\right)
$$

In (5.11), $\delta^{2}\left({ }^{n} I\right)>0,=0,<0$, ensures a minimum, a saddle point, or a maximum, respectively, of ${ }^{n} I$ for the solution ${ }_{i}^{n} \phi_{h}$ obtained from (5.10). Equation (5.11) is clearly not an extremum principle. Following [21]-[30], we approximate (5.11) to obtain a unique extremum principle.

$$
\delta^{2}\left({ }^{n} I\right) \cong 2 \sum_{e}\left(\sum_{i}\left(\delta E_{i}^{e}, \delta E_{i}^{e}\right)_{\bar{\Omega}_{x t}^{e}}\right)>0
$$

This is a unique extremum principle (see reference [21] for details). Since some of the equations in the mathematical model are nonlinear, some $E_{i}^{e}$ are nonlinear functions of the dependent variables. That is $\{g\}$ in (5.10) is a nonlinear function. Consider the local approximations ${ }_{i}^{n} \phi_{n}^{e} \in V_{n} \subset H^{k, p}\left(\bar{\Omega}_{x t}^{e}\right)$ in which $k=\left(k_{1}, k_{2}\right), k_{1}$ and $k_{2}$ being the orders of the scalar product space $H^{k, p}\left(\bar{\Omega}_{x t}^{e}\right)$ in space and time. Consider the local approximations of $\phi_{i}$ over $\bar{\Omega}_{x t}^{e}$

$$
{ }_{i}^{n} \phi_{h}^{e}=\left[N^{i}\right]\left\{{ }_{i} \delta^{e}\right\} ; i=1,2, \cdots, m
$$

in which $\left[N^{i}\right]$ are space-time local approximation functions and $\left\{{ }_{i} \delta^{e}\right\}$ are nodal degrees of freedom for a dependent variable $\phi_{i}$. Let $\left\{\delta^{e}\right\}^{\mathrm{T}}=\left[\left\{{ }_{1} \delta^{e}\right\}^{\mathrm{T}},\left\{{ }_{2} \delta^{e}\right\}^{\mathrm{T}}, \cdots,\left\{{ }_{m} \delta^{e}\right\}^{\mathrm{T}}\right]$ be the total degrees of freedom for all of the dependent variables $\phi_{i}$ for an element, $e$. Therefore, the total degrees of freedom $\{\delta\}$ for the discretization ${ }^{n} \bar{\Omega}_{x t}^{\mathrm{T}}$ can be written as

$$
\{\delta\}=\bigcup_{e}\left\{\delta^{e}\right\}
$$

With (5.13) and (5.14), $\{g\}$ in (5.10) is a nonlinear function of $\{\delta\}$, hence the necessary condition $\{g\}=0$ must be satisfied iteratively. We consider Newton's linear method. Let $\left\{\delta_{0}\right\}$ be an assumed solution (a starting solution), then

$$
\left\{g\left(\left\{\delta_{0}\right\}\right)\right\} \neq 0
$$

Let $\{\Delta \delta\}$ be a change in $\left\{\delta_{0}\right\}$ such that

$$
\left\{g\left(\left\{\delta_{0}\right\}+\{\Delta \delta\}\right)\right\}=0
$$

We expand $\{g\}$ in (5.16) in a Taylor series about $\left\{\delta_{0}\right\}$ and retain only up to linear terms in $\{\Delta \delta\}$.

$$
\left\{g\left(\left\{\delta_{0}\right\}+\{\Delta \delta\}\right)\right\} \cong\left\{g\left(\left\{\delta_{0}\right\}\right)\right\}+\left.\frac{\partial\{g\}}{\partial\{\delta\}}\right|_{\left\{\delta_{0}\right\}}\{\Delta \delta\}=0
$$

Then

$$
\{\Delta \delta\}=-\left[\frac{\partial\{g\}}{\partial\{\delta\}}\right]_{\left\{\delta_{0}\right\}}^{-1}\left\{g\left(\left\{\delta_{0}\right\}\right)\right\}
$$

An improved solution, $\{\delta\}$, is obtained using

$$
\{\delta\}=\left\{\delta_{0}\right\}+\alpha\{\Delta \delta\} ; 0 \leq \alpha \leq 2 \text { such that }{ }^{n} I(\{\delta\}) \leq{ }^{n} I\left(\left\{\delta_{0}\right\}\right)
$$

Use of $\alpha$ in (5.19) is called line search [21]-[30]. Using $\{\delta\}$ in (5.19), we check if the absolute value of each component of $\{g(\{\delta\})\}$ is less than or equal to $\Delta$, (generally $10^{-6}$ or lower) a preset tolerance for computed zero. If this condition is satisfied by $\{\delta\}$ in (5.19), then we have a converged solution $\{\delta\}$ from Newton's linear method, otherwise we set $\left\{\delta_{0}\right\}$ to be $\{\delta\}$ and repeat (another iteration) the calculations described above. It is worth noting that 


$$
\frac{\partial\{g\}}{\partial\{\delta\}} \mid=\frac{1}{2} \delta^{2} I
$$

which when approximated using (5.12) gives a positive definite coefficient matrix due to the fact that $\delta^{2} I>0$. Thus, we can rewrite (5.18) as

$$
\begin{gathered}
\{\Delta \delta\}=-\frac{1}{2}\left[\delta^{2} I\right]_{\left\{\delta_{0}\right\}}^{-1}\left\{g\left(\left\{\delta_{0}\right\}\right)\right\} \\
\delta^{2} I=\sum_{e}\left(\sum_{i}\left(\delta E_{i}^{e}, \delta E_{i}^{e}\right)_{\bar{\Omega}_{x t}^{e}}\right)=\sum_{e}\left[K^{e}\right]
\end{gathered}
$$

in which $\left[K^{e}\right]$ is the element coefficient matrix and $\left[\delta^{2} I\right]$ in (5.19) are the assembled element equations for the discretization ${ }^{n} \bar{\Omega}_{x t}^{\mathrm{T}}$. Likewise, the following holds

$$
\{g\}=\sum_{e}\left\{g^{e}\right\} ; \quad\left\{g^{e}\right\}=\sum_{i}\left(E_{i}^{e}, \delta E_{i}^{e}\right)
$$

\subsection{Time Marching Procedure: Computations of Evolution}

We initiate computations with the first space-time strip shown in Figure 2 with boundary conditions on two boundaries (for example) and initial conditions at time $t=0$, the boundary at $t=\Delta t$ being the open boundary where nothing is known about the solution. With proper choice of discretization, p-level, and minimally conforming space choice [21]-[30], the integrated sum of squares of the residuals ${ }^{1} I$ for the first space-time strip are achieved to be less than or equal to $O\left(10^{-6}\right)$. With the minimally conforming choice of $k$, the orders $k_{1}$ and $k_{2}$ of the approximation space in space and time, the space-time integrals are Riemann over ${ }^{n} \bar{\Omega}_{x t}^{\mathrm{T}}$, hence ${ }^{1} I$ of the order of $O\left(10^{-6}\right)$ or lower indicates that the GDEs are satisfied accurately in the pointwise sense over ${ }^{1} \bar{\Omega}_{x t}^{\mathrm{T}}$ [21]-[30]. Upon obtaining an accurate solution for ${ }^{1} \bar{\Omega}_{x t}^{\mathrm{T}}$ the computations are initiated for ${ }^{2} \bar{\Omega}_{x t}^{\mathrm{T}}$ keeping the same p-levels, same values of $k$, and the same discretization as used for ${ }^{1} \bar{\Omega}_{x t}^{\mathrm{T}}$. For the second space-time strip, ${ }^{2} \bar{\Omega}_{x t}^{\mathrm{T}}$, ICs at $t=\Delta t$ are from the computed solution at $t=\Delta t$ for ${ }^{1} \bar{\Omega}_{x t}^{\mathrm{T}}$. This process is continued till the desired time $t=\tau$ is reached. The benefits of space-time coupled finite element process based on residual functional and the computations of evolutions using space-time strip with time marching are well documented in references [21]-[30].

\section{Model Problems}

We consider one-dimensional axial wave propagation in thermoelastic solid continua and thermoviscoelastic solid continua with and without memory. In all three mathematical models (Section 4.4) Green's strain tensor is used as a measure of finite strain and the second Piola-Kirchoff stress tensor as energy conjugate stress measure. Figure 3(a) shows a schematic of the dimensionless rod of length one unit. The fixed end at $x=0$ is also the

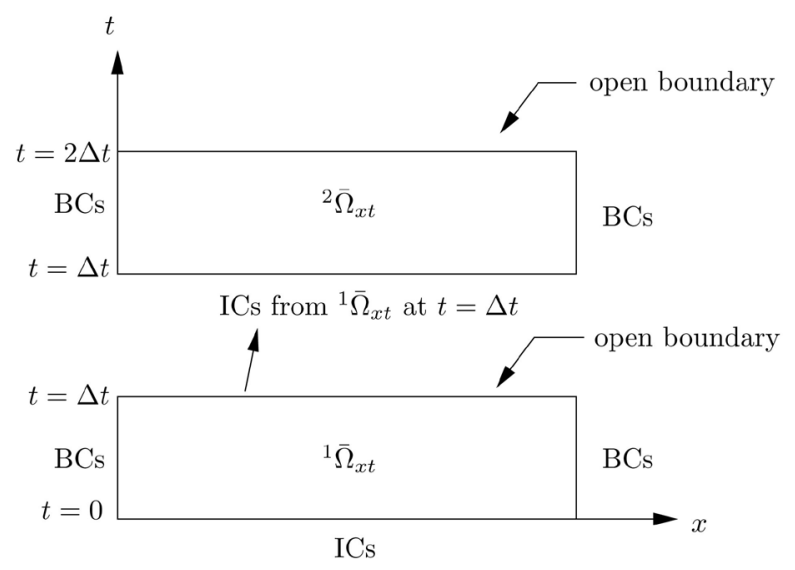

Figure 2. First two space-time strips with BCs and ICs. 


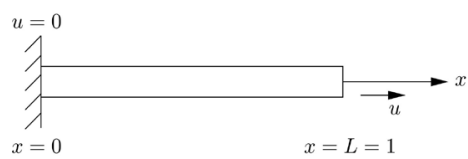

(a)

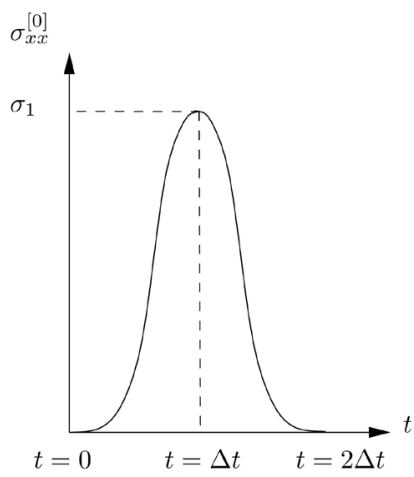

(b)

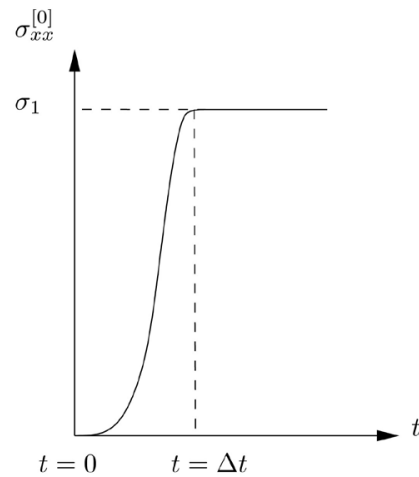

(c)

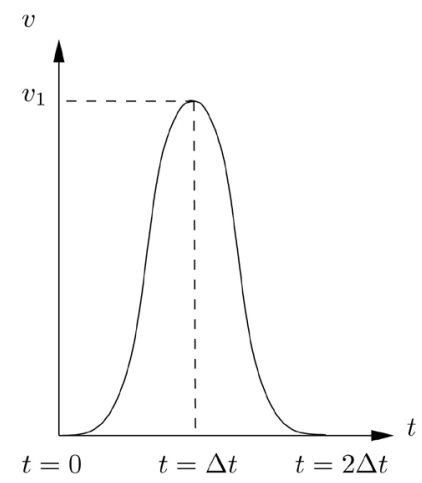

(d)

Figure 3. Problem schematic, stress pulse, stress ramp, and velocity pulse loading. (a) Schematic; (b) Stress pulse loading: L1; (c) Stress ramp loading: L2; (d) Velocity pulse loading: L3.

origin of the $x$-frame. The dimensionless axial rod is of unit length. The right end of the rod (at $x=1.0$ ) is subjected to three different types of loading.

\subsection{Loadings}

We consider three different types of loads applied to the end of the rod at $x=1.0$.

\section{Loading L1:}

This loading consists of a stress pulse $\sigma_{x x}^{[0]}(t)$ of maximum amplitude $\pm \sigma_{1}$, positive for tensile loading and negative for compressive loading applied over a time interval of $2 \Delta t$. In Figure $3(\mathbf{b}), \sigma_{x x}^{[0]}(t)$ is continuous with continuous first time derivative for $0 \leq t \leq 2 \Delta t$ and is defined using the following.

$$
\begin{aligned}
& \text { at } t=0 ; \sigma_{x x}^{[0]}(t)=0, \frac{\partial \sigma_{x x}^{[0]}}{\partial t}=0 \\
& \text { at } t=\Delta t ; \sigma_{x x}^{[0]}(t)= \pm \sigma_{1}, \frac{\partial \sigma_{x x}^{[0]}}{\partial t}=0 \\
& \text { at } t=2 \Delta t ; \sigma_{x x}^{[0]}(t)=0, \frac{\partial \sigma_{x x}^{[0]}}{\partial t}=0 \\
& \text { for } t \geq 2 \Delta t ; \sigma_{x x}^{[0]}(t)=0
\end{aligned}
$$

The stress pulse $\sigma_{x x}^{[0]}(t)$ described by (6.1) has support of $2 \Delta t$ with maximum amplitude of $\pm \sigma_{1}$ at $t=\Delta t$ such that for $0 \leq t \leq 2 \Delta t \quad \sigma_{x x}^{[0]}(t)$ is a cubic function of time $t$ and $\sigma_{x x}^{[0]}(t)=0$ for $t \geq 2 \Delta t$.

Loading L2:

This loading consists of stress $\sigma_{x x}^{[0]}(t)$ defined as a ramp function over a time interval of $\Delta t$ with maximum value of $\pm \sigma_{1}$. Positive and negative signs correspond to tension and compression respectively. The ramp $\sigma_{x x}^{[0]}(t)$ is continuous with continuous first derivative for $0 \leq t \leq \Delta t$ and remains $\pm \sigma_{1}$ (constant magnitude) for $t \geq \Delta t$.

$$
\begin{aligned}
& \text { at } t=0 ; \sigma_{x x}^{[0]}(t)=0, \frac{\partial \sigma_{x x}^{[0]}}{\partial t}=0 \\
& \text { at } t=\Delta t ; \sigma_{x x}^{[0]}(t)= \pm \sigma_{1}, \frac{\partial \sigma_{x x}^{[0]}}{\partial t}=0 \text { for } t \geq \Delta t ; \sigma_{x x}^{[0]}(t)= \pm \sigma_{1}
\end{aligned}
$$


The ramp $\sigma_{x x}^{[0]}(t)$ described by (6.2) is a stress loading with maximum value of $\pm \sigma_{1}$ such that for $0 \leq t \leq \Delta t, \sigma_{x x}^{[0]}(t)$ is a cubic function of $t$ with zero time derivatives at $t=0$ and at $t=\Delta t$ and a constant value of $\pm \sigma_{1}$ for $t \geq \Delta t$. Figure 3(c) shows a schematic of this loading.

\section{Loading L3:}

This loading (Figure 3(d)) consists of a velocity pulse, $v(t)$, of maximum amplitude $\pm v_{1}$, positive for tensile loading and negative for compressive loading applied over a time interval of $2 \Delta t$. Similar to loading L1, we can define $v(t)$ as follows.

$$
\begin{aligned}
& \text { at } t=0 ; v(t)=0, \frac{\partial v(t)}{\partial t}=0 \\
& \text { at } t=\Delta t ; v(t)= \pm v_{1}, \frac{\partial v(t)}{\partial t}=0 \\
& \text { at } t=2 \Delta t ; v(t)=0, \frac{\partial v(t)}{\partial t}=0 \\
& \text { for } t \geq 2 \Delta t ; v(t)=0
\end{aligned}
$$

The velocity $v(t)$ described by (6.3) is a velocity pulse of support $2 \Delta t$ with maximum amplitude of $\pm v_{1}$ at $t=\Delta t$. For $0 \leq t \leq 2 \Delta t \quad v(t)$ is a cubic function of $t$ and $v(t)=0$ for $t \geq 2 \Delta t$.

\subsection{Material Coefficients, Reference Quantities and Dimensionless Parameters}

We define the material coefficients for thermoelastic sold continua and the thermoviscoelastic solid continua with and without memory, choice of reference quantities, and the resulting dimensionless material coefficients and the dimensionless variables and the parameters. The basic material is hard rubber or polymer which we would treat as thermoelastic, thermoviscoelastic without memory as well as with memory.

Thermoelastic Solid Continua (TE)

$$
\begin{gathered}
\hat{\rho}=1850 \frac{\mathrm{kg}}{\mathrm{m}^{3}} \\
\hat{E}=1.49 \times 10^{7} \frac{\mathrm{N}}{\mathrm{m}^{2}}
\end{gathered}
$$

If we choose $\tilde{\rho}_{0}=1850 \frac{\mathrm{kg}}{\mathrm{m}^{3}}$ and $E_{0}=1.49 \times 10^{7} \frac{\mathrm{N}}{\mathrm{m}^{2}}$ as reference values, then the dimensionless density $\rho_{0}=\frac{\hat{\rho}}{\tilde{\rho}_{0}}=1$ and the dimensionless modulus of elasticity $E=\frac{\hat{E}}{E_{0}}=1$.

Thermoviscoelastic Solid without Memory (TVE)

$$
\begin{gathered}
\hat{\rho}=1850 \frac{\mathrm{kg}}{\mathrm{m}^{3}} ; \quad \hat{c}_{p}=1650 \frac{\mathrm{J}}{\mathrm{kg} \cdot \mathrm{K}} \\
\hat{k}=0.235 \frac{\mathrm{W}}{\mathrm{m} \cdot \mathrm{K}} ; \quad \hat{E}=1.49 \times 10^{7} \frac{\mathrm{N}}{\mathrm{m}^{2}} \\
L_{0}=1 \mathrm{~m} ; \quad \theta_{0}=300 \mathrm{~K}
\end{gathered}
$$

reference speed of sound

$$
v_{0}=\sqrt{\frac{\hat{E}}{\hat{\rho}}}=89.7444 \frac{\mathrm{m}}{\mathrm{s}}
$$

reference time 


$$
\begin{gathered}
t_{0}=\frac{L_{0}}{v_{0}}=0.0111 \mathrm{~s} \\
\hat{\rho} v_{0}^{2}=\hat{\rho}\left(\sqrt{\frac{\hat{E}}{\hat{\rho}}}\right)^{2}=\hat{E}
\end{gathered}
$$

characteristic kinetic energy.

If we choose

$$
\tilde{\rho}_{0}=\hat{\rho}, E_{0}=\hat{E} \text {, and } \tilde{c}_{p 0}=\hat{c}_{p} \text {, then } \rho_{0}=\frac{\hat{\rho}}{\tilde{\rho}_{0}}=1 \text { and } E=\frac{\hat{E}}{E_{0}}=1 \text { and } c_{p 0}=\frac{\hat{c}_{p}}{\tilde{c}_{p 0}}=1 .
$$

\section{Thermoviscoelastic Solid with Memory (TVEM)}

The material coefficients, reference quantities, and dimensionless quantities and parameters for TVE hold here. Additionally for this solid continua we have Deborah number, $D e$, defined by $D e=\frac{\lambda}{t_{0}}$. Numerical values of $D e$ used in the evolution computations are given with the details of studies.

\subsection{Computations of Evolutions: Numerical Results}

In the following sections we report numerical studies for loading L1 and L2 for TE, TVE, and TVEM solid continua. Evolution in each case is computed using space-time strip with time marching until the desired value of time is reached. The choice of $h, p$, and $k$ defining the scalar product space $H^{p, k}\left(\bar{\Omega}_{x t}^{e}\right)$ containing space-time local approximation function is important. Since all three mathematical models (dimensionless forms given by (4.1), (4.8), and (4.9)) are a system of first order partial differential equations in space coordinate $x$ and time $t$, the choice of $k=\left(k_{1}, k_{2}\right)=(2,2)$ in space and time ensures that the local approximations are of class $C^{11}$ in space and time. Here the space-time integrals over ${ }^{n} \bar{\Omega}_{x t}^{\mathrm{T}}$, discretization of $n^{\text {th }}$ space-time strip ${ }^{n} \bar{\Omega}_{x t}$ are always Riemann. We consider a sixteen element uniform discretization of ${ }^{n} \bar{\Omega}_{x t}$ giving rise to a spatial discretization length of $1 / 16$. With $E=1, \rho_{0}=1$, the dimensionless wave speed is one, thus with $\Delta t=0.1$, the wave would be over a spatial domain of 0.1 which is spanned by approximately one and a half space-time element. Hence, at the onset, the sixteen element uniform spatial discretization $\left(h_{e}=1 / 16\right)$ appears to be reasonable. With $k=\left(k_{1}, k_{2}\right)=(2,2)$ and $h_{e}=1 / 16$, we need to conduct a $p$-convergence study to establish at what $p$-levels this choice of $h_{e}$ is adequate to yield values of the residual functional for the space-time strip low enough for the computed solution to be considered accurate or time accurate. For this purpose, we consider the first space-time strip with loading L2 and $\sigma_{1}= \pm 0.01$ and $\sigma_{1}= \pm 0.1$ at $x=1.0$ and $\Delta t=0.1$. The $p$-levels in space and time, $\left(p_{1}, p_{2}\right)$, are increased uniformly $\left(p=p_{1}=p_{2}\right)$ from 3 to 11 in increments of 2 . For each $p$-level, a solution is computed using a tolerance $\Delta=10^{-6}$ for $\left|g_{i}\right| \leq \Delta, i=1,2, \cdots$ in Newton's linear method with line search. The behavior of the residual function $I$ for ${ }^{1} \bar{\Omega}_{x t}^{\mathrm{T}}$ is examined as a function of the degrees of freedom for TE, TVE solids and for TVEM. Plots of residual function $I$ versus degrees of freedom for TE, TVE, and TVEM for both linear and nonlinear cases corresponding to infintesimal (linear) and finite strain formulations (nonlinear) are given in Figure 4. In the mathematical models, $f=0$ is used for the linear case in which there is no non-linearity in any of the equations in the mathematical model. When $f=1$ (nonlinear case), the strain measure is Green's strain and $\rho(x, t) \neq \rho_{0}(x)$, instead $\rho_{0}=\left(\frac{\partial u}{\partial x}+1\right) \rho(x, t)$ holds due to the continuity equation. From the graphs in Figure 4, we note that: 1) in all three cases (TE, TVE, and TVEM) the residual $I$ is of the order of $O\left(10^{-12}\right)$ or lower for $p=p_{1}=p_{2}=9$ or greater confirming that $h_{e}=1 / 16$, $k_{1}=k_{2}=2$, and $p_{1}=p_{2}=9$ are sufficient for accurate solution for the first space-time strip. For the second space-time strip, ICs at $t=\Delta t$ are obtained from the solution for the first space-time strip at $t=\Delta t$. For these choices of $h, p$, and $k$, the evolution is expected to stay accurate as long as $I$ of the order of $O\left(10^{-12}\right)$ or lower is achieved. This in fact is the assurance of good accuracy of the computed evolution. 2) Even though the slopes of the $I$ vs dof graphs vary slightly in Figure 4(b) and Figure 4(c), for all practical purposes the change is not significant, hence we can conclude that the rate of convergence (in the asymptotic range) is almost the same in each plot of $I$ shown in Figures 4(a)-(c). 


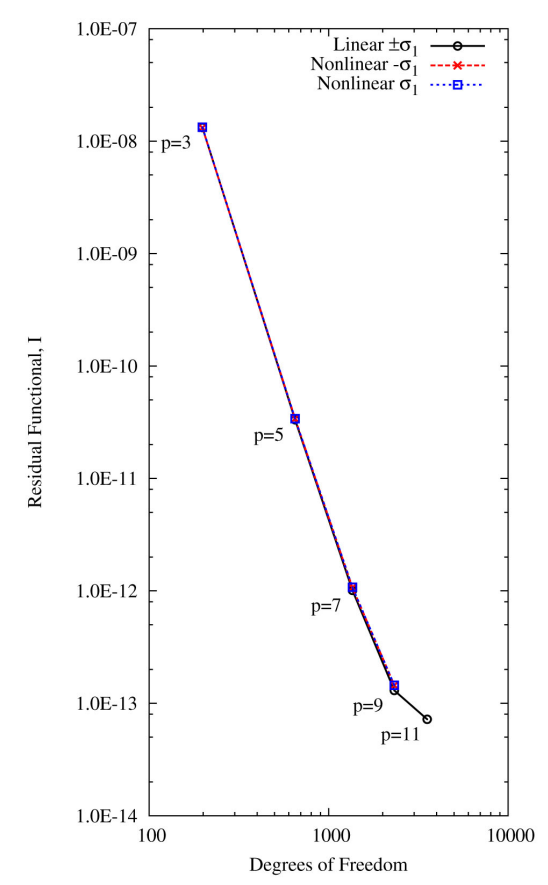

(a)

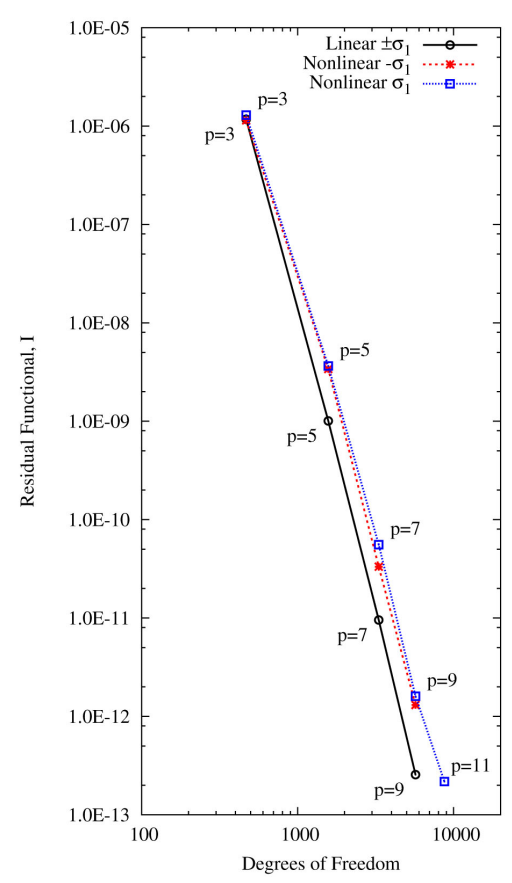

(b)

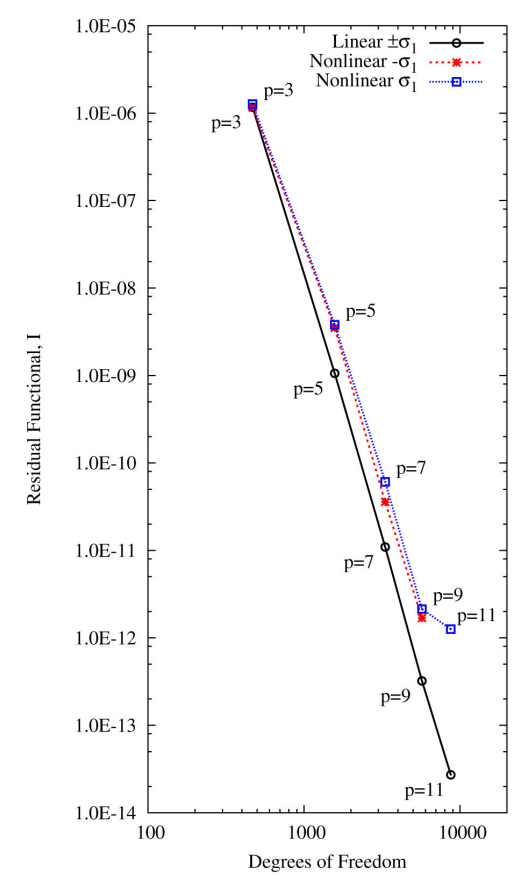

(c)

Figure 4. Convergence of residual functional I: I versus dof. (a) TE; (b) TVE; (c) TVEM.

\subsubsection{Linear and Nonlinear Waves in TE Solid Continua}

In this section, we present computed evolutions for TE solid continua for linear and nonlinear cases. In linear wave propagation with infintesimal deformation, there is no change in density and the stress $\sigma_{x x}^{[0]}=\sigma_{x x}^{(0)}=\sigma_{x x}$ and is a linear function of $\frac{\partial u}{\partial x}$, and $\frac{\partial u}{\partial x} \ll 1$, hence $\rho_{0}=\rho(x, t)$ holds during evolution. When considering compressive $\sigma_{x x}^{[0]}$ at $x=1$, for nonlinear case, caution should be exercised regarding the magnitude of $-\sigma_{1}$ as for this case for some value of $-\sigma_{1}$ the stiffness due to $\sigma_{x x}^{[0]}$ will become equal to the nonlinear stiffness of the rod causing instability, hence failure of computations [31]-[33]. This will occur at the fixed end during reflection when the magnitude of the stress momentarily jumps (double in linear case). In the present studies for TE solid continua, we choose $\sigma_{1}= \pm 0.01$ for loading L1 as well as loading L2, well below the stress value that causes instability. In all computations, constant $\Delta t=0.1$ is maintained.

\section{Loading L1}

\section{(a) Compressive}

We consider a compressive stress pulse with $\sigma_{1}=-0.01$. When $f=0$ i.e. linear case, the stress pulse propagates without amplitude decay and base elongation as expected due to reversibility of the deformation process. Figures 5(a)-(f) show stress wave propagation over $0 \leq x \leq 1$ for $t=5 \Delta t, 9 \Delta t, 11 \Delta t, 17 \Delta t$, and $23 \Delta t$. At $t=11 \Delta t$, the stress pulse is reflecting from the impermeable boundary at $x=0$. Exploded view of the pulse reflection at $x=0$ for $t=11 \Delta t$ is shown in Figure 5(d). Upon reflection, the reflected pulse propagates back toward the right end of the rod $(x=1.0)$ and reflects from the free boundary at $x=1.0$. This reflected stress pulse now propagates toward the left end of the rod (Figure 14(f) at $t=23 \Delta t$ ). We observe that the amplitude of the stress pulse and its base are maintained during propagation and repeated reflections as expected.

When $f=1$, nonlinear wave propagation, the material experiences compression, hence increase in density in the deformed portions of the rod which results in reduced wave speed. From Figures 5(a)-(f), we note that the nonlinear wave also maintains its support and its amplitude during propagation and reflections, but lags the linear case due to reduced wave speed compared to linear case.

Figures 6(a)-(f) show plots of velocity $v$ over $0 \leq x \leq 1$ for the same values of time as in Figures 5(a)-(f) for the compressive pulse. Here also we observe the same features for $v$ versus $x$ for various values of time as in Figures 5(a)-(f), namely, the velocity pulse remains unchanged during evolution and the nonlinear velocity 


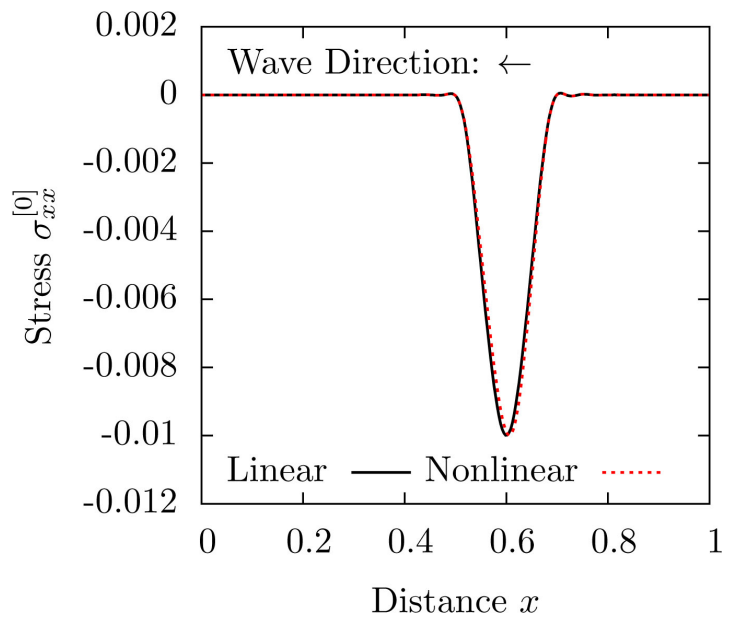

(a)

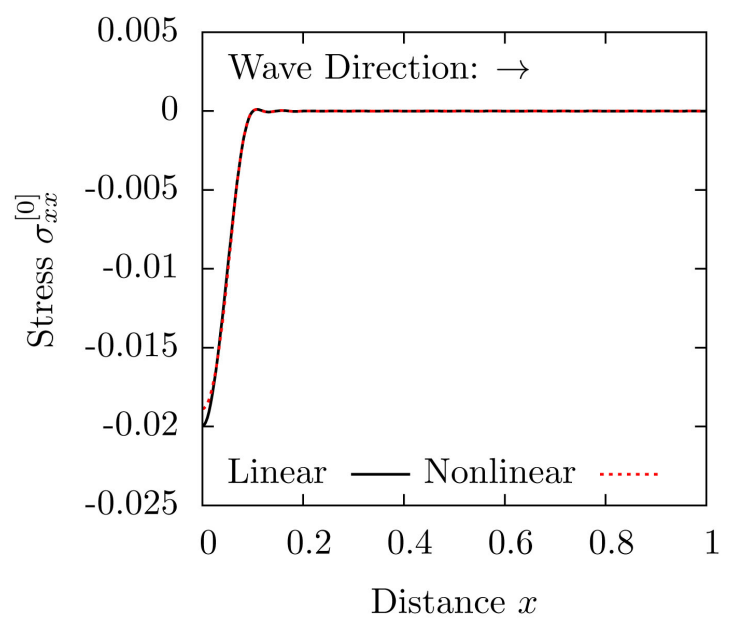

(c)

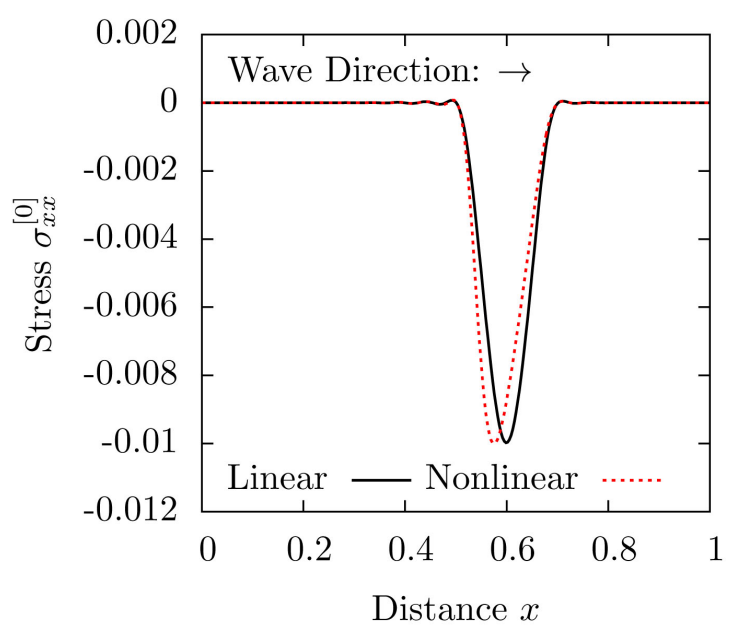

(e)

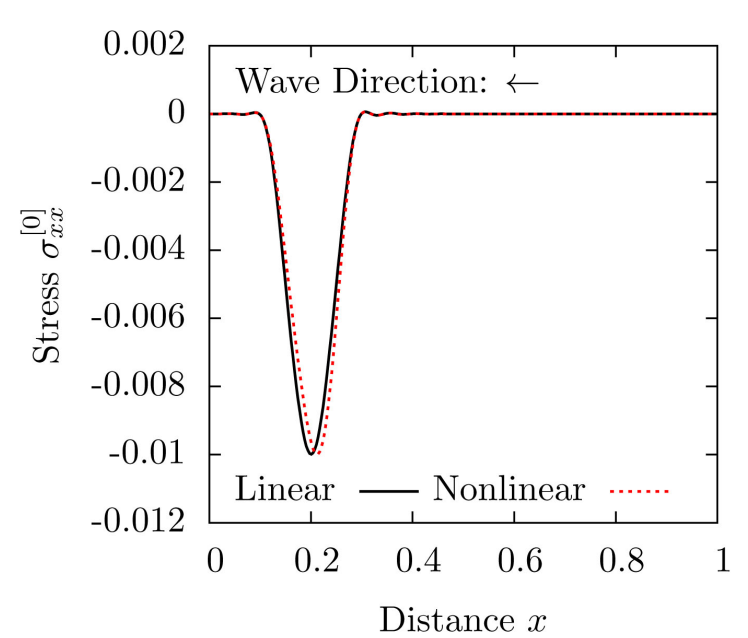

(b)

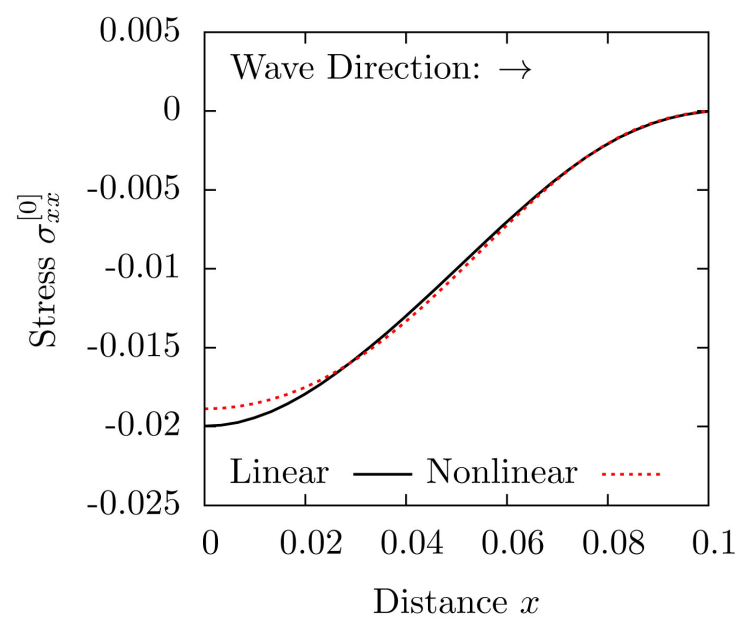

(d)

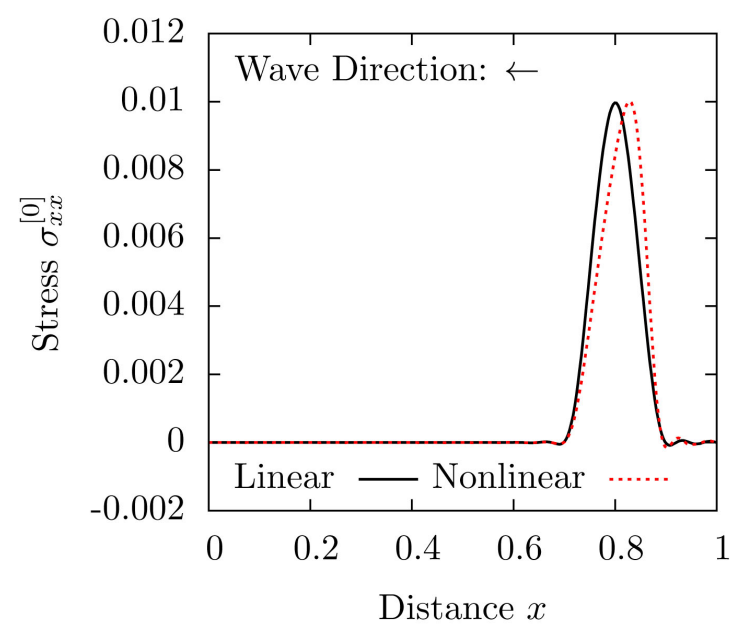

(f)

Figure 5. Evolution of $\sigma_{x x}^{[0]}$ along the length of the rod: TE, L1, $\Delta t=0.1, \sigma_{1}=-0.01$. (a) $t=5 \Delta t$; (b) $t=9 \Delta t$; (c) Reflection, $t=11 \Delta t$; (d) Details of reflection, $t=11 \Delta t$; (e) $t=17 \Delta t$; (f) $t=23 \Delta t$. 


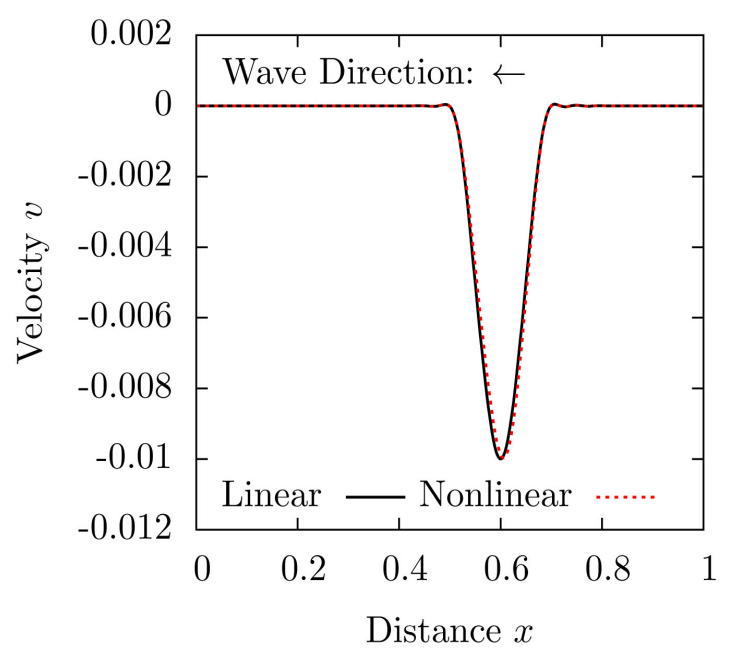

(a)

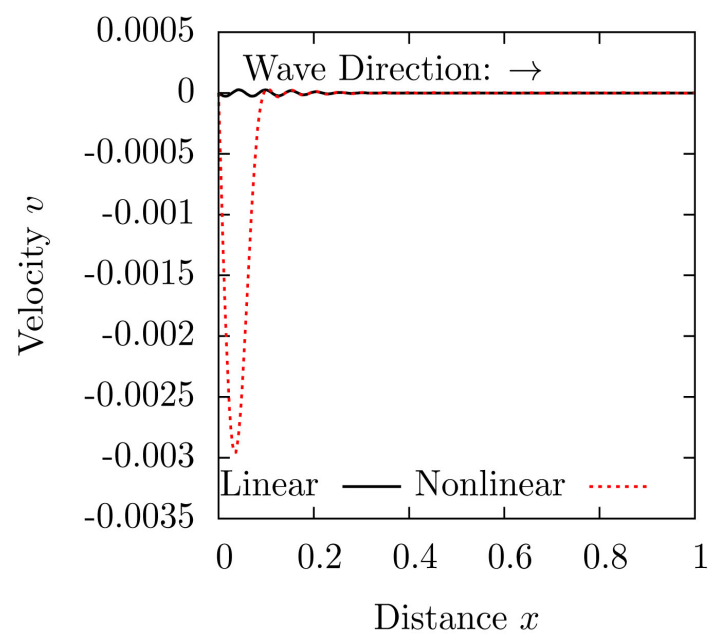

(c)

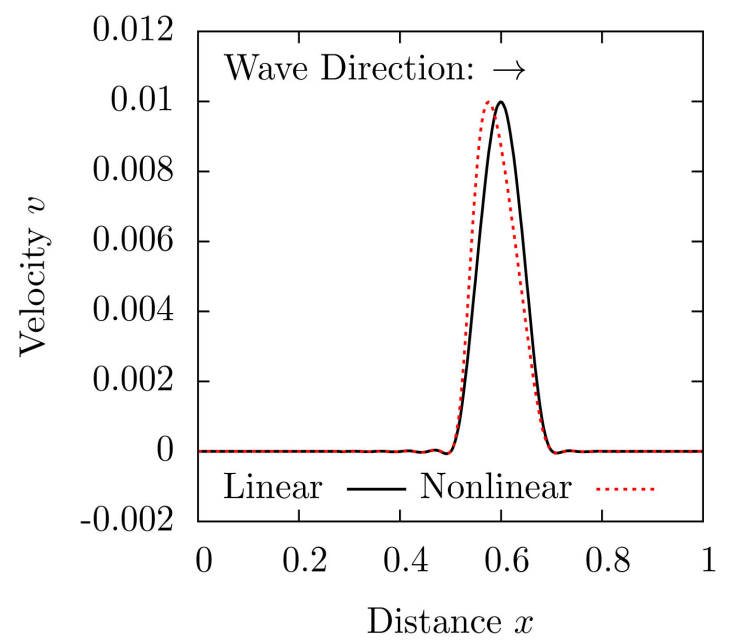

(e)

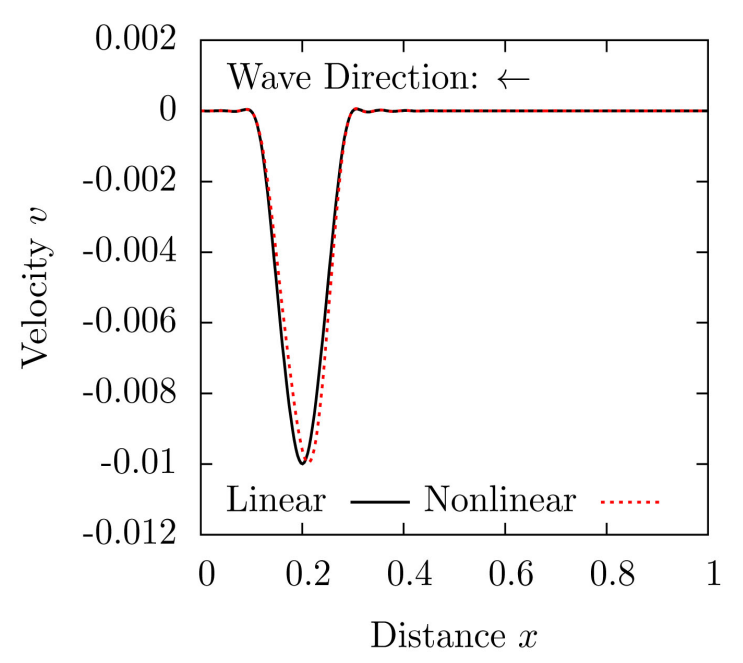

(b)

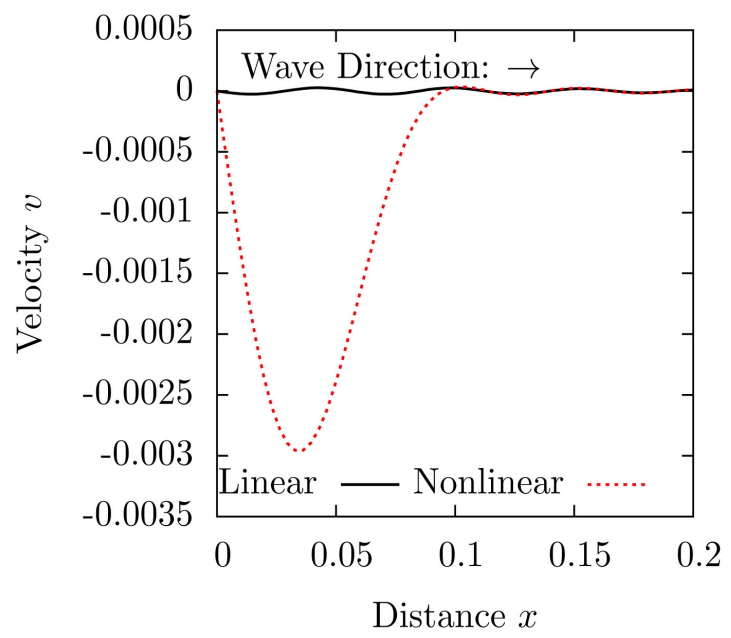

(d)

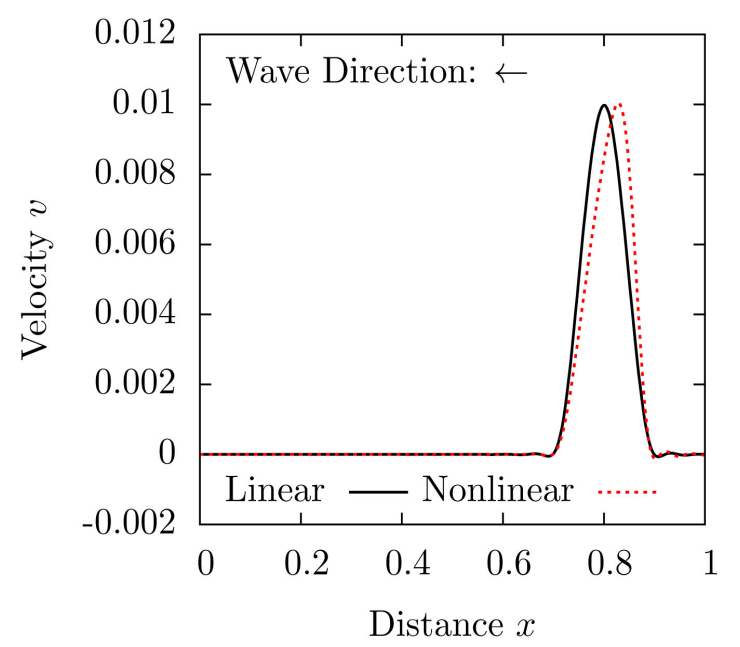

(f)

Figure 6. Evolution of $\sigma_{x x}^{[0]}$ along the length of the rod: TE, L1, $\Delta t=0.1, \sigma_{1}=-0.01$. (a) $t=5 \Delta t$; (b) $t=9 \Delta t$; (c) Reflection, $t=11 \Delta t$; (d) Details of reflection, $t=11 \Delta t$; (e) $t=17 \Delta t$; (f) $t=23 \Delta t$. 
wave lags the linear case. Most dramatic is the reflection of the velocity wave shown in Figure 6(c) and its exploded view in Figure 6(d). Dramatically different behaviors for linear and nonlinear waves are clearly observed, yet upon further evolution, the wave shape is recovered (Figure 6(e) for $t=17 \Delta t$ ).

(b) Tensile

In this study, we choose a tensile stress pulse with $\sigma_{1}=0.01$ applied at $x=1.0$. Computed evolutions for linear and nonlinear cases are shown in Figures 7(a)-(f) for the same values of time, $t$, as used in Figures 5(a)-(f). For both linear and nonlinear cases ( $f=0$ and $f=1$ ), the wave shape is preserved during propagation and the reflections from the boundaries at $x=0$ and $x=1$ take place as expected. When $f=1$ (nonlinear tensile wave), then the material density reduces due to elongation, hence increasing the local wave speed. Thus, in Figures 7(a)-(f) we observe that the nonlinear wave leads the linear wave throughout the evolution. The velocity pulse evolution for this case is similar to compressive case (except the signs). The significantly different behaviors of linear and nonlinear velocity pulses at reflection from the boundardy at $x=0$ is observed here also. This is quite similar to the reflection shown in Figure 6(c) and Figure 6(d), hence not repeated.

\section{Loading L2}

\section{(a) Compressive}

In this study, we consider loading L2 with $\sigma_{1}=-0.01$, a ramp loading over $0 \leq t \leq \Delta t$ that is of class $C^{1}$ in time. Here also we consider $f=0$ (linear wave) as well as $f=1$ (nonlinear case). When $f=0$, the magnitude of $\sigma_{1}$ remains constant and its support $\Delta t$, also remains constant i.e. no amplitude decay and base elongation. Figures 8(a)-(f) show propagation of stress wave over $0 \leq x \leq 1$ for $t=5 \Delta t, 9 \Delta t, 11 \Delta t, 17 \Delta t$, and $23 \Delta t$. At $t=11 \Delta t$, the stress wave is reflecting from the impermeable boundary at $x=0$. Exploded view of reflection at $t=11 \Delta t$ is shown in Figure 8(d). Upon reflection, the reflected stress wave propagates back toward the right end boundary at $x=1.0$ and reflects from the free boundary at $x=1.0$. The reflected stress wave now propagates back toward the left end of the rod at $x=0.0$ (Figure 8(f) at $t=23 \Delta t$ ). We observe that the amplitude of the stress wave and its support (base) are maintained during propagation and after reflection as expected in the thermoelastic solid continua. When $f=1$, the waves are nonlinear compressive as the mathematical model consists of nonlinear partial differential equations. Due to compression, the density increases in the deformed portion of the medium, hence the wave speed is reduced. From Figures 8(a)-(f), we note that the nonlinear stress wave also maintains the amplitude and the support during evolution but lags the linear case due to reduced wave speed compared to linear case. The velocity evolution shows similar features as the stress waves, but drastically different behaviors for linear and nonlinear case when reflecting from the impermeable boundary at $x=0$ (similar to Figure 6(c) and Figure 6(d)) but are not reported here for the sake of brevity.

\section{(b) Tensile}

In this study, we consider tensile stress loading with $\sigma_{1}=0.01$ applied at $x=1.0$ over $\Delta t$. Computed evolutions for linear and nonlinear cases are shown in Figures 9(a)-(f) for the same values of time $t$ as used in Figures 8(a)-(f). For both linear $(f=0)$ and nonlinear $(f=1)$ cases the wave shape is preserved during evolution i.e. propagation and reflections. For nonlinear tensile stress wave, the material density reduces locally during deformation (due to elongation) which results in increasing local wave speed. Hence, in Figures 9(a)-(f) we observe that the nonlinear wave leads the linear wave throughout the evolution. The results for the evolution of velocity are not presented for brevity.

\subsubsection{Linear and Nonlinear Waves in TVE Solid Continua}

In this section, we consider linear and nonlinear waves in TVE solid continua. These solids have elasticity, mechanism of dissipation i.e. conversion of mechanical energy into entropy production which results in heat, hence influences specific internal energy. The dissipation mechanism is obviously present in linear (small strain) as well as nonlinear cases (Green's strain). For linear case, $(f=0)$, here also (as in the case of TE solid continua, Section 6.3.1) $\frac{\partial u}{\partial x} \ll 1$, hence $\rho_{0}=\rho(x, t)$ holds during evolution i.e. no change in density hence constant wave speed during evolution. In the case of TVE solid continua, we can take more liberty with the magnitude of stress $\sigma_{1}$ due to not being restricted by the instability issues. We consider dimensionless damping coefficient $c=0.006$ in all numerical studies presented in this section. 


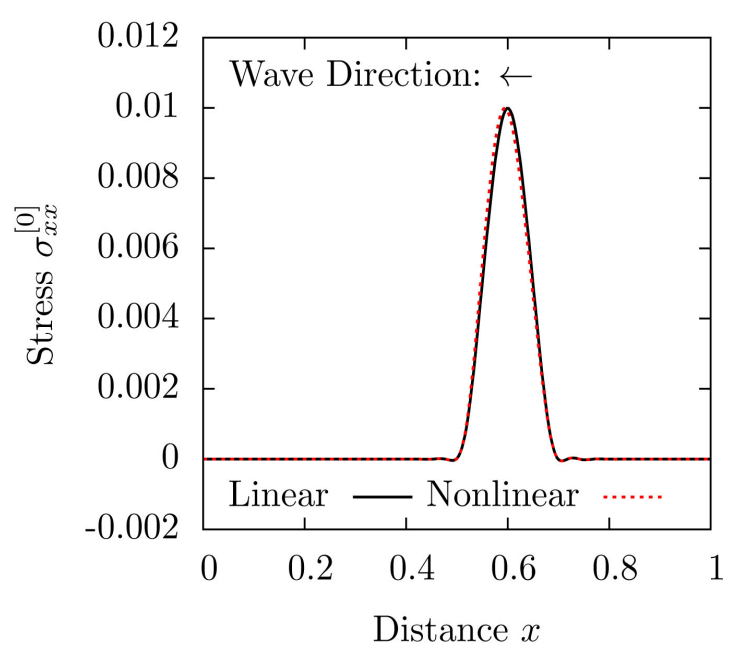

(a)

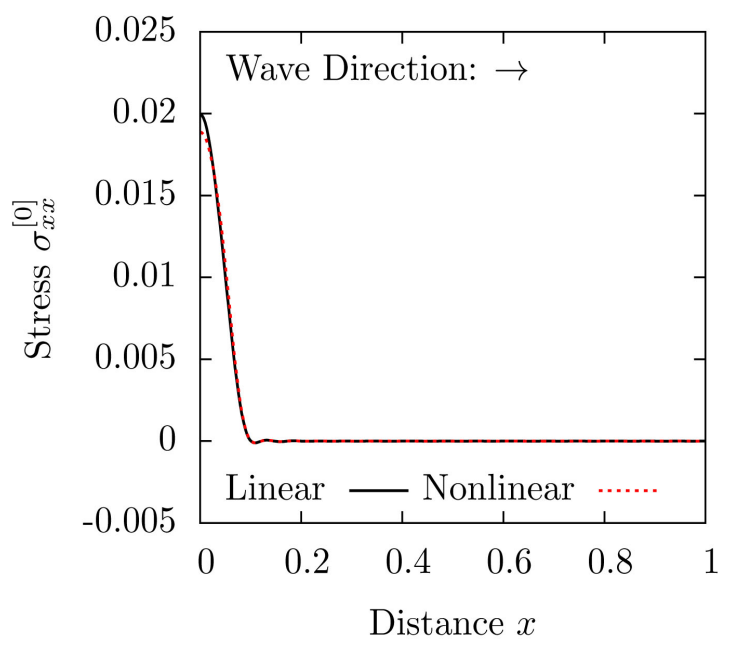

(c)

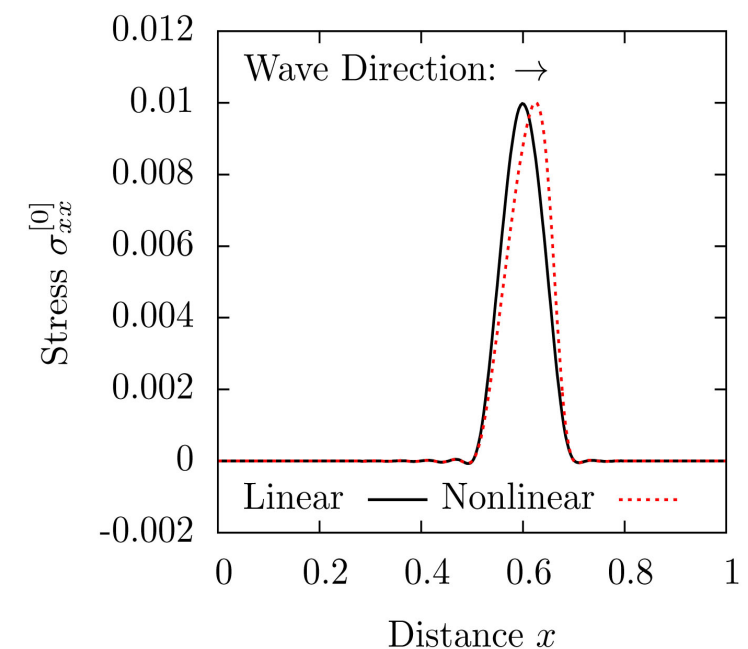

(e)

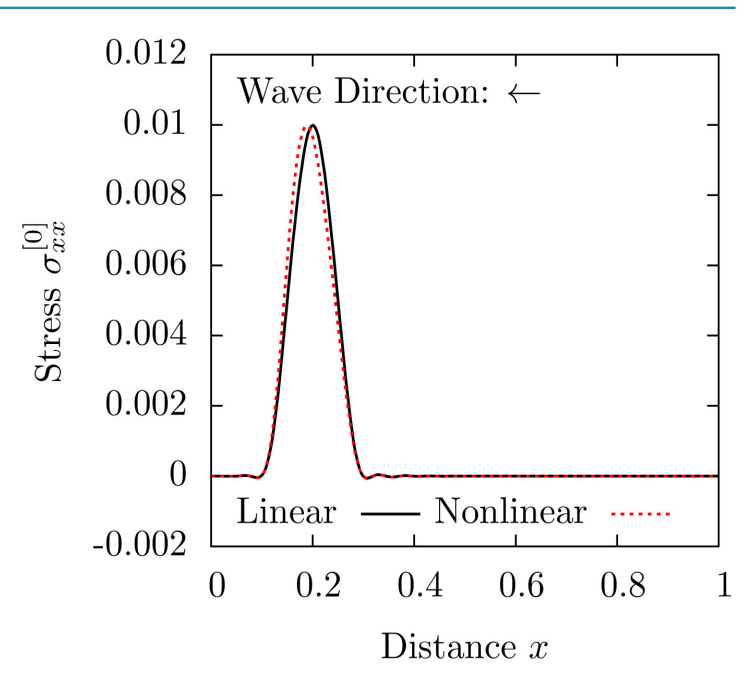

(b)

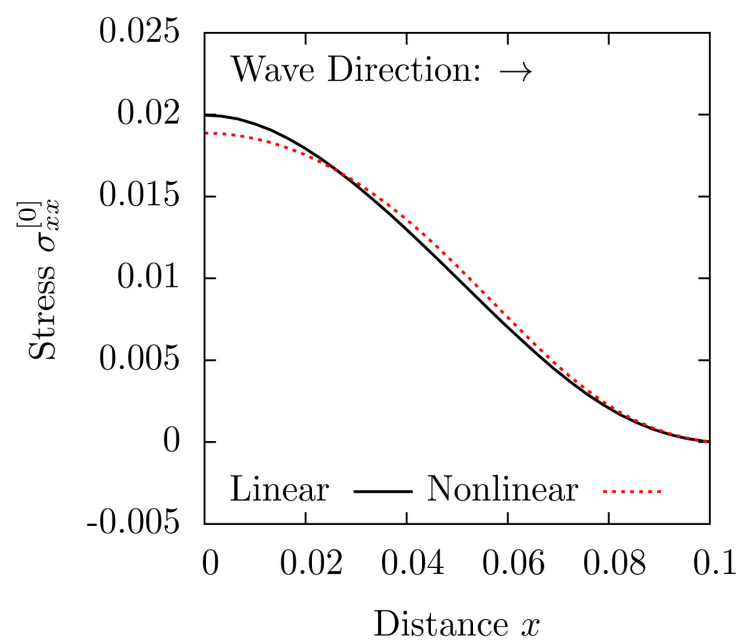

(d)

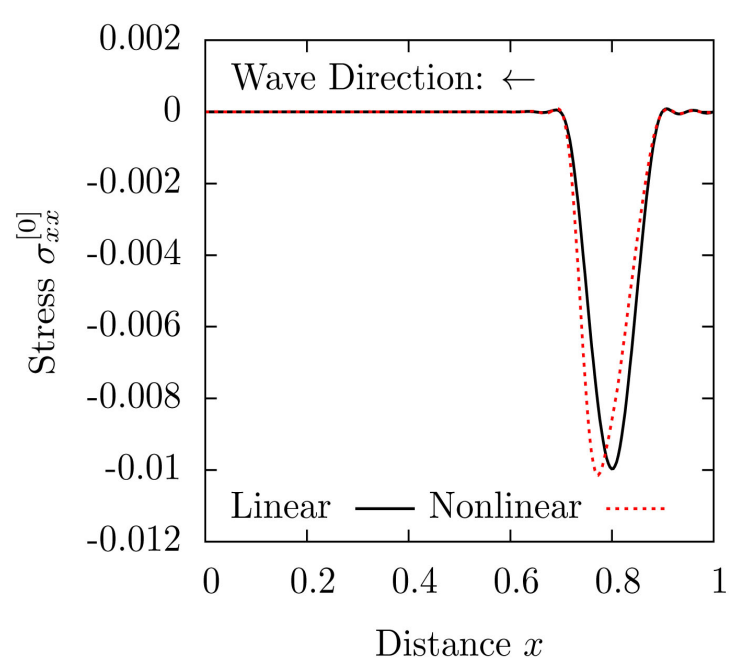

(f)

Figure 7. Evolution of $\sigma_{x x}^{[0]}$ along the length of the rod: TE, L1, $\Delta t=0.1, \sigma_{1}=-0.01$. (a) $t=5 \Delta t$; (b) $t=9 \Delta t$; (c) Reflection, $t=11 \Delta t$; (d) Details of reflection, $t=11 \Delta t$; (e) $t=17 \Delta t$; (f) $t=23 \Delta t$. 


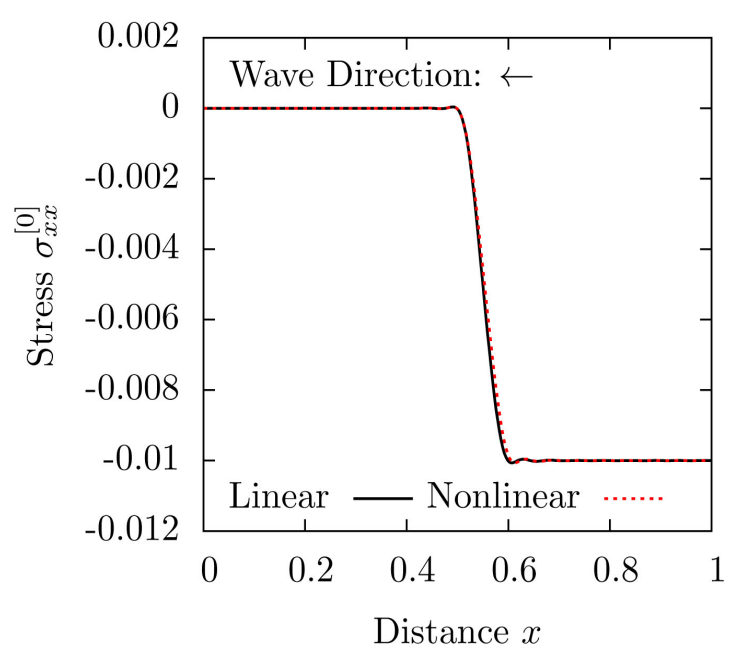

(a)

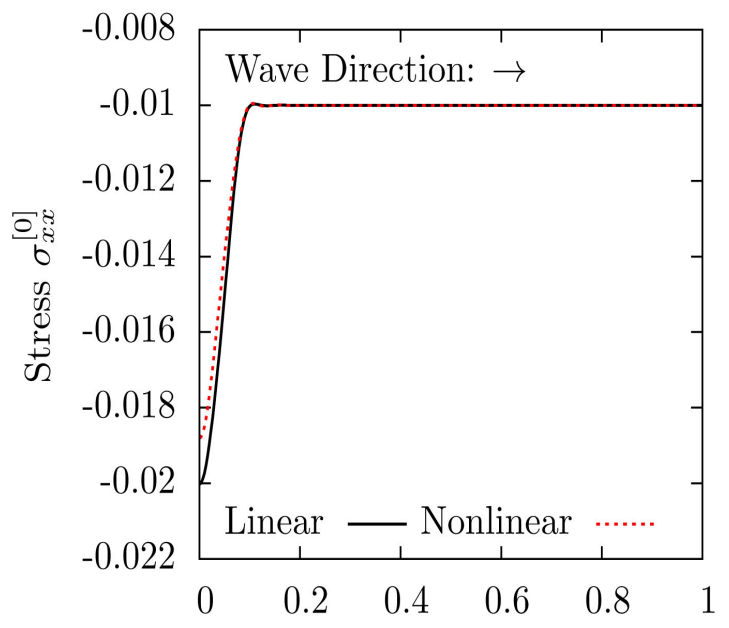

(c)

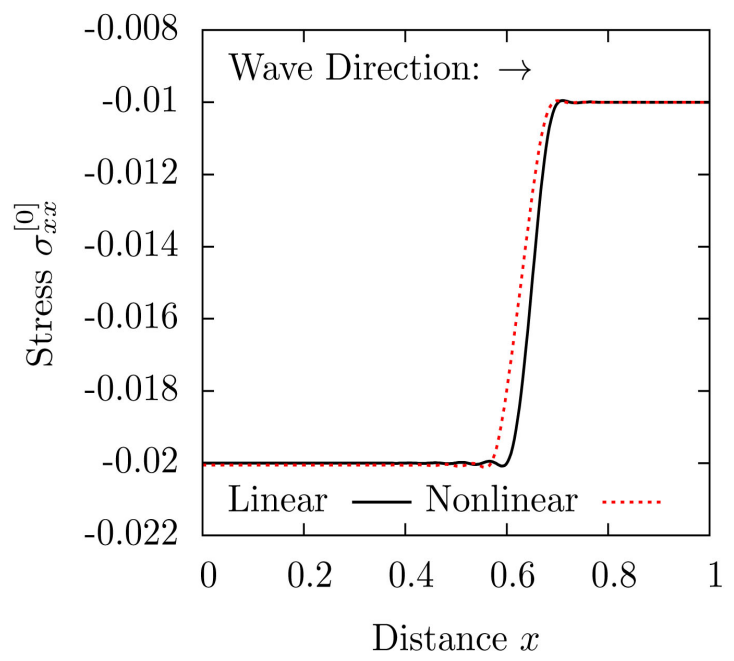

(e)

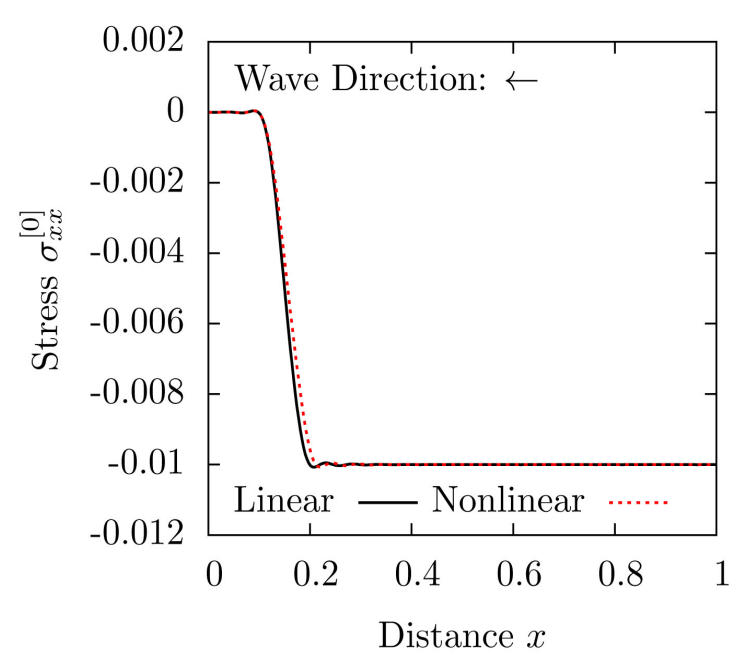

(b)

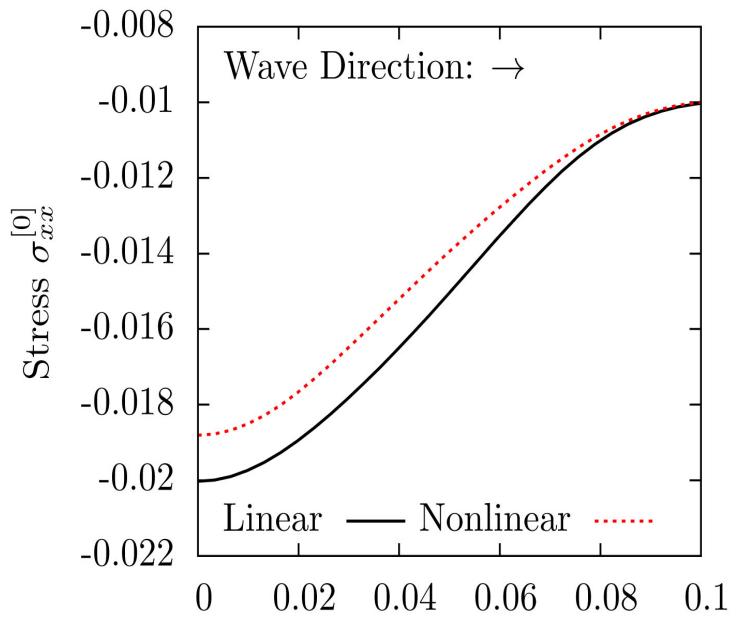

(d)

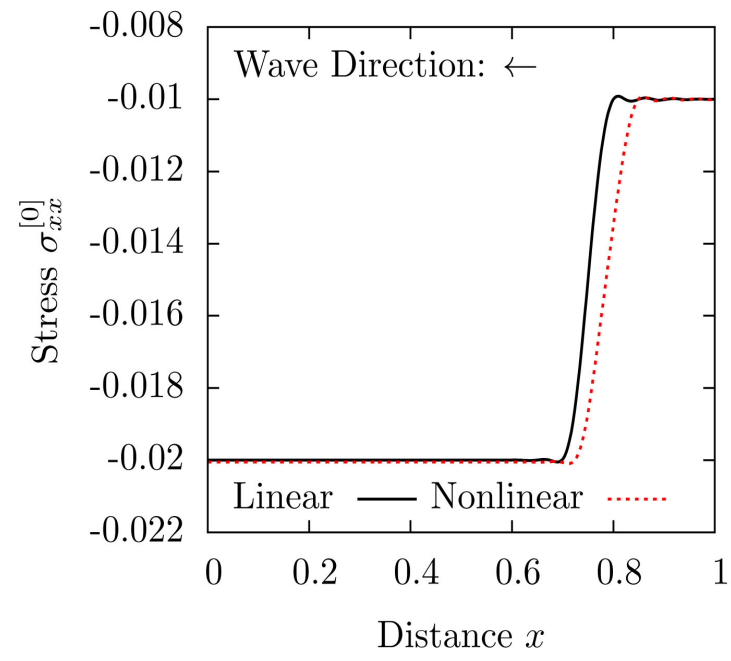

(f)

Figure 8. Evolution of $\sigma_{x x}^{[0]}$ along the length of the rod: TE, L2, $\Delta t=0.1, \sigma_{1}=-0.01$. (a) $\mathrm{t}=5 \Delta t$; (b) $t=9 \Delta t$; (c) Reflection, $t=11 \Delta t$; (d) Details of reflection, $t=11 \Delta t$; (e) $t=17 \Delta t$; (f) $t=23 \Delta t$. 


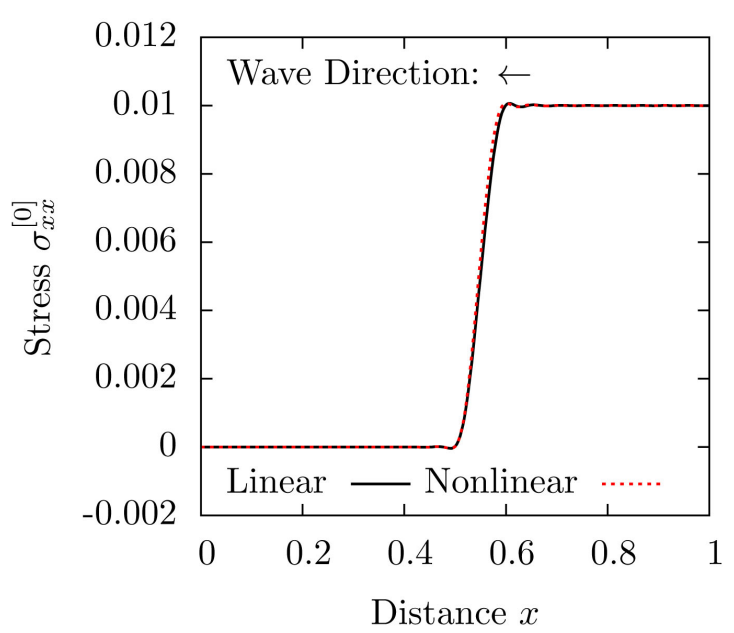

(a)

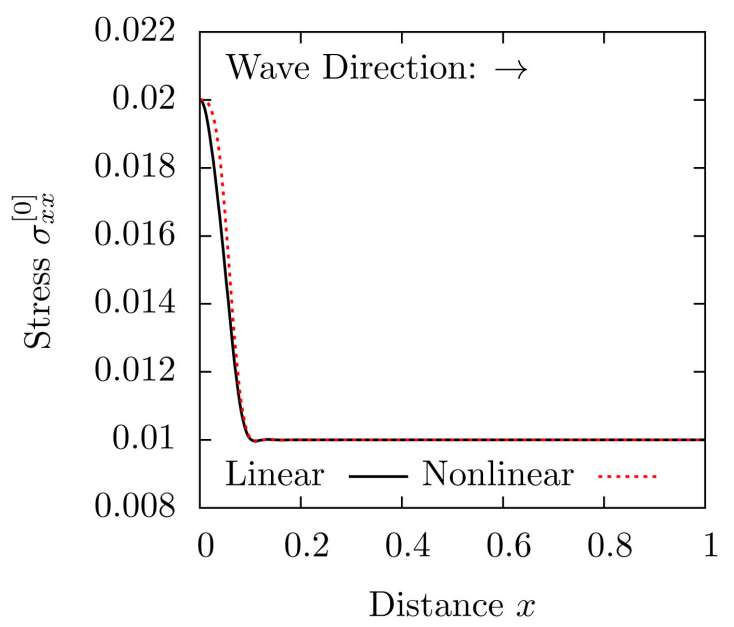

(c)

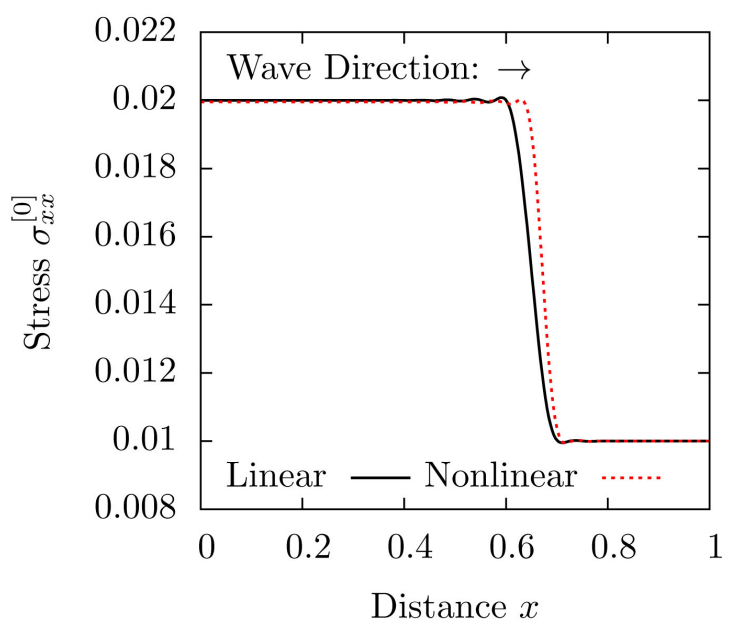

(e)

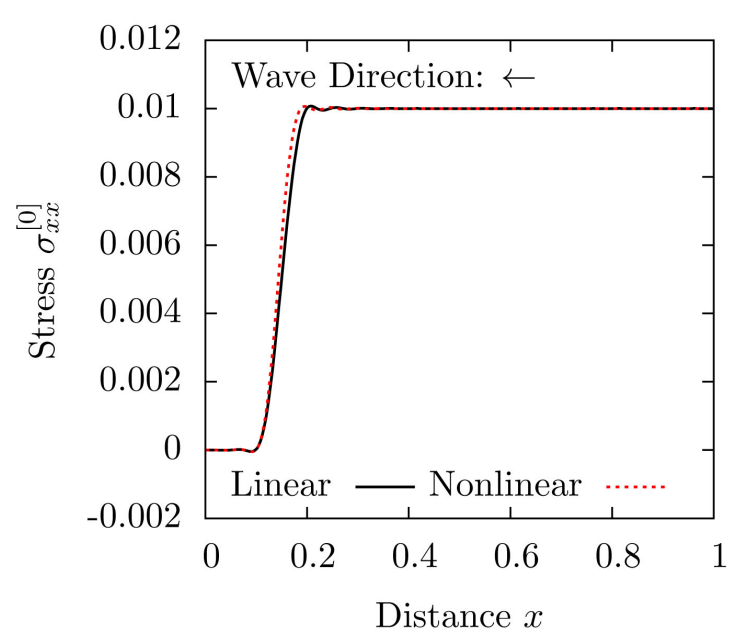

(b)

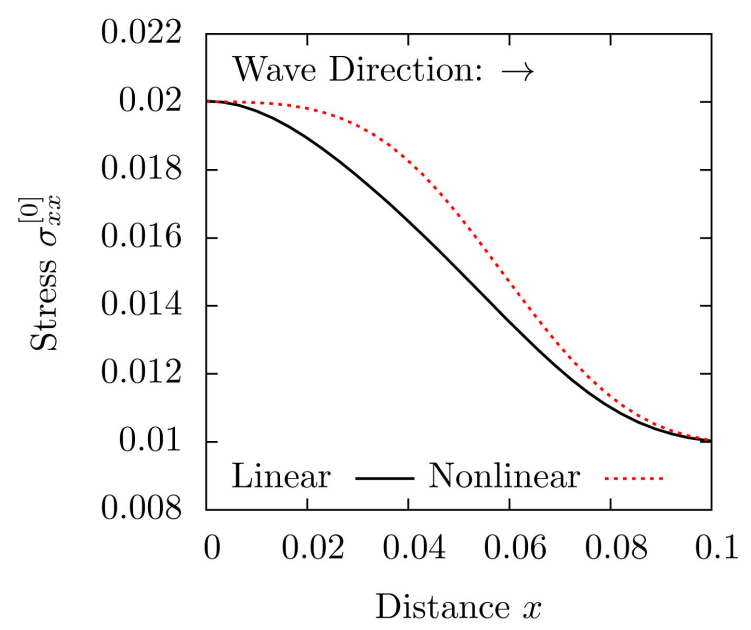

(d)

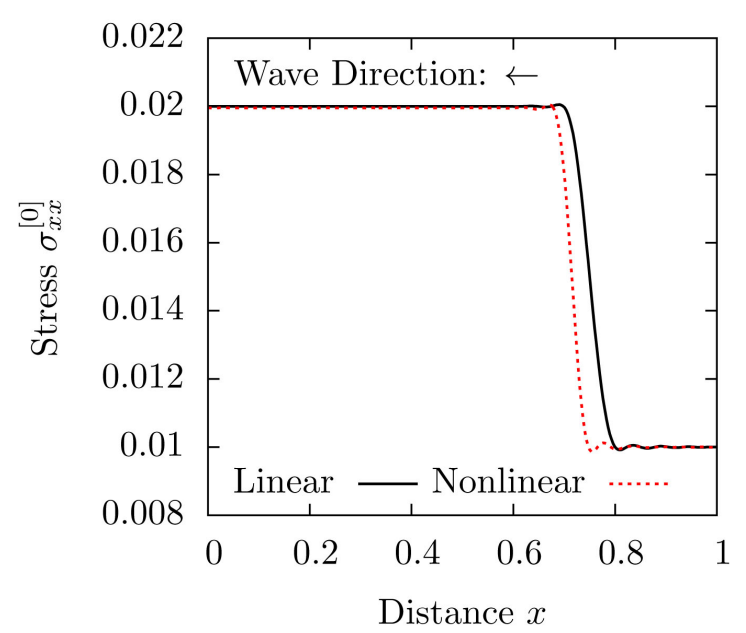

(f)

Figure 9. Evolution of $\sigma_{x x}^{[0]}$ along the length of the rod: TE, L2, $\Delta t=0.1, \sigma_{1}=-0.01$. (a) $t=5 \Delta t$; (b) $t=9 \Delta t$; (c) Reflection, $t=11 \Delta t$; (d) Details of reflection, $t=11 \Delta t$; (e) $t=17 \Delta t$; (f) $t=23 \Delta t$. 


\section{Loading L1}

\section{(a) Compressive}

Evolutions are computed for compressive pulse of $\sigma_{1}=-0.1$ over $0 \leq t \leq 2 \Delta t$. Figures 10(a)-(f) show evolutions of linear and nonlinear waves for $t=5 \Delta t, 9 \Delta t, 11 \Delta t, 17 \Delta t$, and $23 \Delta t$. In both linear and nonlinear waves, the amplitudes of the waves progressively decays and the support elongates as the evolution proceeds. At $t=17 \Delta t$ (Figure 10(e)) the peak value is only $40 \%$ of the peak of the original wave initiated at the commencement of the evolution. Due to local increase in density for the nonlinear case, the evolution for the nonlinear wave lags the evolution for the linear case. Nonlinear wave evolution consistently exhibits lower peak stress values compared to linear case. Since in TVE solid continua, there is entropy production due to rate of mechanical work, hence heat generation due to mechanical work, this would result in temperature changes along the length of the rod during evolution. In the studies conducted here, the initial dimensionless temperature at time $t=0$ is considered to be 1 i.e. $\theta=1$ is used as initial condition. Figures 11(a)-(f) show temperature distributions along the rod for the same values of time as in Figure 10. Figure 11(d) is an exploded view of Figure 11(c). We observe that the nonlinear case lags the linear case, lower peak values for nonlinear case and quite complex temperature distribution along $0 \leq x \leq 1$ after wave reflection from $x=0.0$ boundary (Figure 11(e) and Figure 11(f)).

(b) Tensile

When $\sigma_{1}=0.1$ for loading L1, we have a tensile pulse. Computed evolutions for same values of time $t$ as in the case of compressive loading are shown in Figures 12(a)-(f). Due to dissipation, the wave peaks are reduced for both linear and nonlinear cases. The nonlinear wave peak values are slightly higher than those of the corresponding linear waves. Whereas in the case of compression, the peaks of linear waves are higher than those of nonlinear waves. The evolution of linear waves lags the evolution of nonlinear waves due to a decrease in density (because of tension), hence increased wave speed in the locally deformed region of $0 \leq x \leq 1$ occupied by the wave. Reflection of the wave at $x=0.0$ (Figure 12(c) and Figure 12(d) at $t=11 \Delta t$ ) and from the $x=1.0$ boundary (Figure 12(f) at $t=23 \Delta t$ ) are smooth and present no problems. Evolution of temperature is shown in Figures 13(a)-(f). Evolution of temperature for the nonlinear wave leads the linear wave. This is consistent with the evolution of stress wave in Figures 12(a)-(f). Overall, we observe higher temperature peaks in this case compared to compressive wave. Complex temperature distribution in Figure 13(e) and Figure 13(f) after reflection are simulated accurately ( $I$ of the order of $O\left(10^{-13}\right)$ or lower for each space-time strip).

Loading L2: Tensile

In this case we consider tensile ramp loading with $\sigma_{1}=0.4$. We consider such high values of $\sigma_{1}$ to demonstrate more clearly the shock formation in case of nonlinear waves. In tension, such high values of $\sigma_{1}$ can be used as in tension we do not have the problem of instability. Dimensionless damping coefficient $c$ is chosen to be 0.006 , same as in loading L1. Because of high value of $\sigma_{1}$, large elongation and significant progressive reduction in density will occur. This results in substantial and progressively increased wave speed. As a consequence, the waves behind the waves are moving at faster speeds resulting in "piling up" of the waves which ultimately results in a sharp front referred to as a shock. Figures 14(a)-(f) show evolution of stress for both linear

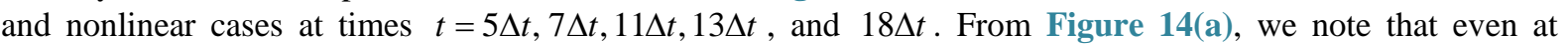
$t=5 \Delta t$, the nonlinear wave has steepened significantly compared to linear wave confirming shock formation. Comparing evolutions of the linear and the nonlinear waves in Figure 14(a) and Figure 14(b) for $t=5 \Delta t$ and at $t=7 \Delta t$, we note that between time $t=5 \Delta t$ to time $t=7 \Delta t$, the right portion of the wave is travelling faster than the lower left portion of the wave resulting in further steepening of the nonlinear wave in Figure 14(b). Reflection in Figure 14(c) and Figure 14(d) are smooth and present no problem. The nonlinear waves are travelling much faster than the linear waves, hence the nonlinear waves are always ahead of the linear waves throughout the evolution. This is dramatically illustrated in Figure 14(e) and Figure 14(f). The evolution of the temperature for the same time values as in Figures 14(a)-(f) is shown in Figures 15(a)-(f). Shock formation in the temperature evolution and its speed of propagation are similar to the stress wave evolutions shown in Figures 14(a)-(f). Due to the nature of the applied stress wave (ramp), the influence of dissipation can only be observed in the temperature evolution and not the stress evolution. Without dissipation, there would have been no change in temperature along the length of the rod.

\subsubsection{Linear and Nonlinear Waves in TVEM \\ Loading L1: Compressive and Tensile}




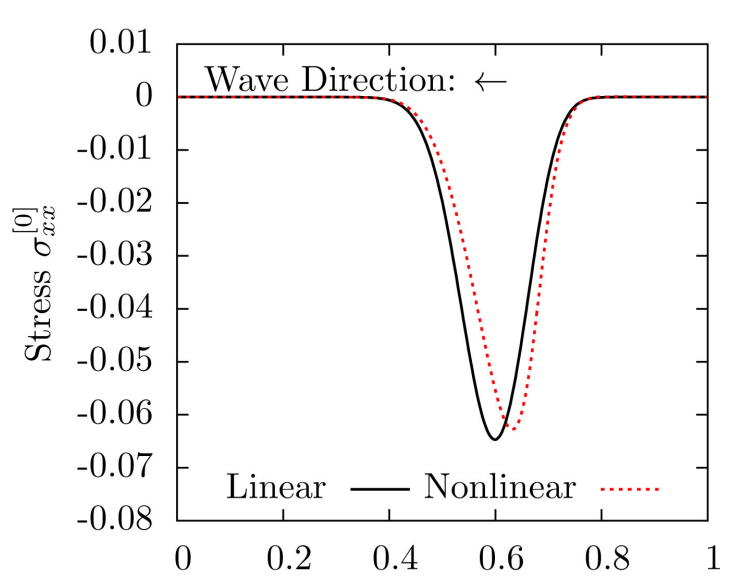

Distance $x$

(a)

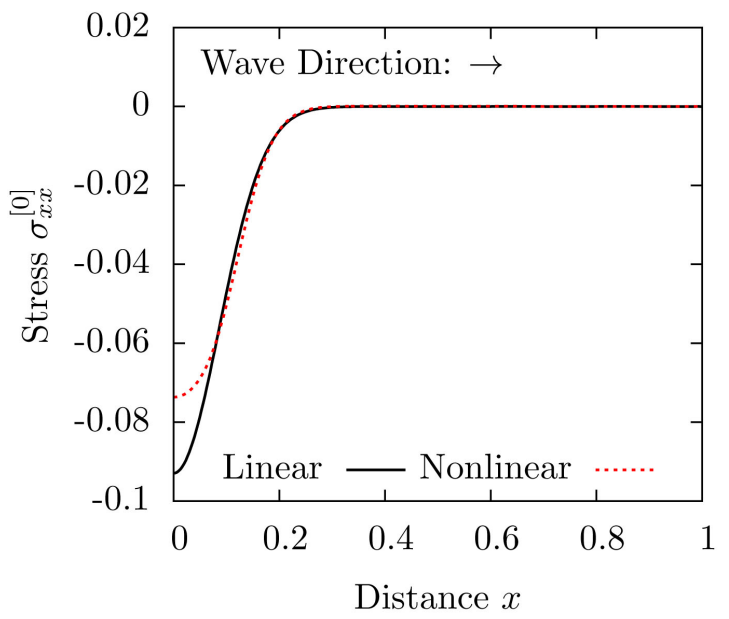

(c)

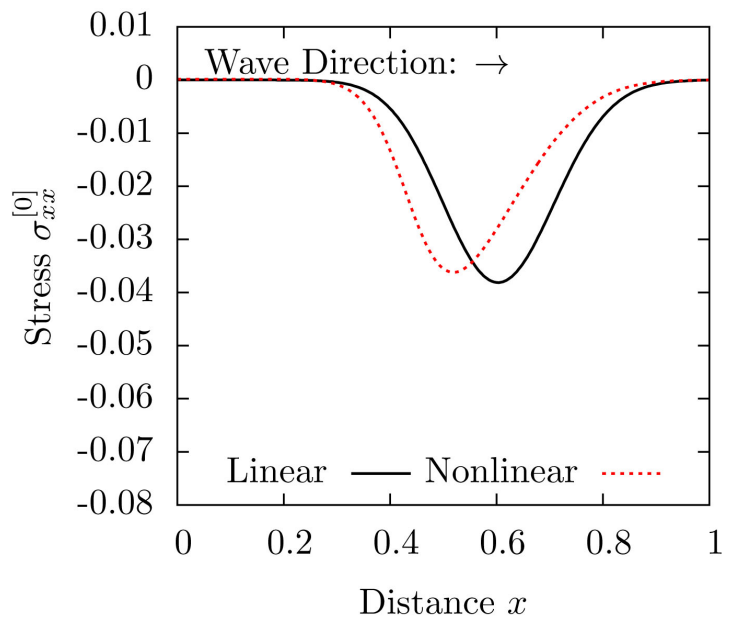

(e)

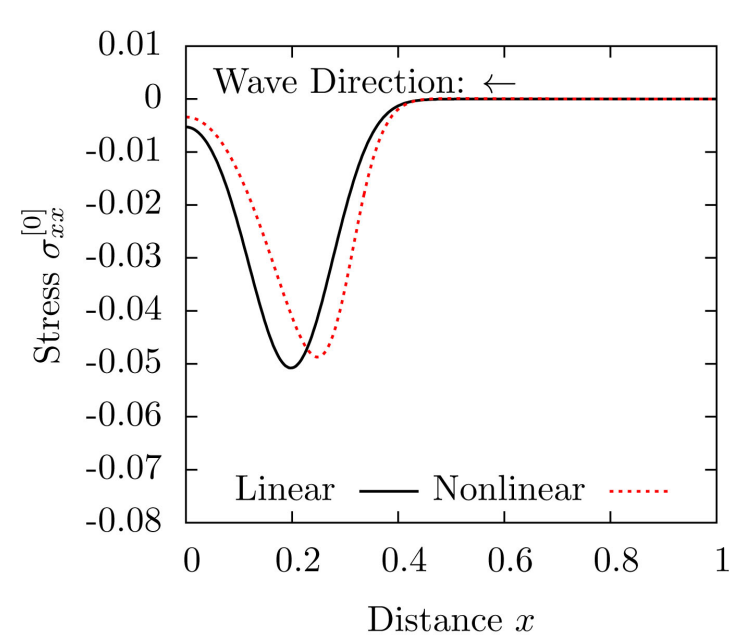

(b)

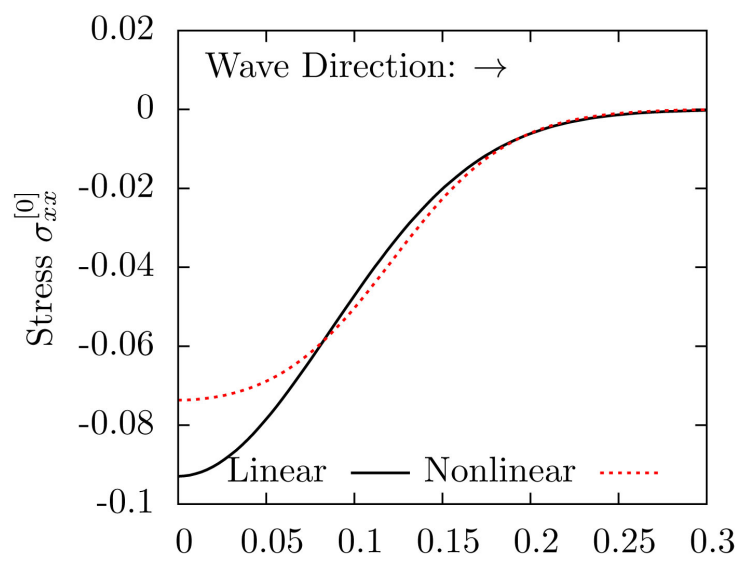

Distance $x$

(d)

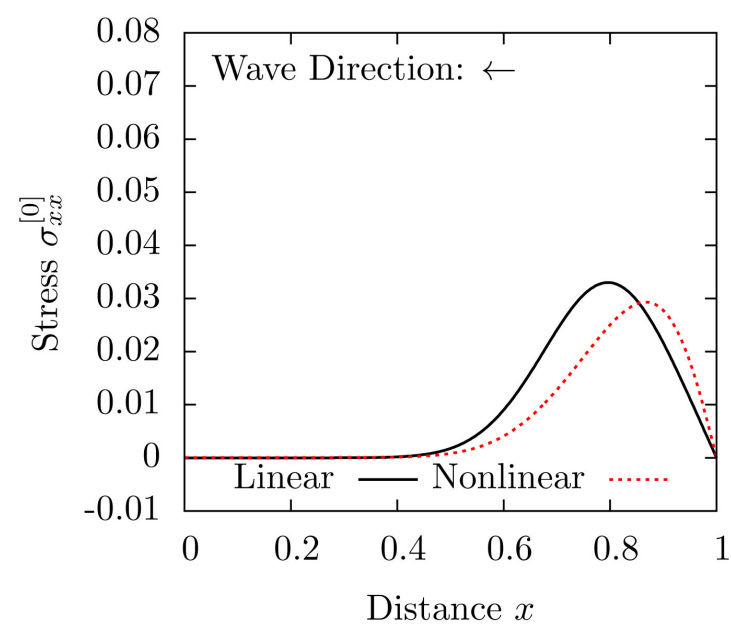

(f)

Figure 10. Evolution of $\sigma_{x x}^{[0]}$ along the length of the rod: TVE, L1, $\Delta t=0.1, \sigma_{1}=-0.1$. (a) $t=5 \Delta t$; (b) $t=9 \Delta t$; (c) Reflection, $t=11 \Delta t$; (d) Details of reflection, $t=11 \Delta t$; (e) $t=17 \Delta t$; (f) $t=23 \Delta t$. 


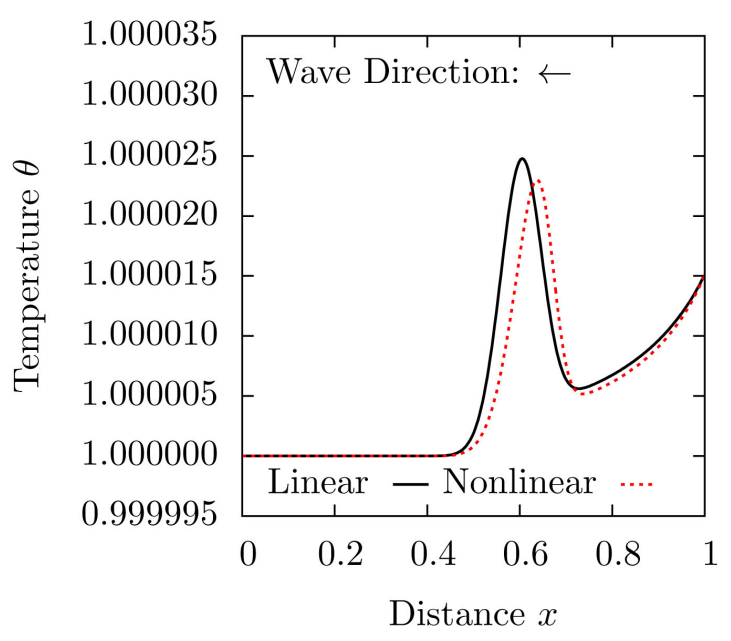

(a)

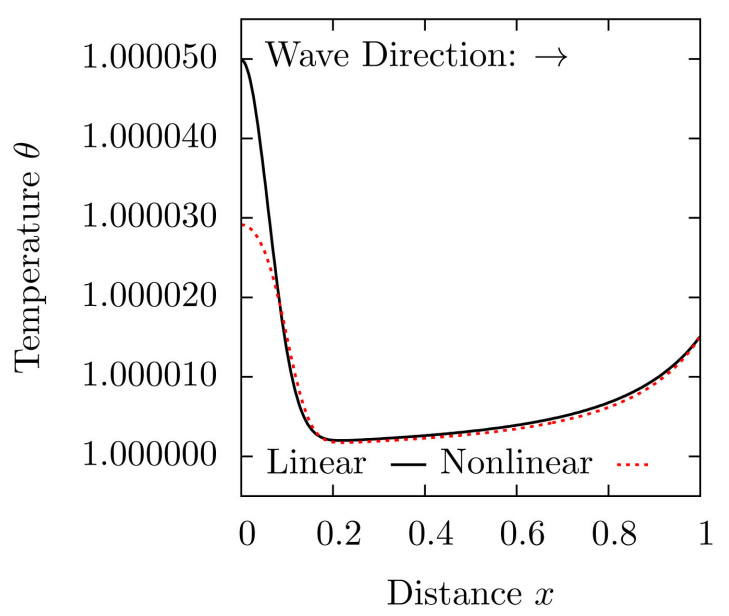

(c)

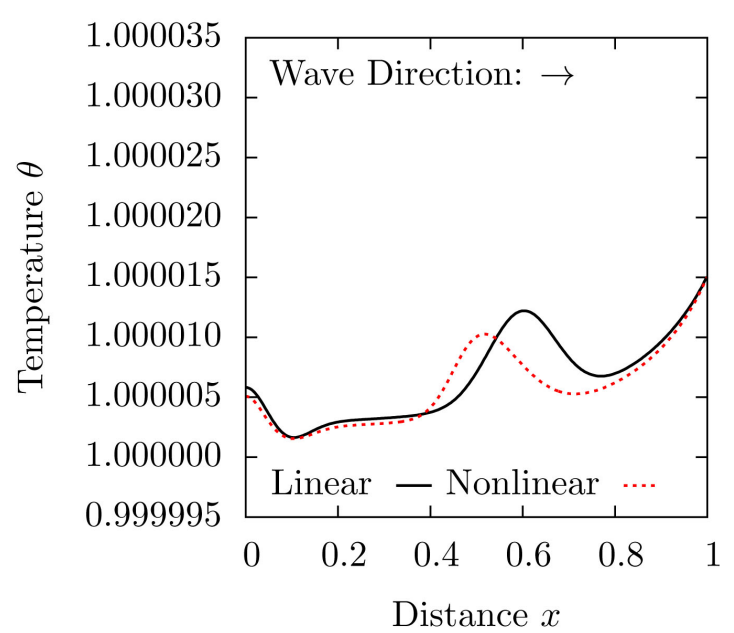

(e)

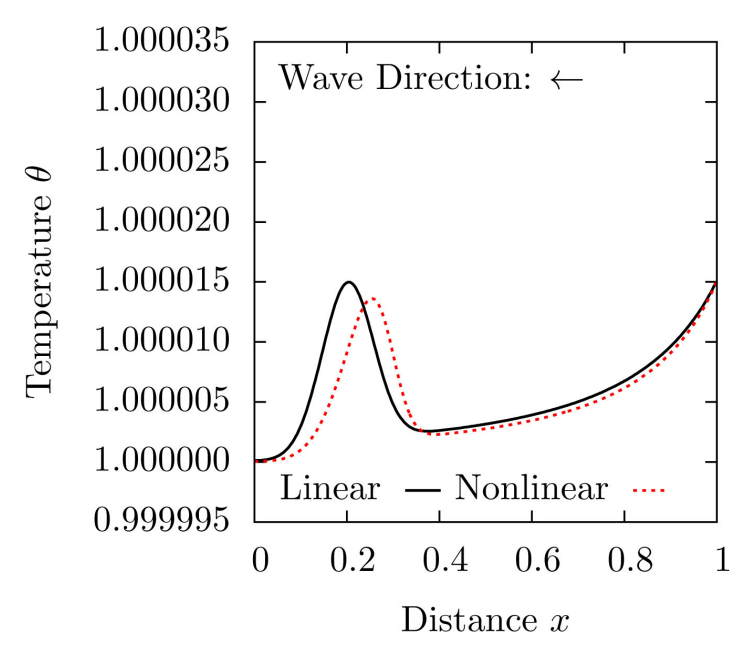

(b)

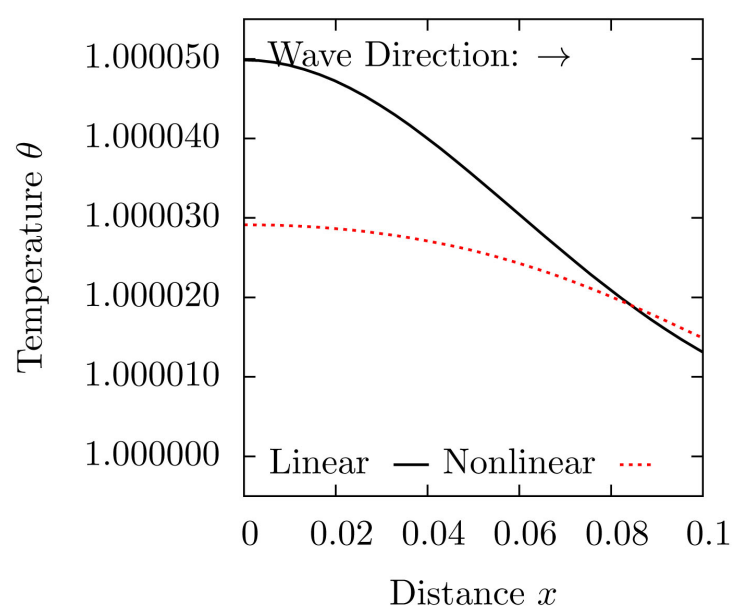

(d)

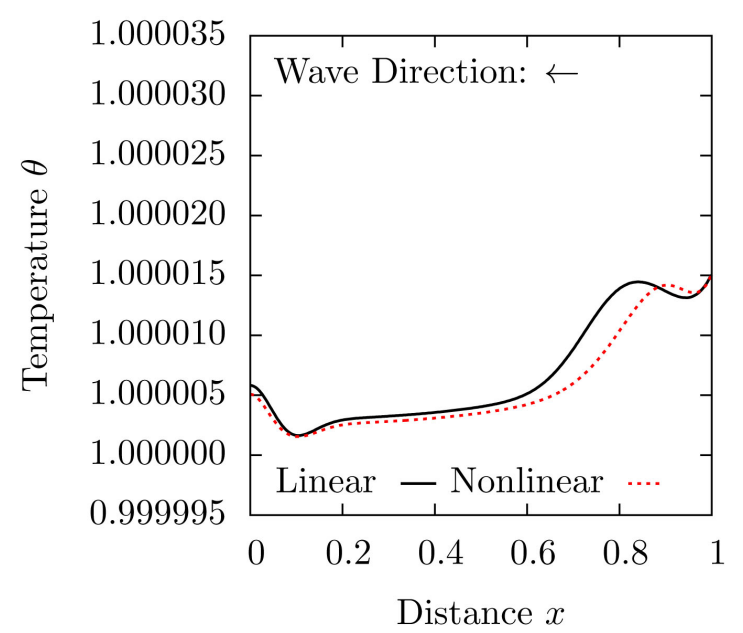

(f)

Figure 11. Evolution of temperature $\theta$ along the length of the rod: TVE, L1, $\Delta t=0.1, \sigma_{1}=-0.1$. (a) $t=5 \Delta t$; (b) $t=9 \Delta t$; (c) Reflection, $t=11 \Delta t$; (d) Details of reflection, $t=11 \Delta t$; (e) $t=17 \Delta t$; (f) $t=23 \Delta t$. 


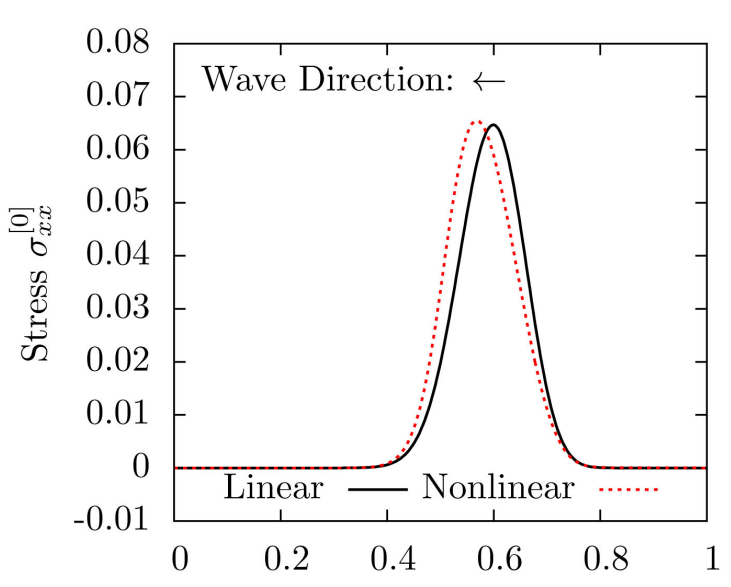

Distance $x$

(a)

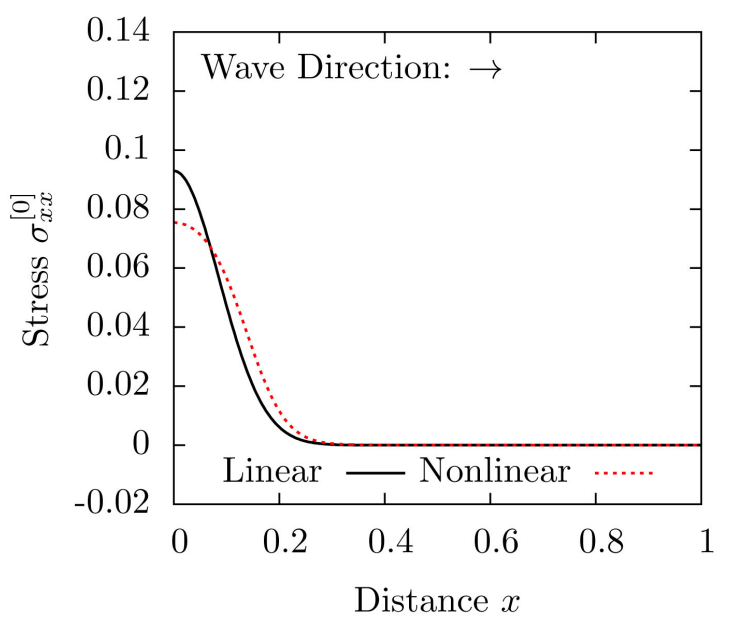

(c)

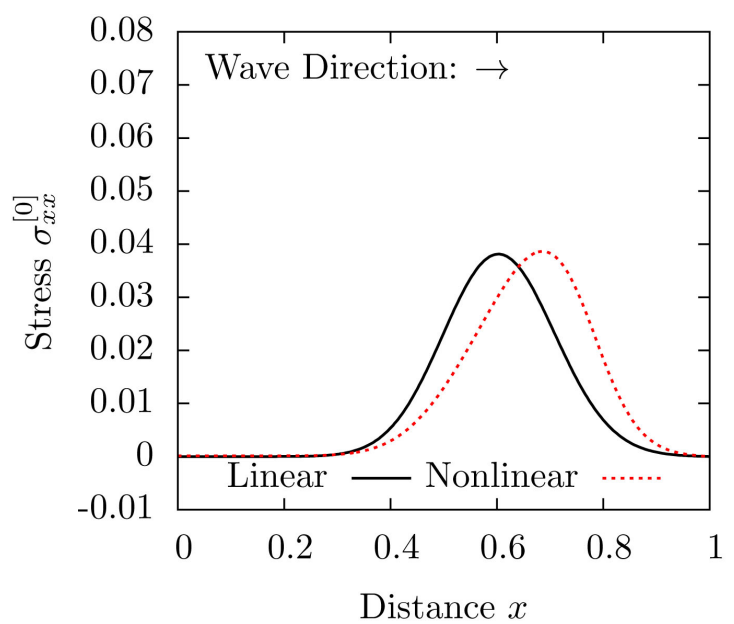

(e)

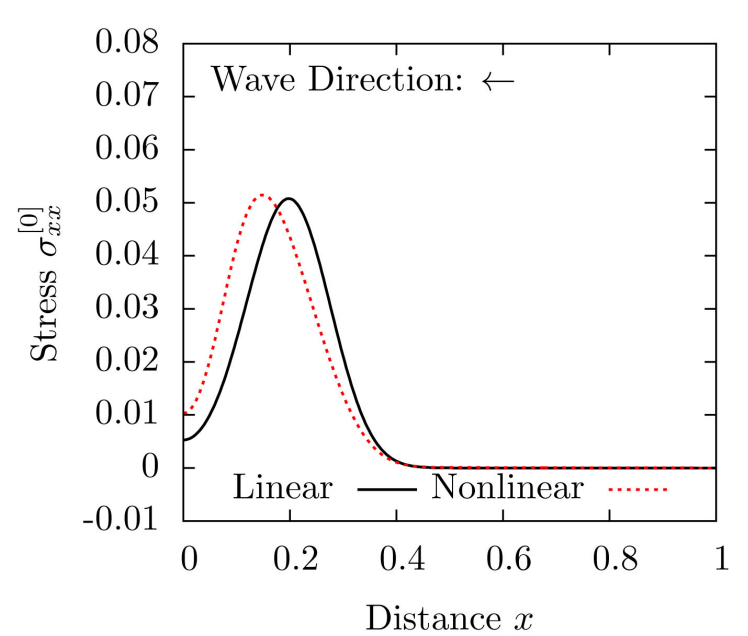

(b)

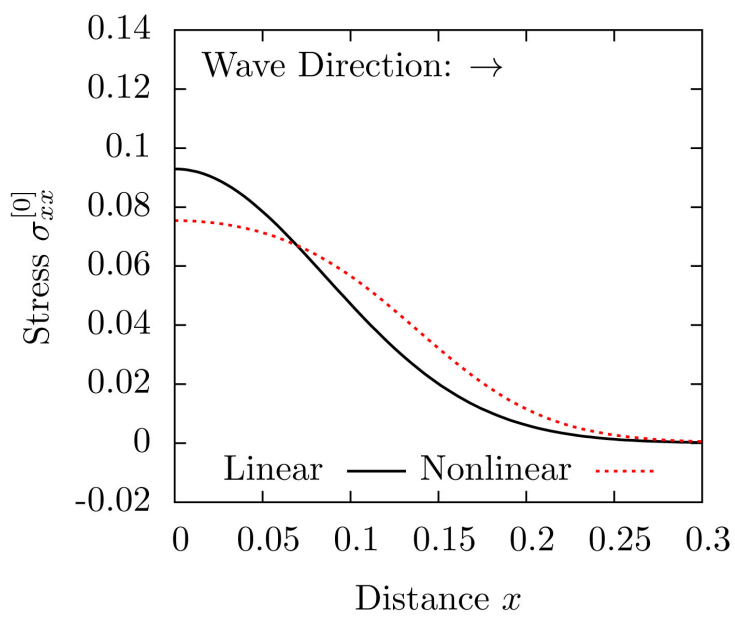

(d)

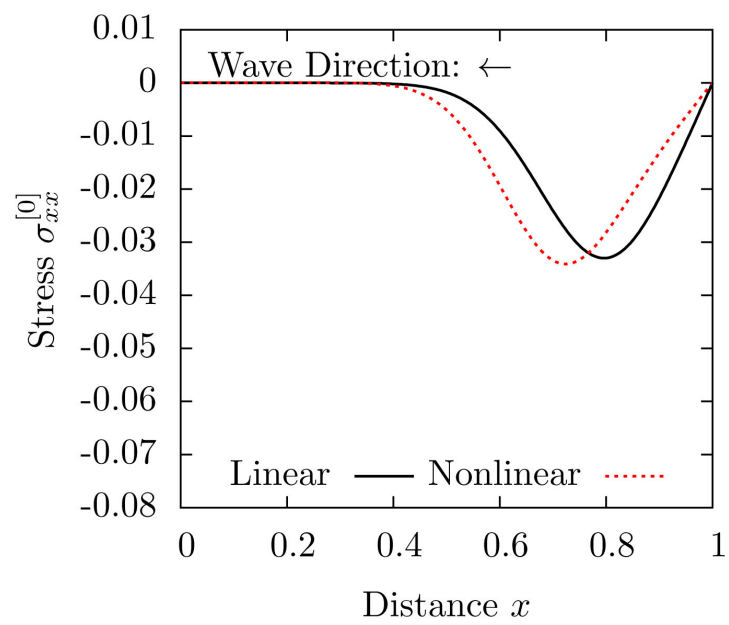

(f)

Figure 12. Evolution of $\sigma_{x x}^{[0]}$ along the length of the rod: TVE, L1, $\Delta t=0.1, \sigma_{1}=0.1$. (a) $t=5 \Delta t$; (b) $t=9 \Delta t$; (c) Reflection, $t=11 \Delta t$; (d) Details of reflection, $t=11 \Delta t$; (e) $t=17 \Delta t$; (f) $t=23 \Delta t$. 


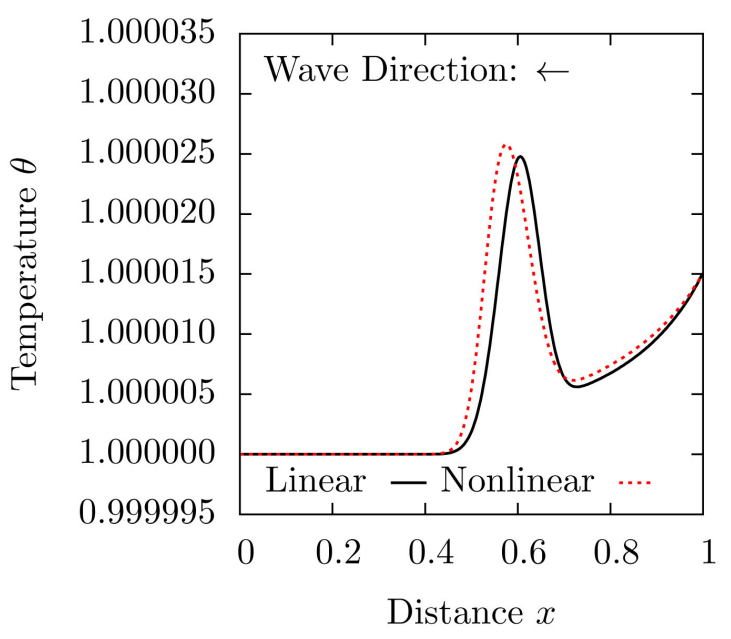

(a)

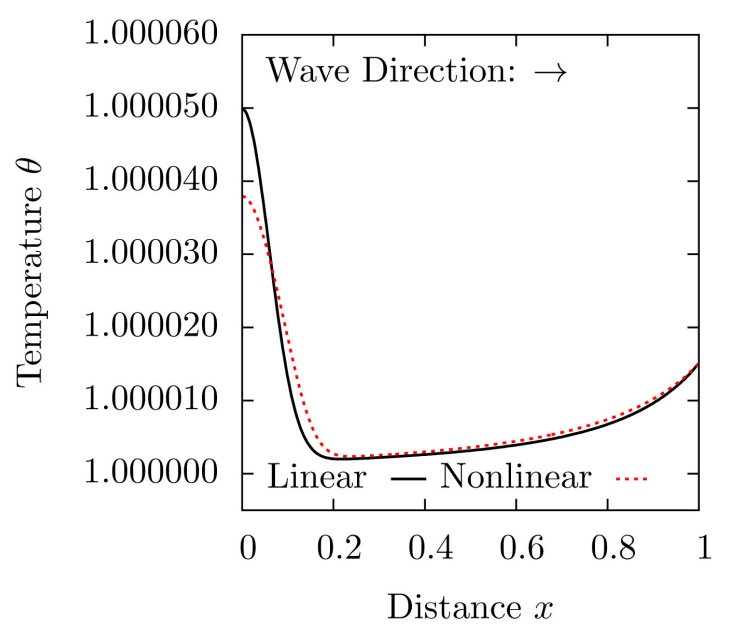

(c)

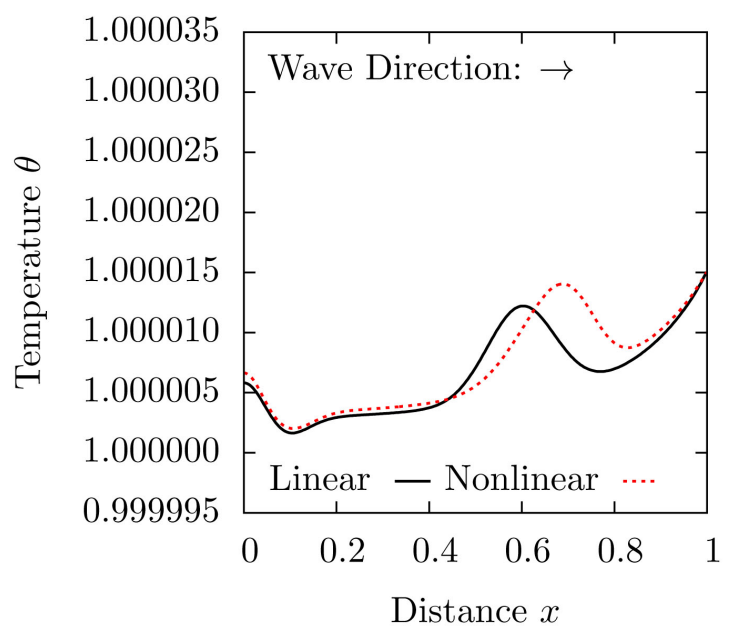

(e)

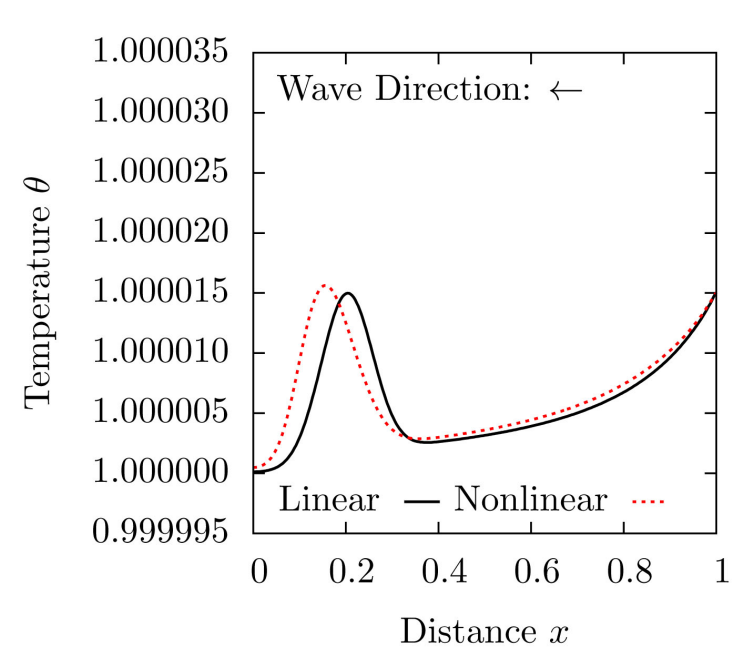

(b)

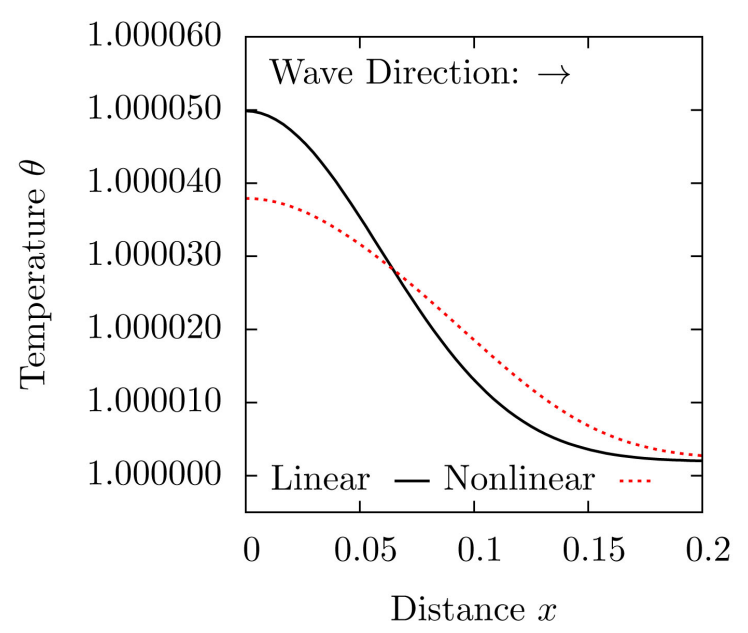

(d)

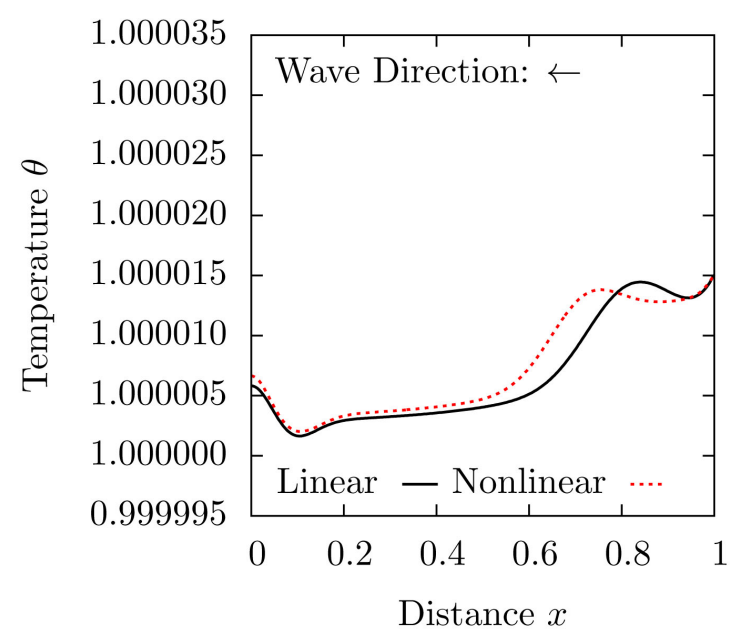

(f)

Figure 13. Evolution of temperature $\theta$ along the length of the rod: TVE, L1, $\Delta t=0.1, \sigma_{1}=0.1$. (a) $t=5 \Delta t$; (b) $t=9 \Delta t$; (c) Reflection, $t=11 \Delta t$; (d) Details of reflection, $t=11 \Delta t$; (e) $t=17 \Delta t$; (f) $t=23 \Delta t$. 


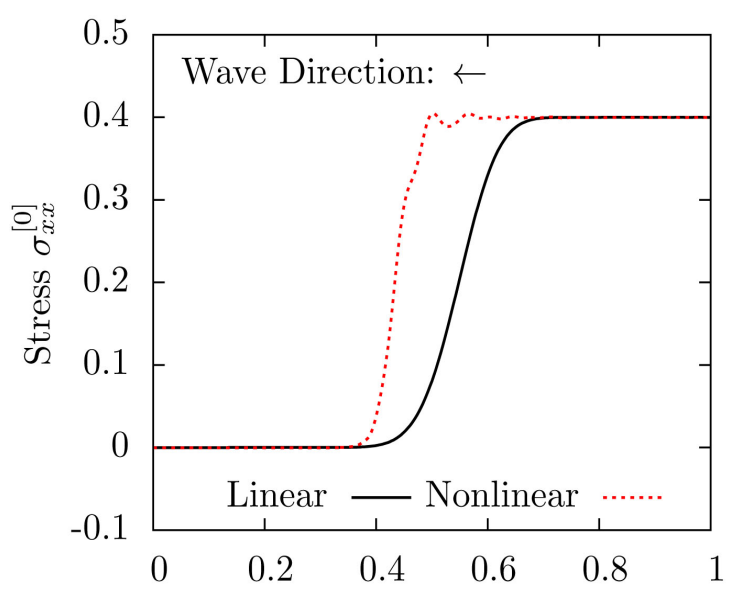

Distance $x$

(a)

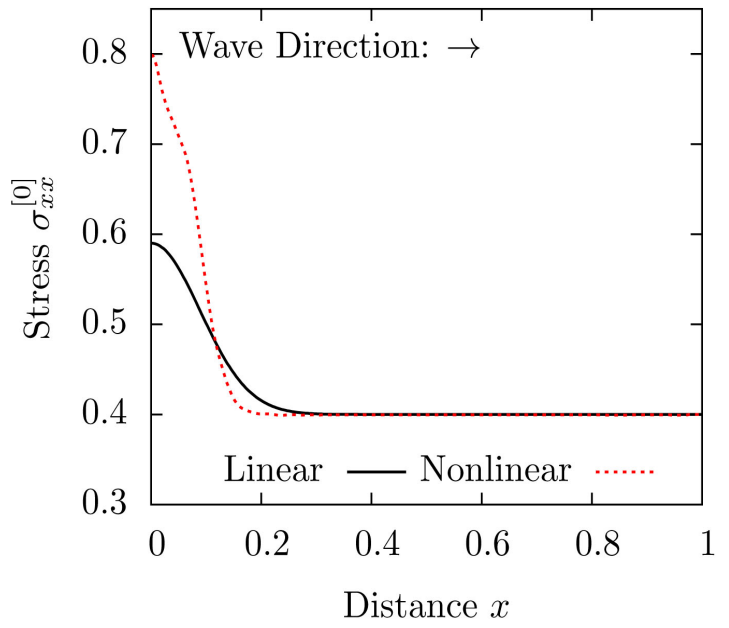

(c)

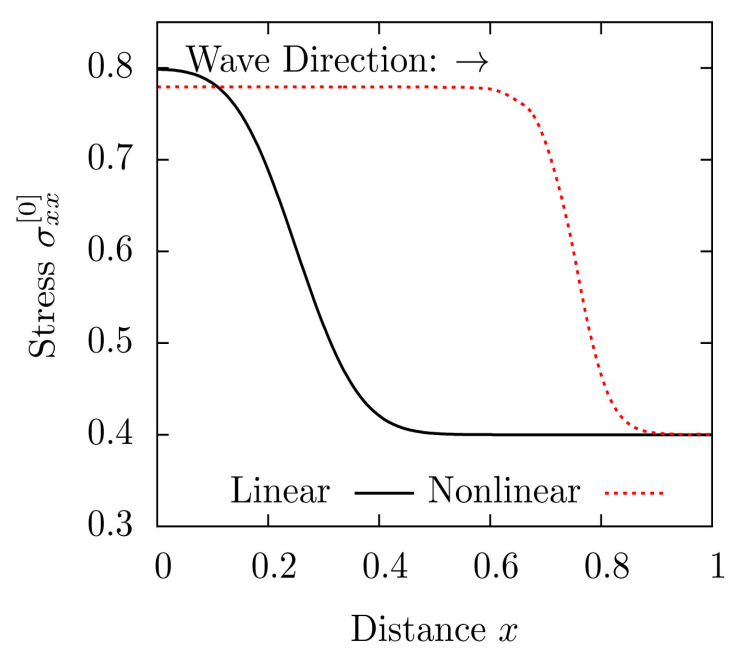

(e)

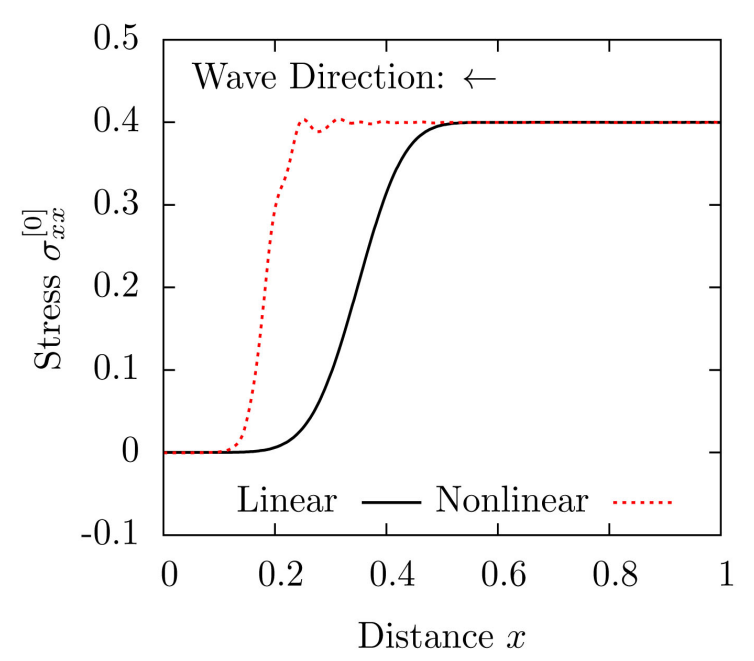

(b)

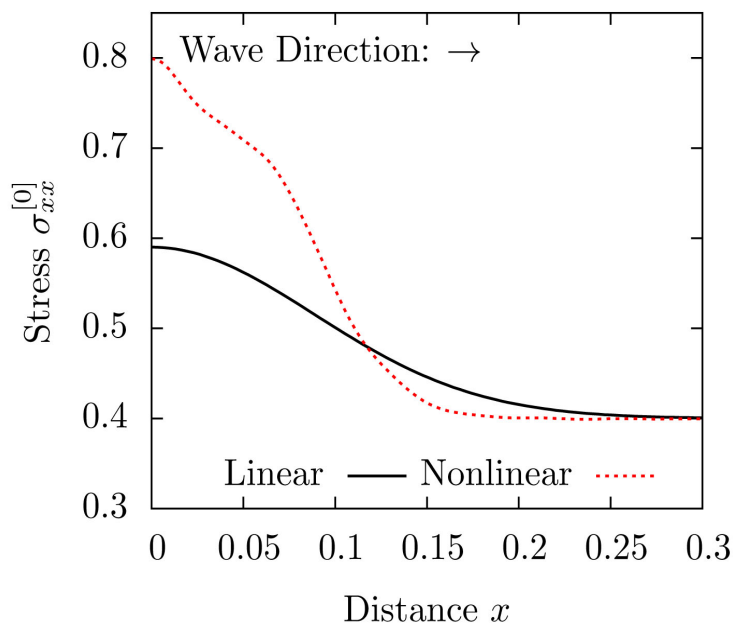

(d)

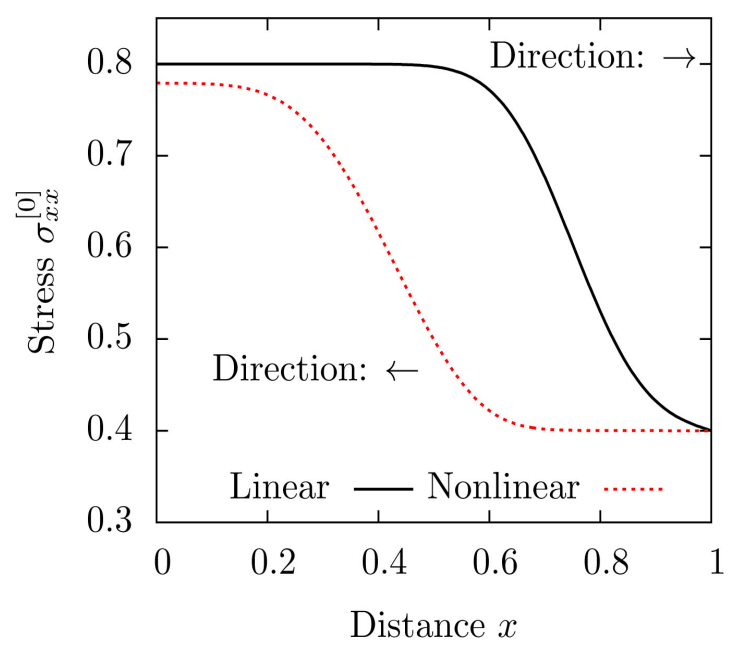

(f)

Figure 14. Evolution of $\sigma_{x x}^{[0]}$ along the length of the rod: TVE, L2, $\Delta t=0.1, \sigma_{1}=0.4$. (a) $t=5 \Delta t$; (b) $t=9 \Delta t$; (c) Reflection, $t=11 \Delta t$; (d) Details of reflection, $t=11 \Delta t$; (e) $t=17 \Delta t$; (f) $t=23 \Delta t$. 


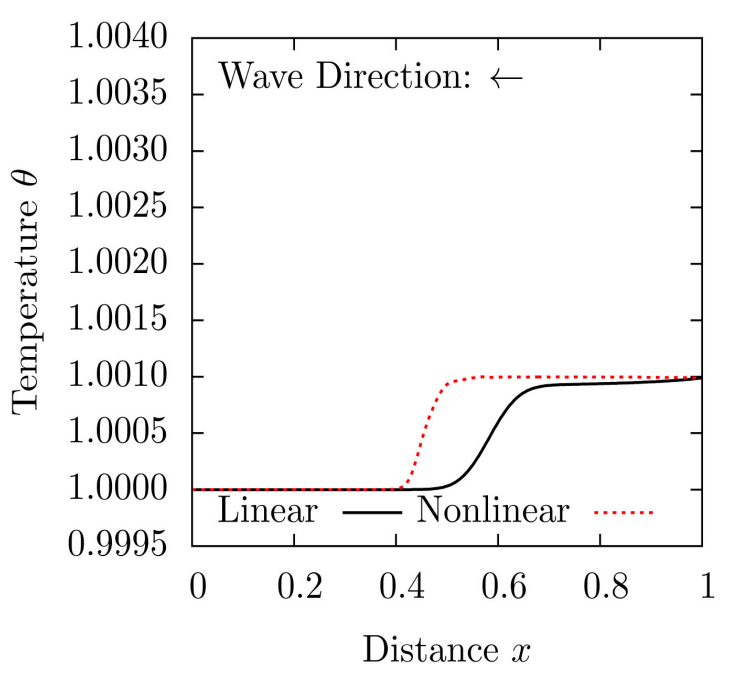

(a)

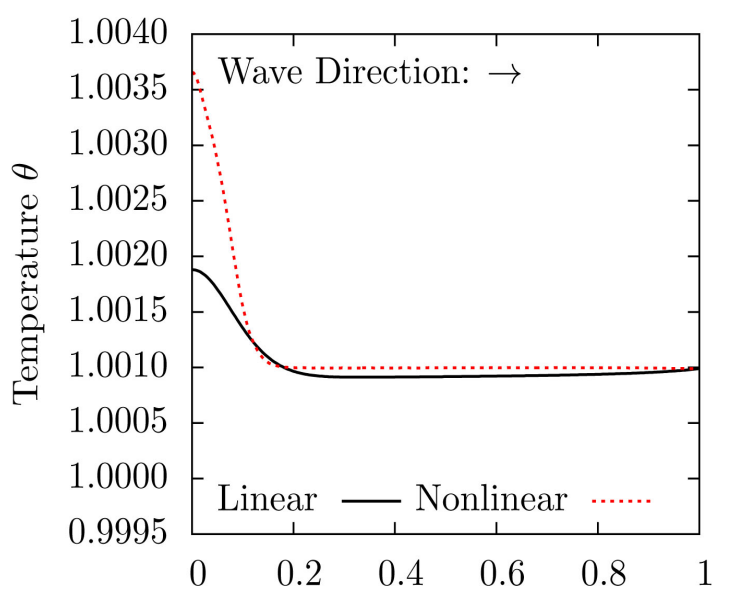

(c)

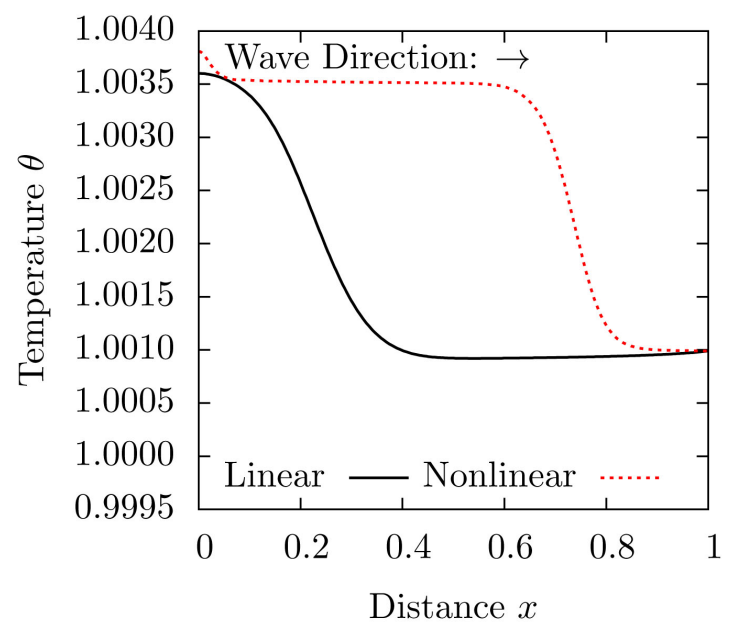

(e)

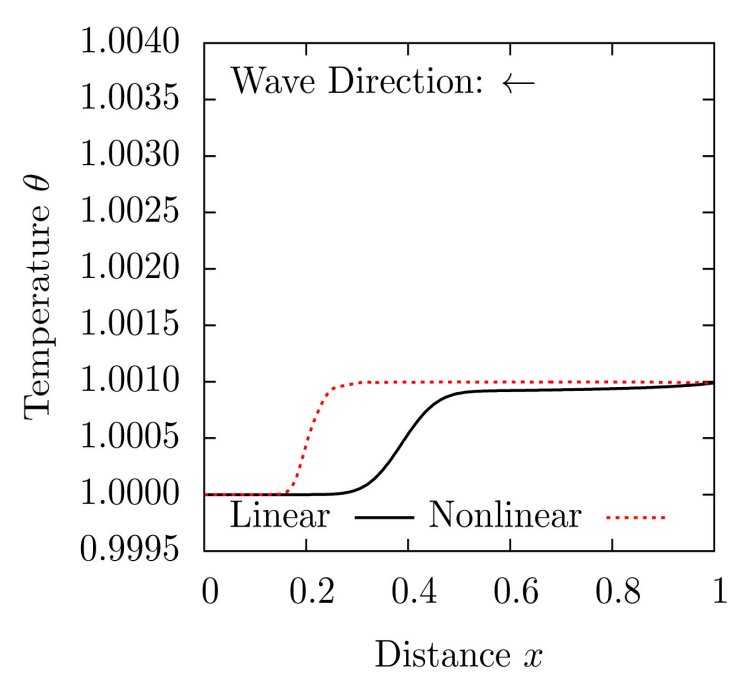

(b)

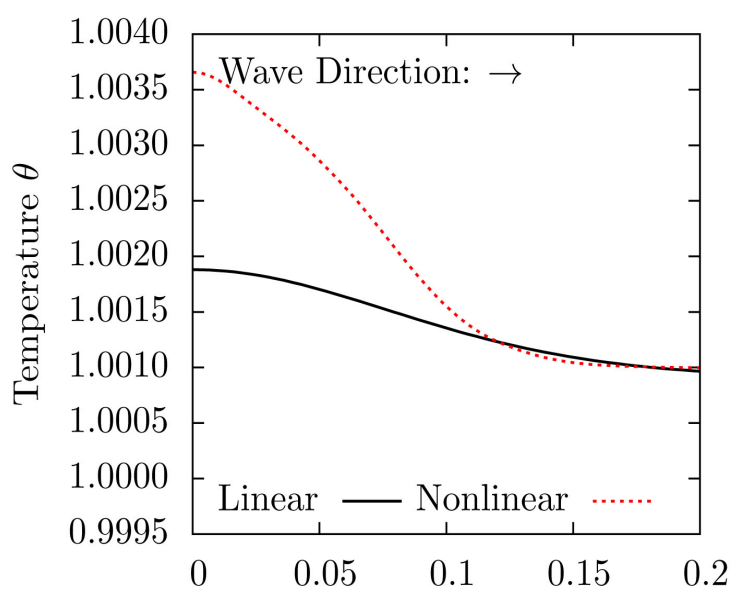

(d)

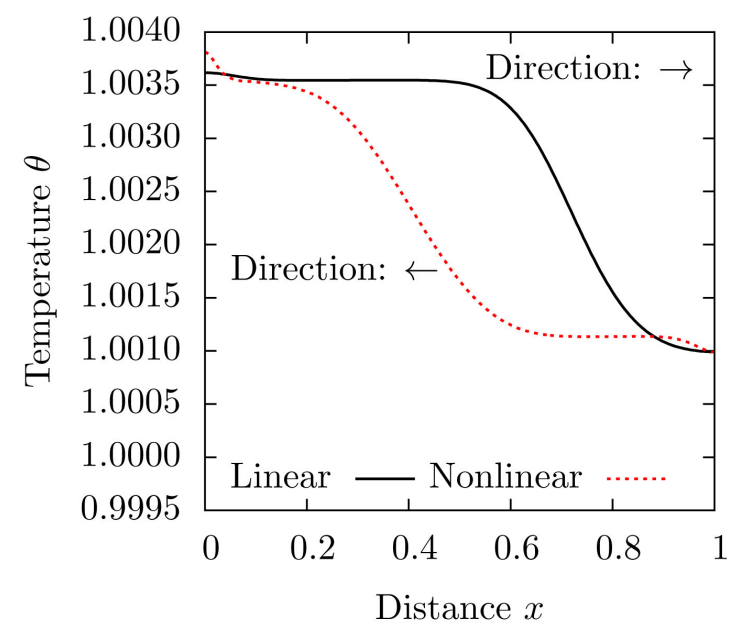

(f)

Figure 15. Evolution of temperature $\theta$ along the length of the rod: TVE, L2, $\Delta t=0.1, \sigma_{1}=0.4$. (a) $t=5 \Delta t$; (b) $t=9 \Delta t$; (c) Reflection, $t=11 \Delta t$; (d) Details of reflection, $t=11 \Delta t$; (e) $t=17 \Delta t$; (f) $t=23 \Delta t$. 
TVEM are solid continua with dissipation and memory (rheology). If the damping coefficient is same in TVE and TVEM, then the dissipation remains the same in both. Thus for the same damping coefficient in TVE and TVEM, the only difference in the behavior of stress wave in TVEM compared to TVE solid is due to rheology i.e. stress relaxation. Thus, in this study the most meaningful illustration is the comparison of nonlinear stress waves for TVE and TVEM. We choose $c=0.006$ in both TVE and TVEM and De $=0.002$ for TVEM. The studies are conducted for loading L1 with $\sigma_{1}= \pm 0.1$ i.e. a compressive and tensile pulse loading. Figures 16(a)-(f) show plots of the stress pulse propagation and reflection for TVE solid continua and TVEM for $\sigma_{1}=-0.1$ at times $t=5 \Delta t, 9 \Delta t, 11 \Delta t, 17 \Delta t$, and $23 \Delta t$.

Due to damping, the wave magnitudes progressively diminish along with base elongation as evolution proceeds. For TVEM, the peak values of pulse are consistently higher due to rheology, i.e. stress relaxation. In this case, the relaxation time $(D e)$ controls the relaxed state and hence additional time is required to achieve the same lower peak values as for TVE solid continua. For example, in Figure 16(a), Figure 16 (b), Figure 16 (e), and Figure 16(f), the peaks corresponding to TVEM (dashed line) will achieve the same lower values as the corresponding peaks for TVE solid continua (solid lines) if more time was allowed to elapse. Secondly, we note that the supports of the stress waves for TVEM are shorter than those of the corresponding TVE solid continua.

Similar results are presented in Figures 17(a)-(f) for $\sigma_{1}=0.1$ i.e. tensile wave. The behavior of the stress wave in TVE solid continua and TVEM is similar to what has been described for compressive stress wave.

\section{Loading L2: Tensile}

For loading L2, we consider $c=0.006, D e=0.001$ and $\sigma_{1}=0.4$ (tension). Computed evolution for linear and nonlinear cases are shown in Figures 18(a)-(f) for stress and Figures 19(a)-(f) for temperature. We observe behavior similar to L2 tensile loading for TVE Figures 14(a)-(f) and Figures 15(a)-(f). Steepening of nonlinear wave and formation of stress and temperature shocks is clearly observed in Figure 18(a) and Figure 18(b) and Figure 19(a) and Figure 19(b).

\subsubsection{Evolution for Large Values of Time: Tensile}

In the studies presented here, we consider loading L2 for TVE and also for TVEM. We choose $\sigma_{1}=0.4$ (tensile $\sigma_{x x}^{[0]}$ ), damping coefficient $c=0.006, \Delta t=0.1, k_{1}=k_{2}=2, p=9$ for the same descritiztion for a space-time strip as used in earlier studies for both TVE solid continua and TVEM. Evolution is computed for 4000 time steps i.e. 400 units of time that corresponds to 4.44 seconds as $t_{0}$ in this case is 0.0111 seconds.

Figure 20 shows plots of displacement $u$ at $x=1.0$ versus time $t$ for $0 \leq t \leq 400$ for TVE solid continua for $f=0$ (linear case) and $f=1$ (nonlinear case). Similar plots for linear and nonlinear cases for TVEM at $D e=0.001$ are shown in Figure 21. From Figure 20 and Figure 21, we observe that linear and nonlinear responses are drastically different for TVE as well as for TVEM in terms of peak negative and positive displacement values and mean values of displacements. The residual functional $I$ values for each space-time strip are $O\left(10^{-7}\right)$ or lower confirming the time accuracy of the evolution. A similar study for TE solid continua further confirms that the computations are almost free of numerical dispersion (as the peaks are maintained and the base does not elongate). Thus, the results reported for TVE solid continua and TVEM are free of numerical dispersion. Upon further evolution, the stationary states for TVE solid continua and TVEM evolution studies are obtained. The displacement values $\left(\left.{ }^{s} u\right|_{x=1.0}\right)$ corresponding to the stationary states are

TVE Solid Continua:

$$
\begin{gathered}
\left.{ }^{s} u\right|_{x=1.0}=0.3999 \quad \text { (linear) } \\
\left.{ }^{s} u\right|_{x=1.0}=0.3416 \quad \text { (nonlinear) }
\end{gathered}
$$

TVEM Solid Continua:

$$
\begin{gathered}
\left.{ }^{s} u\right|_{x=1.0}=0.3999 \quad \text { (linear) } \\
\left.{ }^{s} u\right|_{x=1.0}=0.3416 \quad \text { (nonlinear) }
\end{gathered}
$$

These values of displacements at $x=1.0$ are almost the same as the mean values of the displacements in 


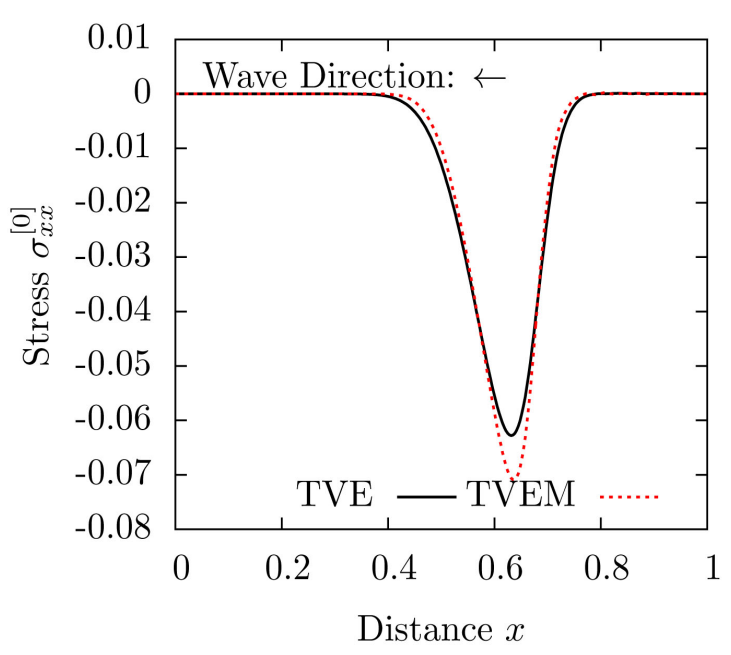

(a)

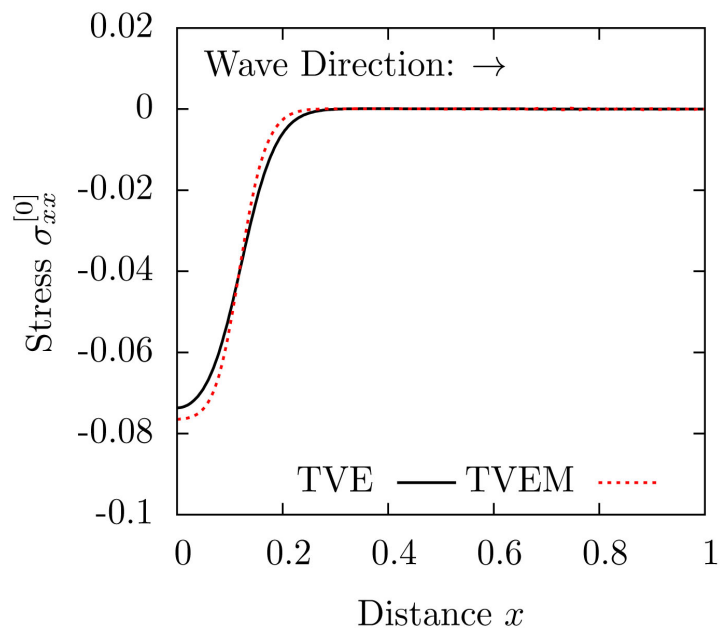

(c)

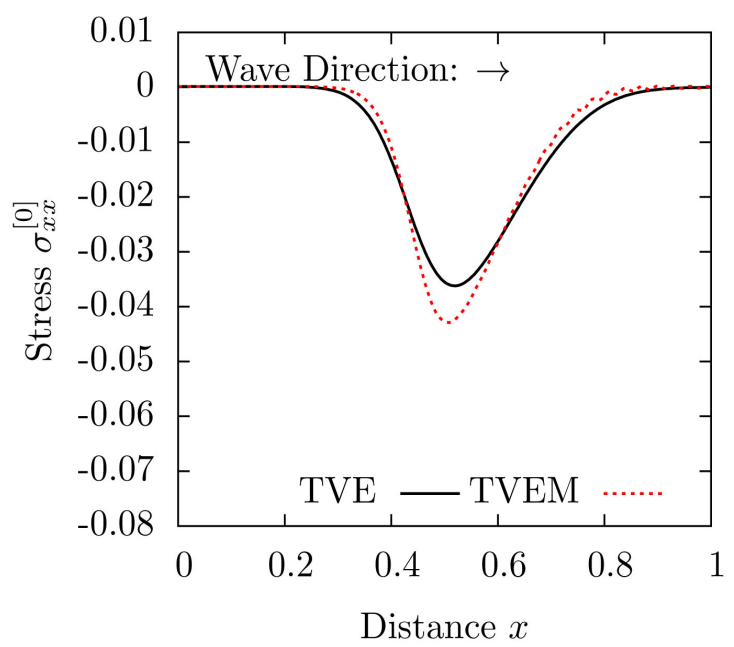

(e)

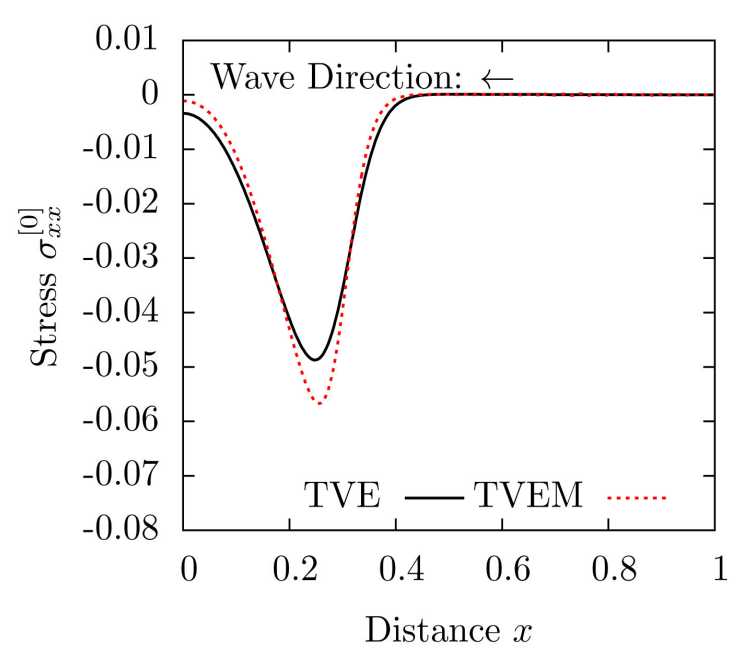

(b)

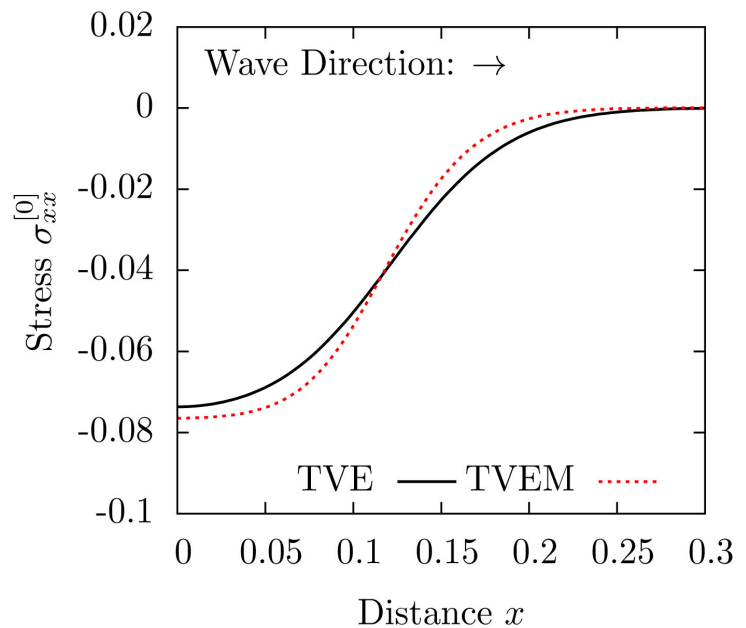

(d)

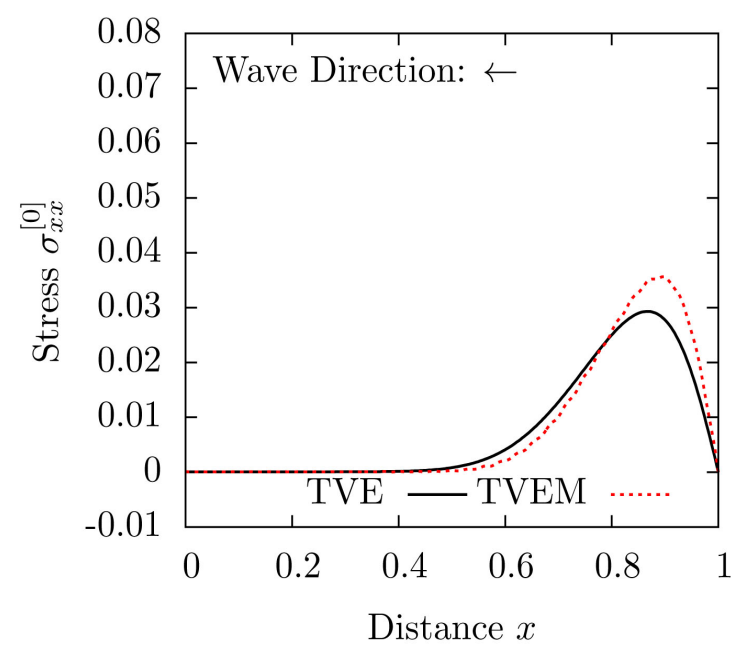

(f)

Figure 16. Comparison evolution of $\sigma_{x x}^{[0]}$ along the length of the rod: L1, $\Delta t=0.1, \sigma_{1}=-0.1$. (a) $t=5 \Delta t$; (b) $t=9 \Delta t$; (c) Reflection, $t=11 \Delta t$; (d) Details of reflection, $t=11 \Delta t$; (e) $t=17 \Delta t$; (f) $t=23 \Delta t$. 


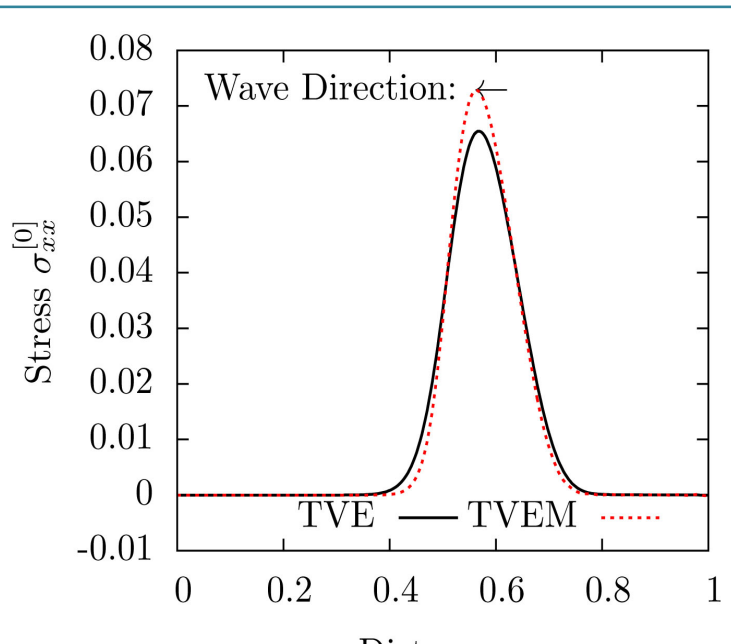

Distance $x$

(a)

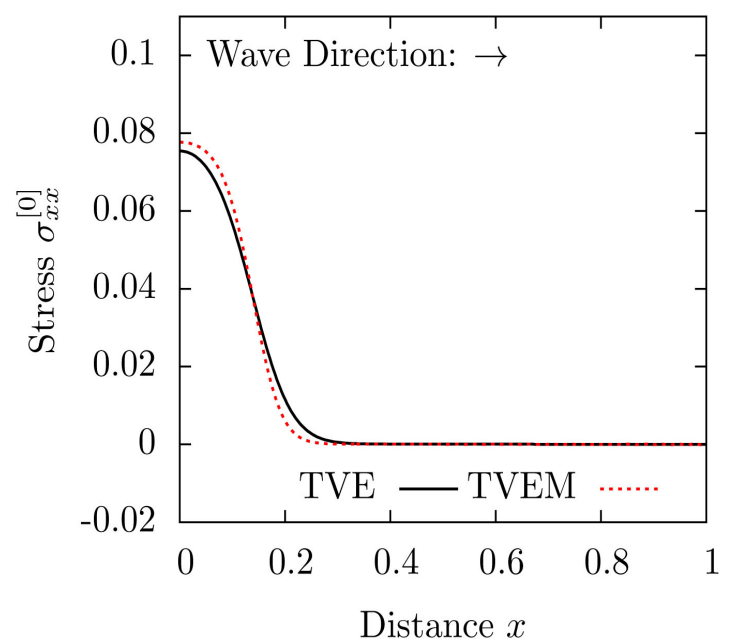

(c)

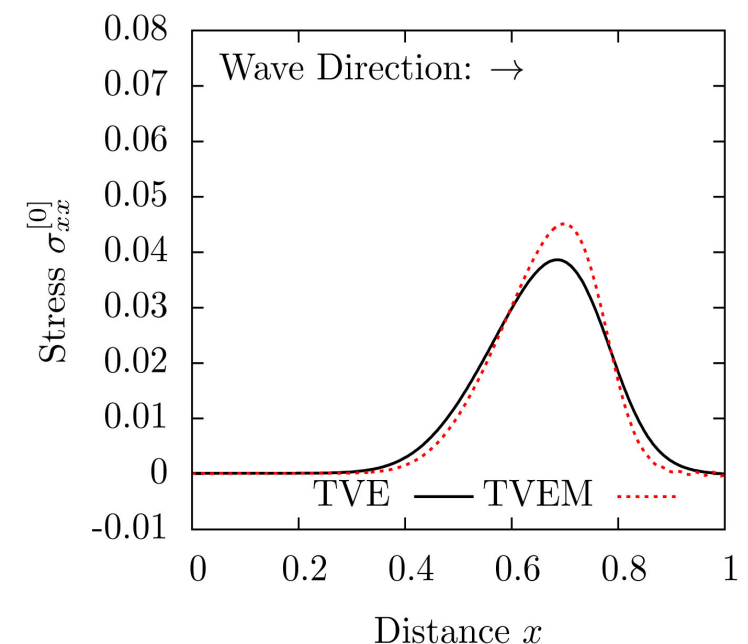

(e)

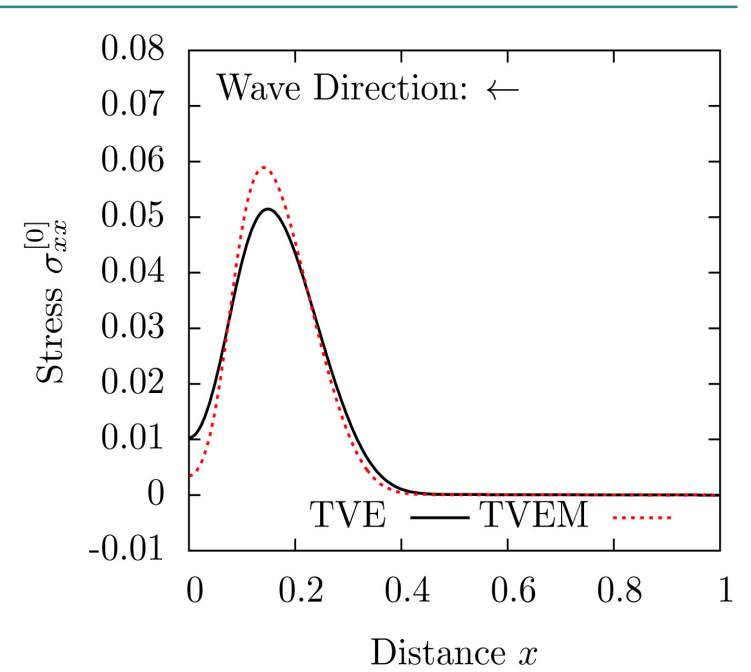

(b)

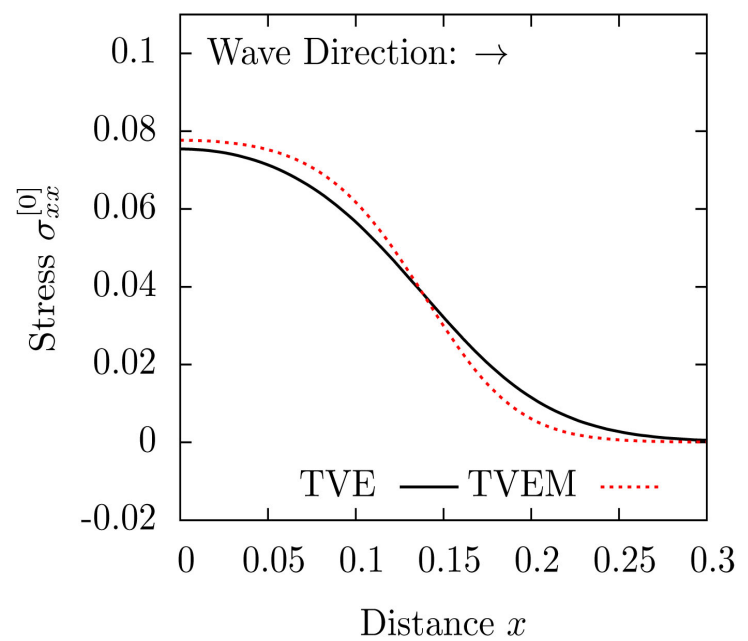

(d)

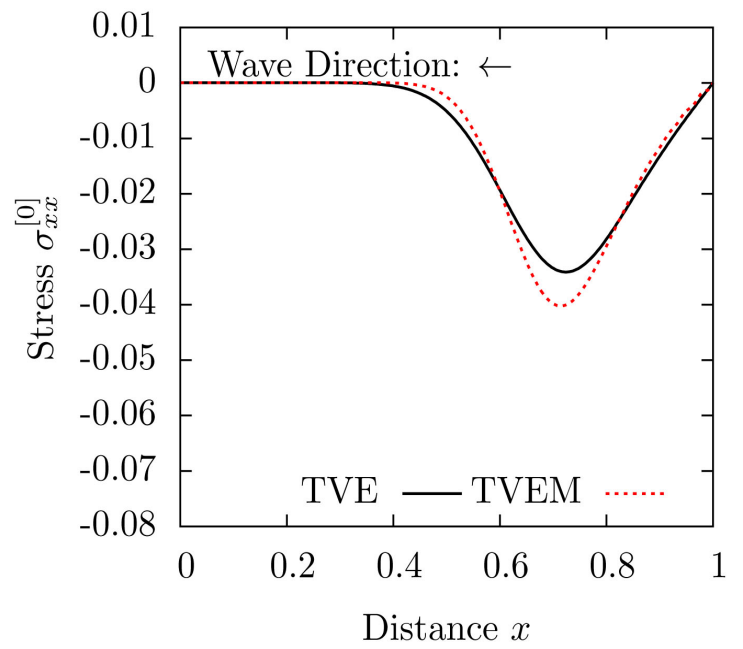

(f)

Figure 17. Comparison evolution of $\sigma_{x x}^{[0]}$ along the length of the rod: L1, $\Delta t=0.1, \sigma_{1}=0.1$. (a) $t=5 \Delta t$; (b) $t=9 \Delta t$; (c) Reflection, $t=11 \Delta t$; (d) Details of reflection, $t=11 \Delta t$; (e) $t=17 \Delta t$; (f) $t=23 \Delta t$. 


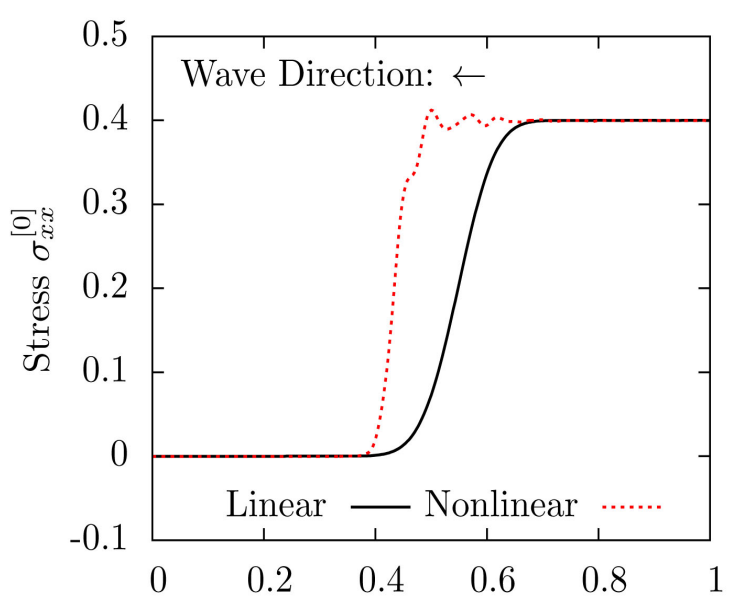

Distance $x$

(a)

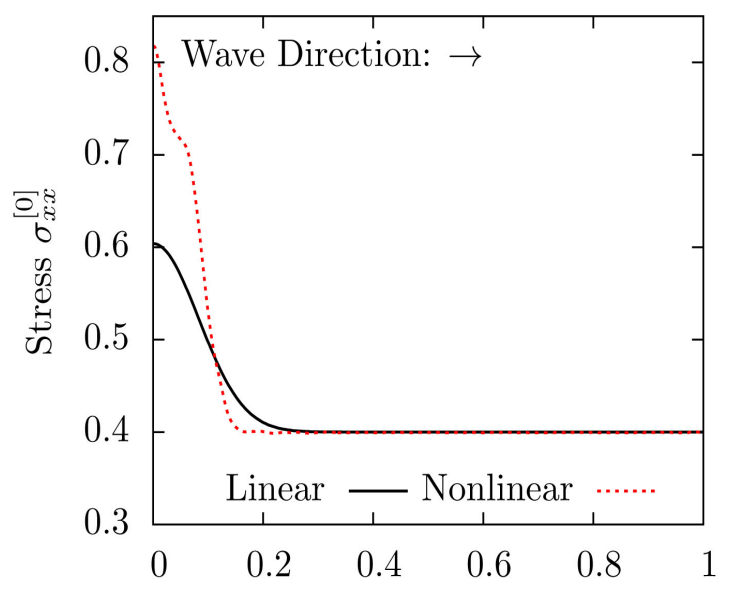

Distance $x$

(c)

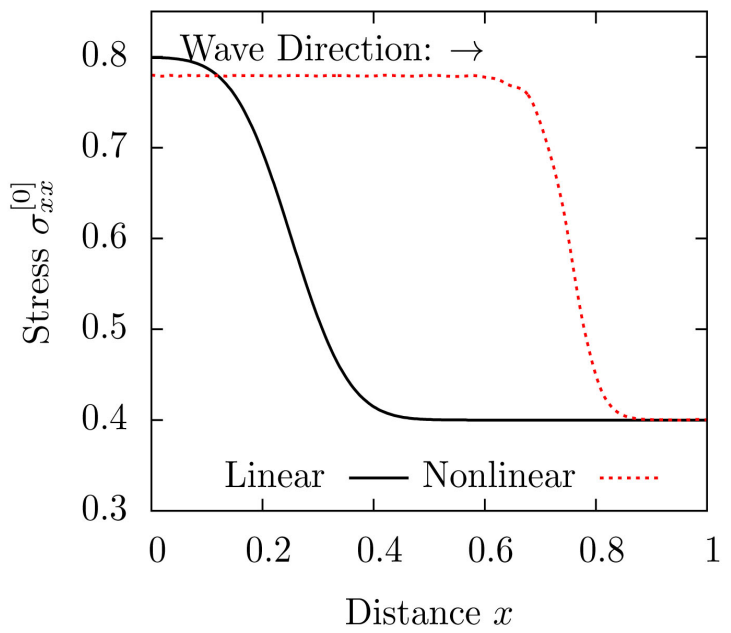

(e)

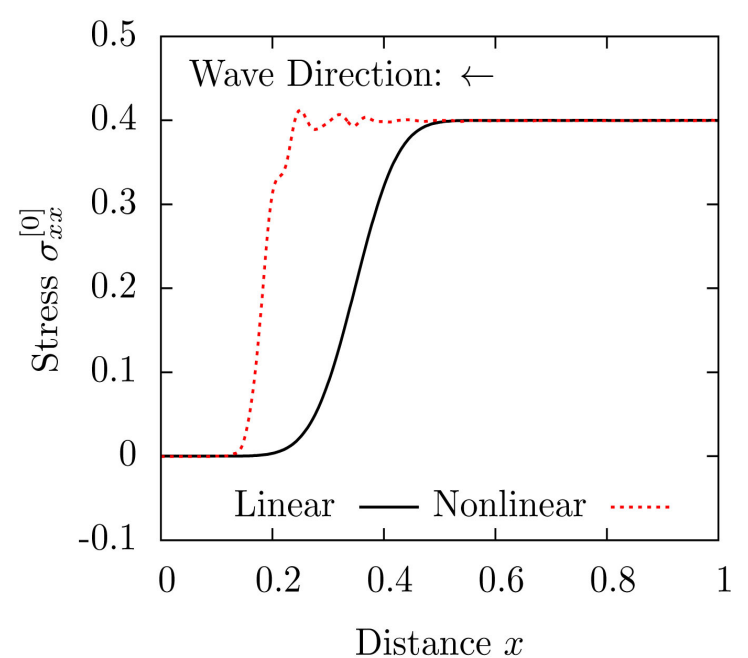

(b)

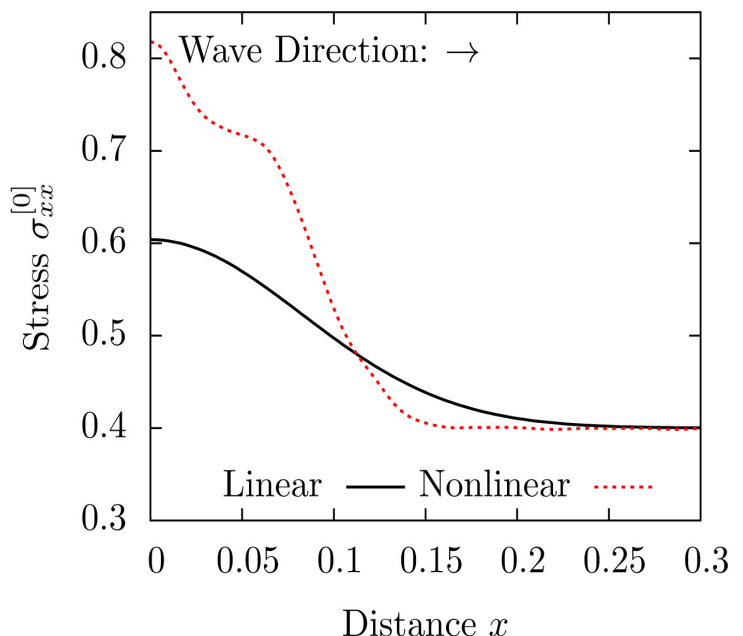

(d)

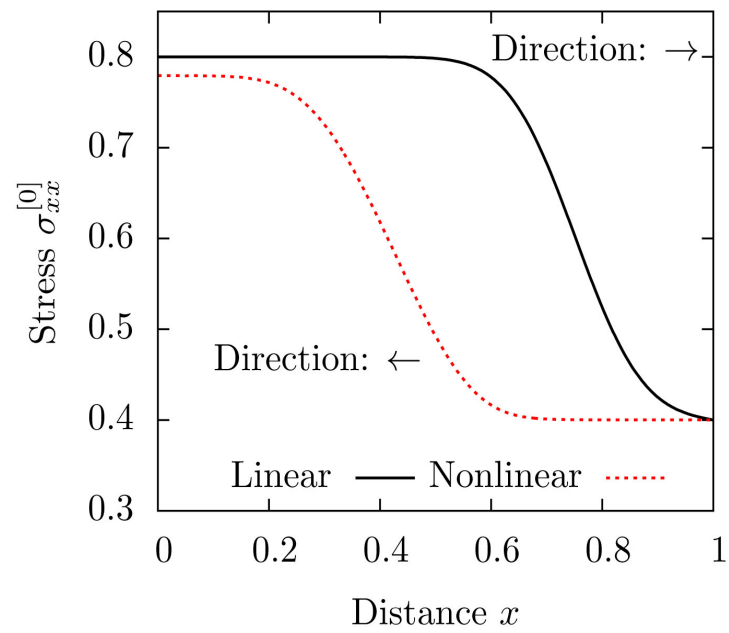

(f)

Figure 18. Evolution of $\sigma_{x x}^{[0]}$ along the length of the rod: TVEM, L2, $\Delta t=0.1, \sigma_{1}=0.4$. (a) $t=5 \Delta t$; (b) $t=9 \Delta t$; (c) Reflection, $t=11 \Delta t$; (d) Details of reflection, $t=11 \Delta t$; (e) $t=17 \Delta t$; (f) $t=23 \Delta t$. 


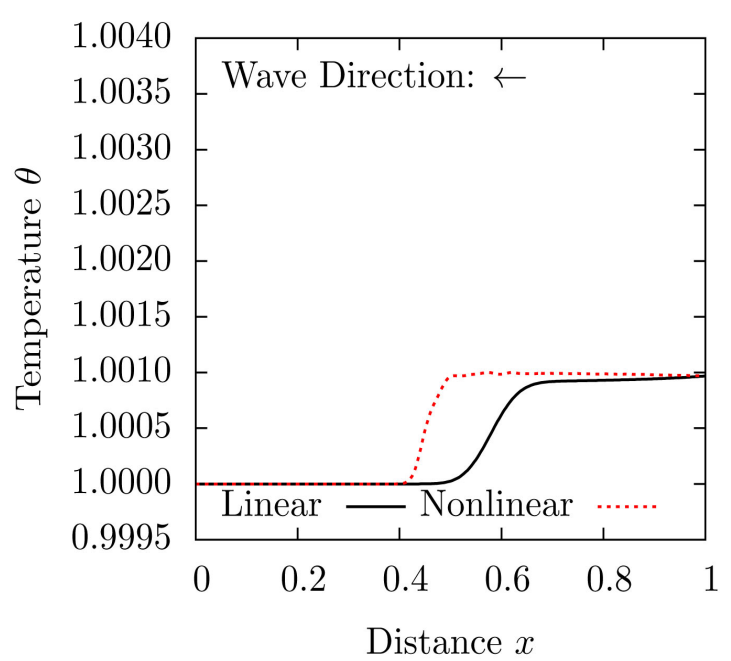

(a)

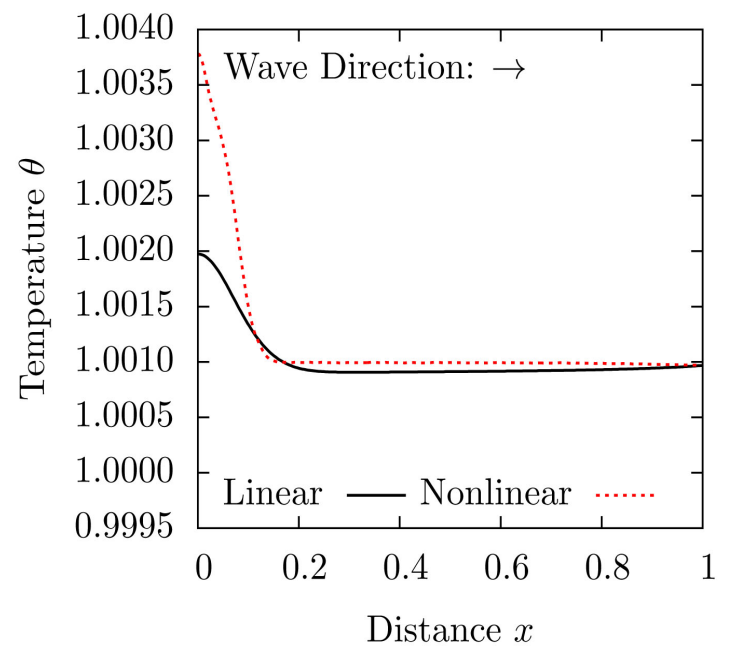

(c)

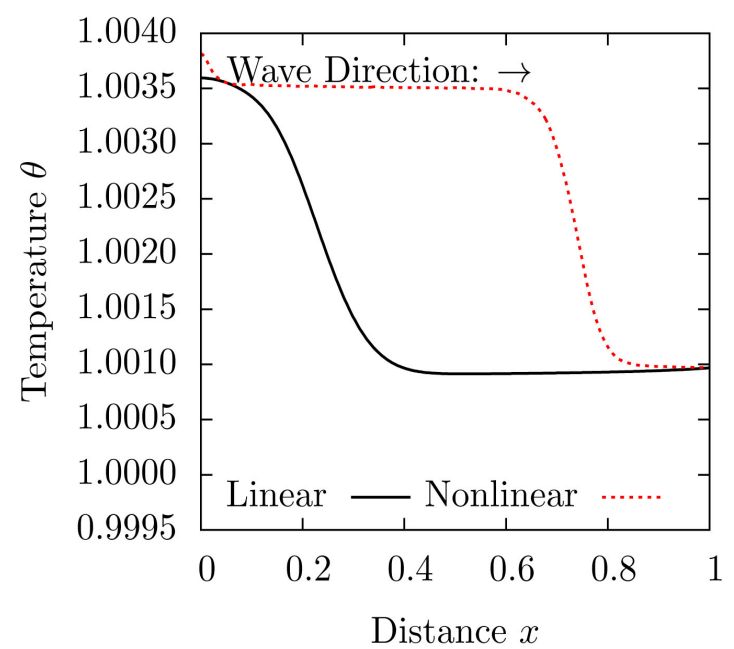

(e)

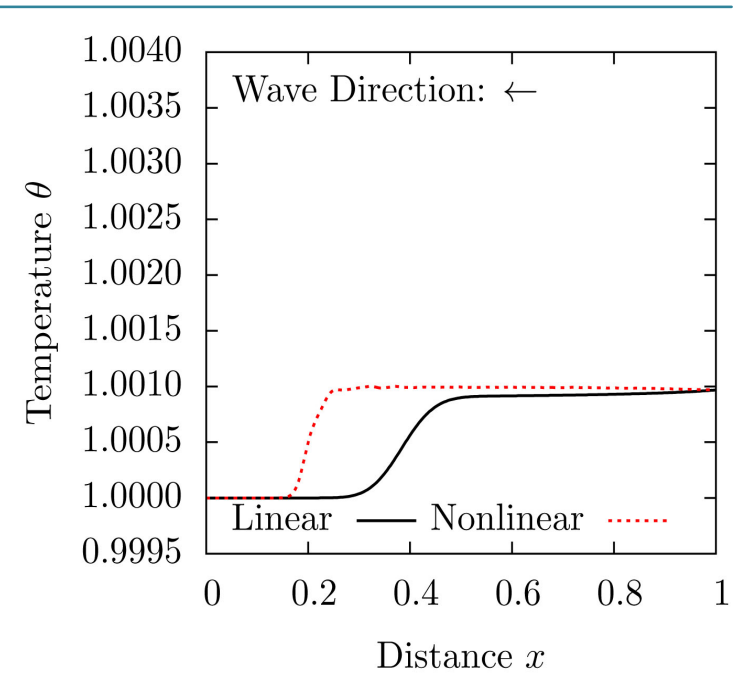

(b)

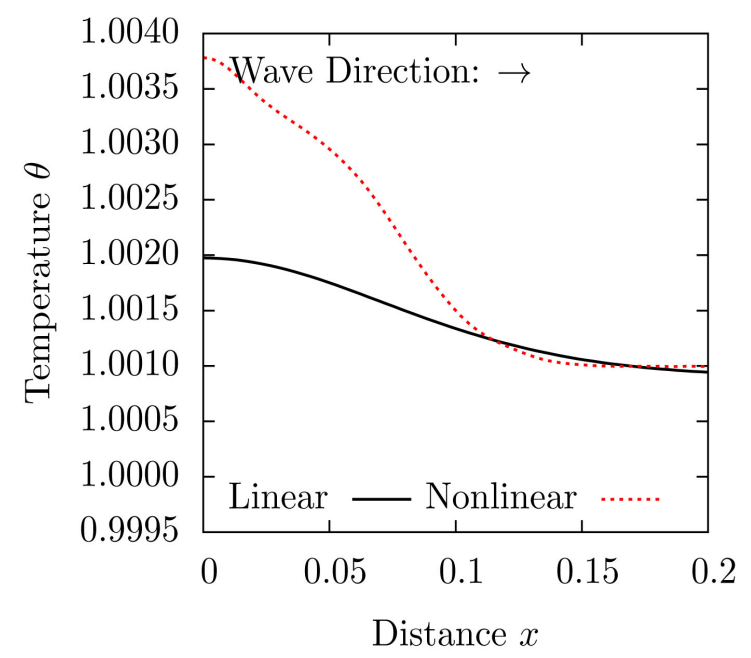

(d)

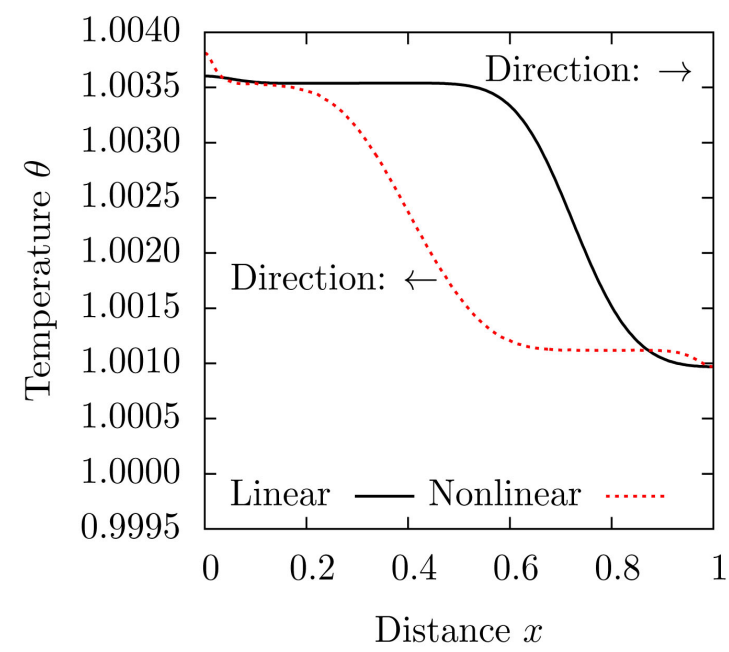

(f)

Figure 19. Evolution of temperature $\theta$ along the length of the rod: TVEM, L2, $\Delta t=0.1, \sigma_{1}=0.4$. (a) $t=5 \Delta t$; (b) $t=9 \Delta t$; (c) Reflection, $t=11 \Delta t$; (d) Details of reflection, $t=11 \Delta t$; (e) $t=17 \Delta t$; (f) $t=23 \Delta t$. 


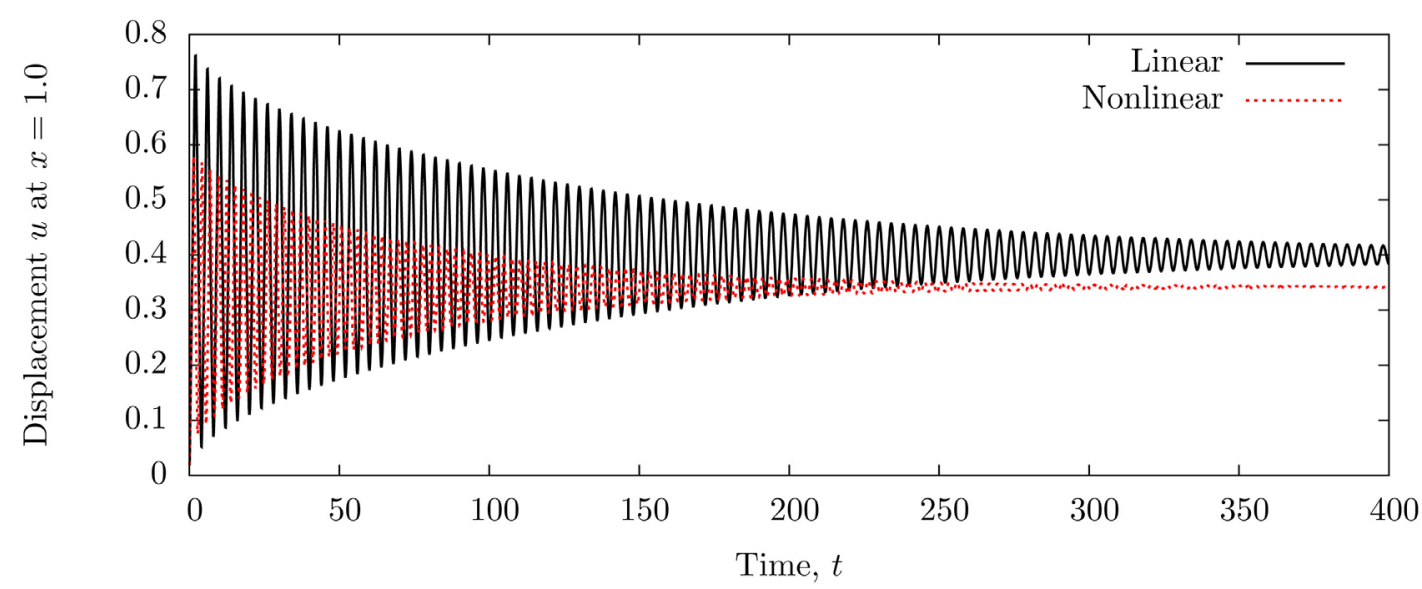

Figure 20. Displacement $\mu$ at $x=1: 0$ : TVE, L2, $\Delta t=0.1, \sigma_{1}=0.4$.

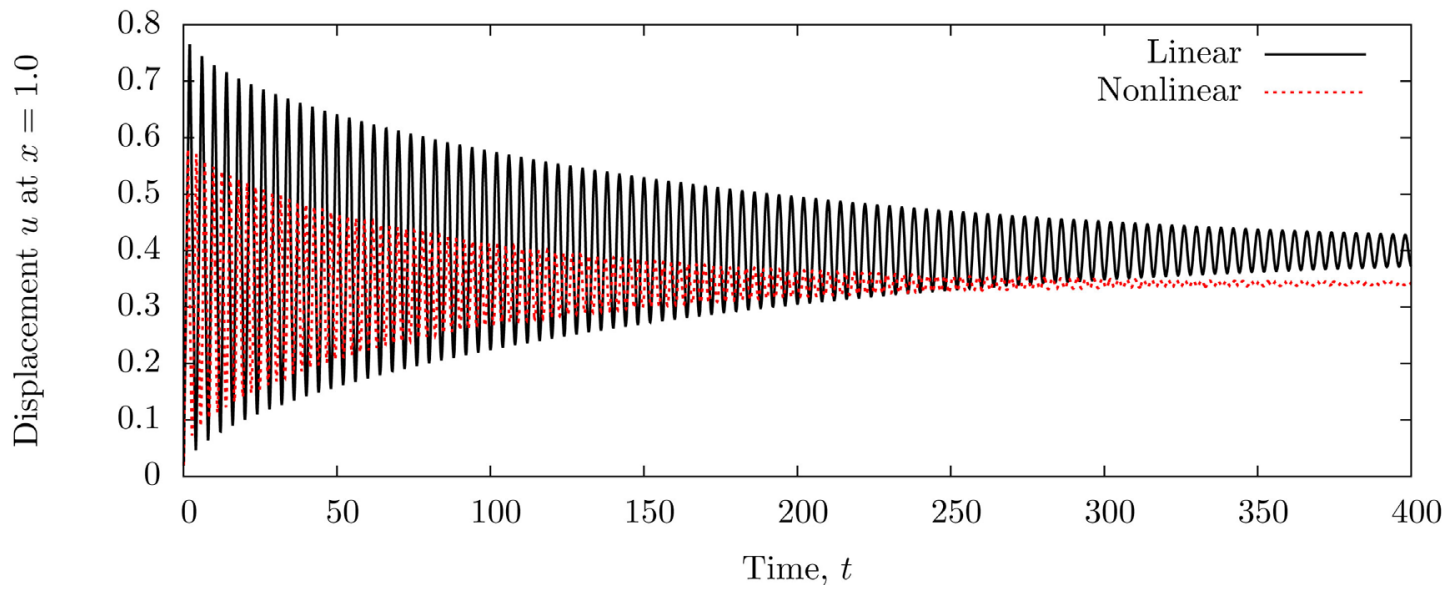

Figure 21. Displacement $\mu$ at $x=1: 0$ : TVEM, L2, $\Delta t=0.1, \sigma_{1}=0.4$.

Figure 20 and Figure 21. We observe that: 1) displacement $\left(\left.{ }^{s} u\right|_{x=1.0}\right)$ for the nonlinear case is lower than linear case as expected due to increase of stiffness caused by tensile stress field which results in lower values of displacement. This holds true in Figure 20 and Figure 21 as well during the evolution. 2) In the case of TVEM, the displacement values for $\left.{ }^{s} u\right|_{x=1.0}$ are exactly the same as those for TVE solid continua. This is due to the fact that upon complete stress relaxation the TVEM behavior is the same as the behavior of TVE solid continua. However, the peak values in Figure 21 for linear as well as nonlinear cases are not the same as the corresponding values in Figure 20. Figure 22 shows plots of peak positive displacement of the free end $\left(\left.u\right|_{x=1.0}\right)$ as a function of time $t$ for TVE solid continua and TVEM for both linear and nonlinear cases. The differences in the displacement values for TVE solid continua and TVEM solid continua for linear case $(f=0)$ are obviously due to rheology in TVEM. The same is true for TVE solid continua and TVEM for the nonlinear case. Drastically different values of displacements at $x=1.0$ for linear and nonlinear cases for both TVEM and TVE solid continua are quite obvious from Figure 22 as well as Figure 20 and Figure 21.

\section{Remarks}

Numerical studies were also conducted for loading L3 consisting of a velocity pulse. We note that if a velocity pulse of the same signature as generated by the loading L1 is applied at $x=1.0$, then the resulting stress pulse is the same as loading L1. Hence, the numerical solutions for the velocity pulse are intrinsically contained in the stress pulse loading. The value of Deborah number used here is quite small, hence the influence of rheology is not as pronounced as it would be for higher Deborah numbers. 


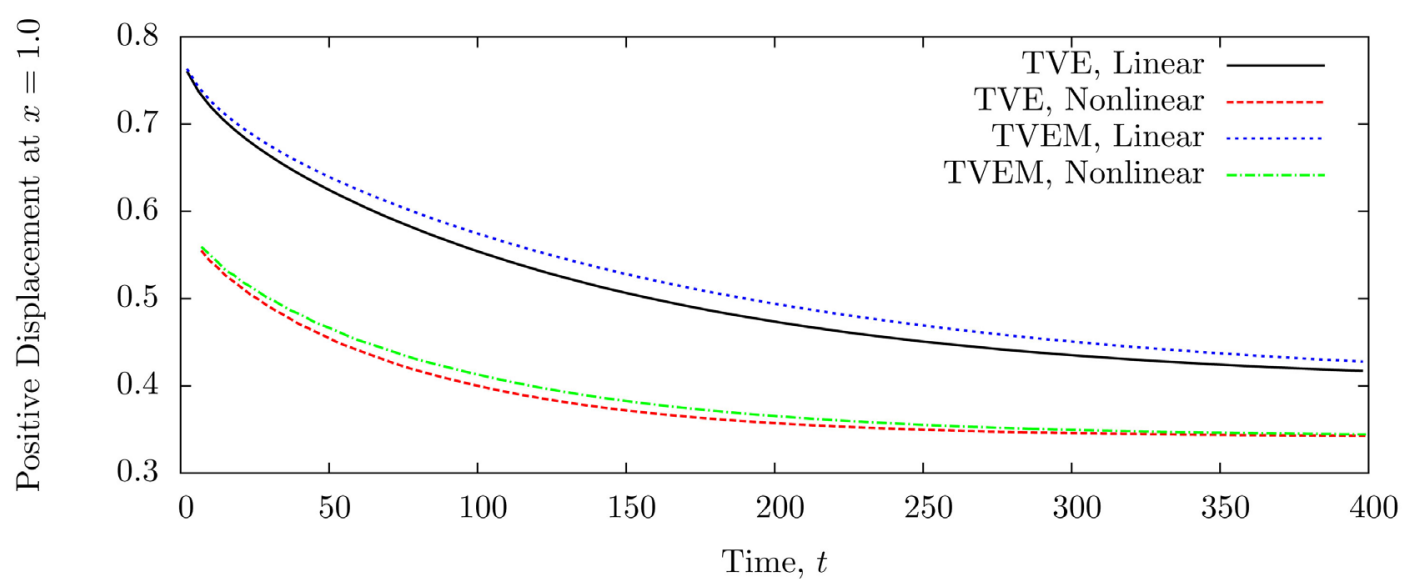

Figure 22. Peak positive displacement of free end $\left(\left.u\right|_{x=1.0}\right):$ TVE and TVEM, L2, $\Delta t=0.1, \sigma_{1}=0.4$.

\section{Summary and Conclusions}

In this paper, initiation, propagation, reflection, and the interaction of one-dimensional nonlinear waves in thermoelastic solid continua and thermoviscoelastic solid continua with and without memory have been presented. The mathematical models are first presented in $\mathrm{R}^{3}$ and then specialized for $\mathrm{R}^{1}$ for $1 \mathrm{D}$ wave propagation. The second Piola-Kirchoff stress and Green's strain tensors are used as conjugate pairs in the conservation and balance laws. The constitutive theory for the second Piola-Kirchoff stress tensor is a linear function of Green's strain tensor for TE. For TVE and TVEM, the constitutive theories are linear in strain tensor, its material derivative, and the material derivative of the second Piola-Kirchoff stress tensor. The constitutive theory used for heat vector is simple Fourier heat conduction law with constant thermal conductivity. The mathematical models for the nonlinear case consider the solid continua to be compressible. The mathematical models permit linear as well as nonlinear wave propagation studies. In the case of linear waves, the Green's strain tensor becomes linearized small strain tensor and the second Piola-Kirchoff stress tensor is simply Cauchy stress tensor. For linear wave propagation the solid matter is incompressible.

In the case of thermoelastic solid continua, the rate of mechanical work does not result in rate of entropy production, hence the energy equation can be decoupled from the rest of the mathematical model. In this case, deformation i.e. wave propagation and thermal effect can be studied separately. For thermoviscoelastic solid continua with and without memory, the rate of mechanical work results in entropy production; hence in these solid continua energy equation is integral part of the mathematical models. The present work is based on some assumptions in order to simplify the mathematical model.

1) The equilibrium second Piola-Kirchoff stress is expressed as a function of thermodynamic pressure (equation of state) and $[\mathrm{J}]$ is approximated by mean normal stress, thus avoiding equation of state altogether. This is an assumption, but in view of the fact that the main goal here is to study the nonlinearity in wave propagation due to Green's strain tensor, this assumption is not very crucial.

2) For compressible matter, the specific heat is a function of thermodynamic pressure $(p)$ and temperature or density and temperature due to $p=p(\rho, \theta)$. In the present work, a constant value of the specific heat is used.

3) Even though lack of precise account of compressibility in the energy equation may affect the overall results somewhat, the present forms used here are adequate enough to demonstrate the complex temperature distribution along the rod due to dissipation during wave propagation and reflection.

The space-time integral formulation based on space-time residual functional for a space-time strip with time marching is highly meritorious in (a) reducing the problem size, (b) ensuring accurate evolution for the current space-time strip before time marching is commenced. When the space-time residual functional is $O\left(10^{-6}\right)$ or lower only then time marching is commenced. This ensures time accurate evolution during the entire range of time. The orders of the scalar product approximation space in space and time $\left(k_{1}, k_{2}\right)$ are chosen to be 2 so that the space-time integrals over the discretization of the space-time strip are Riemann, an essential condition for time accurate evolution. 
From the numerical studies we observe the following.

1) In thermoelastic solid continua, linear or nonlinear waves maintain their amplitude and support for all space-time strips as well as for extended time evolution confirming that the computational process utilized here is elatively free of numerical dispersion.

2) The compressive nonlinear waves lag the linear waves due to increased density, hence reducing wave speed.

3) The tensile nonlinear waves lead the linear waves because of reduced density, hence increasing wave speed.

4) Both 2) and 3) hold for thermoelastic solid continua as well as thermoviscoelastic solid continua.

5) In both thermoviscoelastic solid continua with memory as well as the thermoviscoelastic solids without memory, the wave amplitude decays and the wave base elongates as evolution proceeds due to dissipation i.e. conversion of mechanical energy into entropy which results in temperature rise along the length of the rod. Complex temperature distribution due to dissipation is free of oscillations and is simulated without any difficulty together with the deformation field.

6) Progressively changing density due to compressibility or elongation results in progressively changing wave speed which finally results in piling up of waves forming a shock. This phenomenon exists in compressive as well as tensile nonlinear waves when the matter is compressible. Compared to linear waves, in the case of nonlinear compressive waves the shock formation occurs behind the linear wave, whereas in the case of tensile wave the shocks are formed ahead of the linear wave. Since in tension, large values of $\sigma_{1}$ can be used without occurrence of instability, the studies shown in Figures 18(a)-(f) for L2 loading with $\sigma_{1}=0.4$ clearly show the formation of shock wave ahead of the linear wave.

7) In the case of TVEM, the results are similar to TVE solid continua except that in case of TVEM momentarily higher stress magnitudes are observed during evolutions because of rheology.

8) From the extended time evolutions shown in Figure 20 and Figure 21 for TVE and TVEM (for L2 loading) for 4000 time steps we make some remarks.

a) Transient response has dramatically higher displacements than the static response. A rod of length one unit is elongated as much as 0.75 units during evolution.

b) Evolutions are smooth and free of numerical dispersion and are time accurate. This is confirmed by $I$ values $O\left(10^{-6}\right)$ or lower for each space-time strip.

c) Linear and nonlinear responses differ significantly. Tension increases the effective stiffness value as compression reduces it.

d) Peak positive displacements for linear and nonlinear cases for TVE and TVEM shown in Figure 22 show the differences in linear and nonlinear responses quite clearly.

This work demonstrates the significance of nonlinearity due to Green's strain and the need for incorporating it in wave propagation studies involving finite deformation. This is dramatically illustrated for tensile loading (L2) with $\sigma_{1}=0.4$. These studies presented here cannot be performed in a time accurate manner without using the mathematical models presented here and without using the space-time variationally consistent space-time finite element formulations, [21]-[30], based on space-time residual functional as used here. Extensions of this work for $\mathrm{R}^{2}$ as well as with the equation of state and with specific heat formulation that incorporates compressibility influence are currently in progress.

\section{Acknowledgements}

The computational infrastructure and the computational resources provided by the computational mechanics laboratory (CML) of the Department of Mechanical Engineering of the University of Kansas are gratefully acknowledged.

\section{References}

[1] Surana, K.S. (2014) Advanced Mechanics of Continua. CRC Press, Boca Raton.

[2] Engelbrecht, J. (1983) Nonlinear Wave Processes of Deformation in Solids. Pitman Publishing, London.

[3] Graham, R.A. (1993) Solids under High-Pressure Shock Compression. Springer-Verlag, New York. http://dx.doi.org/10.1007/978-1-4613-9278-1 
[4] Zarembo, L.K. and Krasil'nikov, V.A. (1970) Nonlinear Phenomena in the Propagation of Elastic Waves in Solids. Soviet Physics Uspekhi, 13, 778-797. http://dx.doi.org/10.1070/PU1971v013n06ABEH004281

[5] Fosdick, R., Ketema, Y. and Yu, J.H. (1997) A Non-linear Oscillator with History Dependent Force. International Journal of Non-Linear Mechanics, 33, 447-459. http://dx.doi.org/10.1070/PU1971v013n06ABEH004281

[6] Lima, W.J.N. de and Hamilton, M.F. (2003) Finite-Amplitude Waves in Isotropic Elastic Plates. Journal of Sound and Vibration, 265, 819-839. http://dx.doi.org/10.1016/S0022-460X(02)01260-9

[7] Gei, M., Bigoni, D. and Franceschini, G. (2004) Thermoelastic Small-Amplitude Wave Propagation in Nonlinear Elastic Multilayers. Mathematics and Mechanics of Solids, 9, 555-568. http://dx.doi.org/10.1177/1081286504038675

[8] Lima, W.J.N. de and Hamilton, M.F. (2005) Finite Amplitude Waves in Isotropic Elastic Waveguides with Arbitrary Constant Cross-Sectional Area. Wave Motion, 41, 1-11. http://dx.doi.org/10.1016/j.wavemoti.2004.05.004

[9] Renton, J.D. (1987) Applied Elasticity: Matrix and Tensor Analysis of Elastic Continua. Ellis Horwood, Chichester.

[10] Landau, L.D. and Lifshitz, E.M. (1986) Theory of Elasticity. Pergamon Press, New York.

[11] Engelbrecht, J., Berezovski, A. and Salupere, A. (2007) Nonlinear Deformation Waves in Solds and Dispersion. Wave Motion, 44, 493-500. http://dx.doi.org/10.1016/j.wavemoti.2007.02.006

[12] Shariyat, M., Lavasani, S.M.H. and Khaghani, M. (2010) Nonlinear Transient Thermal Stress and Elastic Wave Propagation Analyses of Thick Temperature-Dependent FGM Cylinders, Using a Second-Order Point-Collocation Method. Applied Mathematical Modeling, 34, 898-918. http://dx.doi.org/10.1016/j.apm.2009.07.007

[13] Yu, S.T.J., Yang, L., Lowe, R. and Bechtel, S.E. (2010) Numerical Simulation of Linear and Nonlinear Waves in Hypoelastic Solids by the CESE Method. Wave Motion, 47, 168-182. http://dx.doi.org/10.1016/j.wavemoti.2009.09.005

[14] Berezovski, A., Berezovski, M. and Engelbrecht, J. (2006) Numerical Simulation of Nonlinear Elastic Wave Propagation in Piecewise Homogeneous Media. Materials Science and Engineering A, 418, 364-369. http://dx.doi.org/10.1016/j.msea.2005.12.005

[15] Shariyat, M., Khaghani, M. and Lavasani, S.M.H. (2010) Nonlinear Thermoelasticity, Vibration, and Stress Wave Propagation Analyses of Thick FGM Cylinders with Temperature-Dependent Material Properties. European Journal of Mechanics A/Solids, 29, 378-391. http://dx.doi.org/10.1016/j.euromechsol.2009.10.007

[16] Li, Y., Vandewoestyne, B. and Abeele, K.V.D. (2012) A Nodal Discontinuous Galerkin Finite Element Method for Nonlinear Elastic Wave Propagation. Journal of the Acoustical Society of America, 131, 3650-3663. http://dx.doi.org/10.1121/1.3693654

[17] Shariyat, M. (2012) Nonlinear Transient Stress and Wave Propagation Analyses of the FGM Thick Cylinders, Employing a Unified Generalized Thermoelasticity Theory. International Journal of Mechanical Sciences, 65, 24-37. http://dx.doi.org/10.1016/j.ijmecsci.2012.09.001

[18] Yu, Y.M. and Lim, C.W. (2013) Nonlinear Constitutive Model for Axisymetric Bending of Annular Graphene-Like Nanoplate with Gradient Elasticity Enhancement Effects. Journal of Engineering Mechanics, 139, 1025-1035. http://dx.doi.org/10.1061/(ASCE)EM.1943-7889.0000625

[19] Nucera, C. and di Scalea, F.L. (2014) Nonlinear Semianalytical Finite-Element Algorithm for the Analysis of Internal Resonance Conditions in Complex Waveguides. Journal of Engineering Mechanics, 140, 502-522. http://dx.doi.org/10.1061/(ASCE)EM.1943-7889.0000670

[20] Surana, K.S., Maduri, R. and Reddy, J.N. (2006) One Dimensional Elastic Wave Propagation in Periodically Laminated Composites Using h; p; k Framework and STLS Finite Element Processes. Mechanics of Advanced Materials and Structures, 13, 161-196. http://dx.doi.org/10.1080/15376490500451809

[21] Surana, K.S. and Reddy, J.N. (2015) Mathematics of Computations and the Finite Element Method for Initial Value Problems. Book Manuscript in Progress.

[22] Surana, K.S., Ahmadi, A.R. and Reddy, J. (2002) The k-Version of Finite Element Method for Self-Adjoint Operators in BVP. International Journal of Computational Engineering Science, 3, 155-218. http://dx.doi.org/10.1142/S1465876302000605

[23] Surana, K.S., Ahmadi, A.R. and Reddy, J. (2003) The k-Version of Finite Element Method for Non-Self-Adjoint Operators in BVP. International Journal of Computational Engineering Science, 4, 737-812. http://dx.doi.org/10.1142/S1465876303002179

[24] Surana, K.S., Ahmadi, A.R. and Reddy J. (2004) The k-version of Finite Element Method for Nonlinear Operators in BVP. International Journal of Computational Engineering Science, 5, 133-207. http://dx.doi.org/10.1142/S1465876304002307

[25] Winterscheidt, D. and Surana, K.S. (1993) p-Version Least-Squares Finite Element Formulation for Convection-Diffusion Problems. International Journal for Numerical Methods in Engineering, 36, 111-133. http://dx.doi.org/10.1002/nme.1620360107 
[26] Winterscheidt, D. and Surana, K.S. (1994) p-Version Least Squares Finite Element Formulation for Two-Dimensional, Incopressible Fluid Flow. International Journal for Numerical Methods in Fluids, 18, 43-69. http://dx.doi.org/10.1002/fld.1650180104

[27] Bell, B.C. and Surana, K.S. (1994) A Space-Time Coupled p-Version Least-Squares Finite Element Formulation for Unsteady Fluid Dynamics Problems. International Journal for Numerical Methods in Engineering, 37, 3545-3569. http://dx.doi.org/10.1002/nme.1620372008

[28] Bell, B.C. and Surana, K.S. (1996) A Space-Time Coupled p-Version Least Squares Finite Element Formulation for Unsteady Two-Dimensional Navier-Stokes Equations. International Journal for Numerical Methods in Engineering, 39, 2593-2618. http://dx.doi.org/10.1002/(SICI)1097-0207(19960815)39:15<2593::AID-NME968>3.0.CO;2-2

[29] Surana, K.S., Reddy, J.N. and Allu, S. (2007) The k-Version of Finite Element Method for Initial Value Problems: Mathematical and Computational Framework. International Journal of Computational Methods in Engineering Science and Mechanics, 8, 123-136. http://dx.doi.org/10.1080/15502280701252321

[30] Surana, K.S., Allu, S., Reddy, J.N. and Tenpas, P.W. (2008) Least Squares Finite Element Processes in hpk Mathematical Framework for Non-Linear Conservation Law. International Journal of Numerical Methods in Fluids, 57, 1545-1568. http://dx.doi.org/10.1002/fld.1695

[31] Reddy, J.N. (2004) An Introduction to Nonlinear Finite Element Analysis. Oxford University Press, New York. http://dx.doi.org/10.1093/acprof:oso/9780198525295.001.0001

[32] Bathe, K.J. (1996) Finite Element Procedures. Prentice Hall, New Jersey.

[33] Riks, E. (1979) An Incremental Approach to the Solution of Snapping and Buckling Problems. International Journal of Solids and Structures, 15, 529-551. http://dx.doi.org/10.1016/0020-7683(79)90081-7 